\title{
Reductive Hydrazination with Trichlorosilane: A Method for the Preparation of 1,1-Disubstituted Hydrazines
}

\author{
Tao Wang, Xiao Di, Chao Wang, ${ }^{*}$ Li Zhou and Jian Sun* \\ Chengdu Institute of Biology, Chinese Academy of Sciences, Chengdu, 610041, China; Graduate School of Chinese \\ Academy of Sciences, Beijing, China
}

sunjian@cib.ac.cn; wangchao@cib.ac.cn

General: All starting materials were of the highest commercially available grade and used without further purification. All solvents used in the reactions were distilled from appropriate drying agents prior to use. Reactions were monitored by thin layer chromatography using silica gel HSGF254 plates. Flash chromatography was performed using silica gel HG/T2354-92. ${ }^{1} \mathrm{H}$ - and ${ }^{13} \mathrm{C}$ NMR (300, 400 or 600 and 75, 100 or $150 \mathrm{MHz}$, respectively) spectra were recorded in $\mathrm{CDCl}_{3}$. ${ }^{1} \mathrm{H} \mathrm{NMR}$ chemical shifts are reported in ppm (ס) relative to tetramethylsilane (TMS) with the solvent resonance employed as the internal standard $\left(\mathrm{CDCl}_{3}, \boldsymbol{\delta} 7.26 \mathrm{ppm}\right)$. Data are reported as follows: chemical shift, multiplicity $(\mathrm{s}=$ singlet, $\mathrm{d}=$ doublet, $\mathrm{t}=$ triplet, $\mathrm{q}=$ quartet, $\mathrm{m}=$ multiplet, $\mathrm{br}=$ broad, $\mathrm{dd}=$ double doublet), coupling constants $(\mathrm{Hz})$ and integration. ${ }^{13} \mathrm{C}$ NMR chemical shifts are reported in ppm from tetramethylsilane (TMS) with the solvent resonance as the internal standard $\left(\mathrm{CDCl}_{3}, \boldsymbol{\delta}\right.$ 77.0 ppm). ESIMS spectra were recorded on BioTOF Q. HPLC analyses were performed on PerkinElmer (Series 200 UV/VIS Detector and Series 200 Pump). Chiralpak OD-H and AD-H columns were purchased from Daicel Chemical Industries, LTD. All enantiomer ratios have been controlled by co-injections of the pure sample with the racemic substrates.

\section{General procedure for organocatalytic direct reductive hydrazination (for ketone).}

Under an argon atmosphere, the ketone $1(0.20 \mathrm{mmol})$ and hydrazine $2(0.20 \mathrm{mmol})$ and HMPA (3.5 $\mathrm{mg}, 0.02 \mathrm{mmol}$ ) were added in anhydrous DCM, stirred at room temperature for $10 \mathrm{~min}$, and then trichlorosilane ( $40 \mu \mathrm{L}, 0.40 \mathrm{mmol})$ was added. The reaction was quenched with saturated $\mathrm{NH}_{4} \mathrm{Cl}$ solution $(1 \mathrm{~mL})$, and then the $\mathrm{pH}$ was adjusted to $7 \sim 8$ with saturated $\mathrm{NaHCO}_{3}$. The mixture was extracted with EtOAc $(3 \times 15 \mathrm{~mL})$. The combined organic layer was washed with brine, dried over an- 
hydrous $\mathrm{MgSO}_{4}$, concentrated under reduced pressure and purified with column chromatography (silica gel, hexane/EtOAc) to affording pure hydrazine 3.

\section{1-phenyl-1-(1-phenylethyl)hydrazine (3a)}

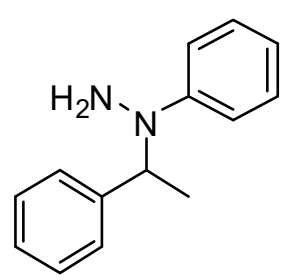

Colorless oil; $39 \mathrm{mg}$, Yield: $98 \%$; purified with flash chromatography (hexane/EtOAc $=20 / 1) ;{ }^{1} \mathbf{H}$ NMR $\left(300 \mathrm{MHz}, \mathrm{CDCl}_{3}\right): \boldsymbol{\delta} 7.35-7.29(\mathrm{~m}, 7 \mathrm{H}), 7.10(\mathrm{~d}$, $J=7.9 \mathrm{~Hz}, 2 \mathrm{H}), 6.82(\mathrm{t}, J=7.2 \mathrm{~Hz}, 1 \mathrm{H}), 5.15$ (q, $J=6.8 \mathrm{~Hz}, 1 \mathrm{H}), 3.12$ (brs, 2H), $1.57(\mathrm{~d}, J=6.8 \mathrm{~Hz}, 3 \mathrm{H}) ;{ }^{13} \mathbf{C} \mathbf{N M R}\left(75 \mathrm{MHz}, \mathrm{CDCl}_{3}\right): \boldsymbol{\delta} 151.3,140.9,129.0$, 128.4, 127.4, 127.2, 118.3, 114.0, 58.9, 14.6; HRMS (+ESI): $\mathrm{m} / \mathrm{z}$ calculated for $\left[\mathrm{C}_{14} \mathrm{H}_{16} \mathrm{~N}_{2}+\mathrm{H}\right]^{+}$ 213.1386, found 213.1396.

\section{1-(1-(4-fluorophenyl)ethyl)-1-phenylhydrazine (3b)}

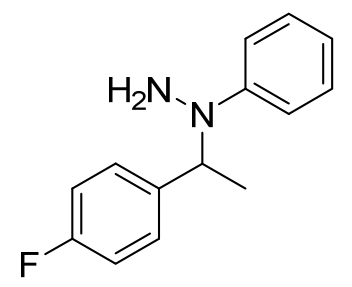

Colorless oil; $44 \mathrm{mg}$, Yield: $96 \%$; purified with flash chromatography (hexane/EtOAc $=20 / 1) ;{ }^{1} \mathbf{H}$ NMR $\left(600 \mathrm{MHz}, \mathrm{CDCl}_{3}\right): \boldsymbol{\delta} 7.24-7.17(\mathrm{~m}, 4 \mathrm{H}), 6.99$ $(\mathrm{d}, J=8.1 \mathrm{~Hz}, 2 \mathrm{H}), 6.92(\mathrm{t}, J=8.4 \mathrm{~Hz}, 2 \mathrm{H}), 6.73(\mathrm{t}, J=7.0 \mathrm{~Hz}, 1 \mathrm{H}), 5.00(\mathrm{q}, J$ $=7.0 \mathrm{~Hz}, 1 \mathrm{H}), 3.08$ (brs, 2H), 1.47 (d, $J=7.0 \mathrm{~Hz}, 3 \mathrm{H}) ;{ }^{13} \mathbf{C}$ NMR $(150 \mathrm{MHz}$, $\left.\mathrm{CDCl}_{3}\right): \delta 162.0(\mathrm{~d}, J=244.1 \mathrm{~Hz}), 151.1,136.8,129.1,129.0(\mathrm{~d}, J=6.8 \mathrm{~Hz}), 118.7,115.2(\mathrm{~d}, J=$ $21.7 \mathrm{~Hz}), 114.2,58.7,15.1$; HRMS (+ESI): $\mathrm{m} / \mathrm{z}$ calculated for $\left[\mathrm{C}_{14} \mathrm{H}_{15} \mathrm{FN}_{2}+\mathrm{H}\right]^{+} 231.1292$, found 231.1299 .

\section{1-(1-(4-chlorophenyl)ethyl)-1-phenylhydrazine (3c)}<smiles>CC(c1ccc(Cl)cc1)N(N)c1ccccc1</smiles>

Colorless oil; $46 \mathrm{mg}$, Yield: $93 \%$; purified with flash chromatography (hexane $/ E t O A c=20 / 1) ;{ }^{1} \mathbf{H}$ NMR $\left(300 \mathrm{MHz}, \mathrm{CDCl}_{3}\right): \boldsymbol{\delta} 7.29-7.25(\mathrm{~m}, 6 \mathrm{H}), 7.06$ (d, $J=8.0 \mathrm{~Hz}, 2 \mathrm{H}), 6.82(\mathrm{t}, J=7.3 \mathrm{~Hz}, 1 \mathrm{H}), 5.07$ (q, $J=6.8 \mathrm{~Hz}, 1 \mathrm{H}), 3.19$ (brs, 2H), $1.55(\mathrm{~d}, J=6.8 \mathrm{~Hz}, 3 \mathrm{H}) ;{ }^{13} \mathbf{C}$ NMR $\left(150 \mathrm{MHz}, \mathrm{CDCl}_{3}\right): \delta$ 151.2, 139.6, 132.8, 129.1, 128.7, 128.4, 118.6, 114.0, 58.5, 14.9; HRMS (+ESI) m/z calculated for $\left[\mathrm{C}_{14} \mathrm{H}_{15} \mathrm{ClN}_{2}+\mathrm{H}\right]^{+}$247.0997, found 247.0987.

\section{1-(1-(4-bromophenyl)ethyl)-1-phenylhydrazine (3d)}<smiles>CC(c1ccc(Br)cc1)N(N)c1ccccc1</smiles>

Colorless oil; $52 \mathrm{mg}$, Yield: $90 \%$; purified with flash chromatography (hexane/EtOAc $=20 / 1) ;{ }^{1} \mathbf{H}$ NMR $\left(300 \mathrm{MHz}, \mathrm{CDCl}_{3}\right): \boldsymbol{\delta} 7.29-7.19(\mathrm{~m}, 6 \mathrm{H}), 7.07$ (d, $J=8.1 \mathrm{~Hz}, 2 \mathrm{H}), 6.84(\mathrm{t}, J=7.4 \mathrm{~Hz}, 1 \mathrm{H}), 5.03$ (q, $J=6.8 \mathrm{~Hz}, 1 \mathrm{H}), 3.20$ (brs, 2H), $1.55(\mathrm{~d}, J=6.8 \mathrm{~Hz}, 3 \mathrm{H}) ;{ }^{13} \mathbf{C} \mathbf{N M R}\left(150 \mathrm{MHz}, \mathrm{CDCl}_{3}\right): \delta$ 151.3, 140.4, 
131.5, 129.2, 129.2, 121.1, 118.9, 114.2, 58.3, 15.1; HRMS (+ESI) m/z calculated for $\left[\mathrm{C}_{14} \mathrm{H}_{15} \mathrm{BrN}_{2}\right.$ $+\mathrm{H}]^{+}$291.0491, found 291.0491.

\section{1-phenyl-1-(1-(4-(trifluoromethyl)phenyl)ethyl)hydrazine (3e)}

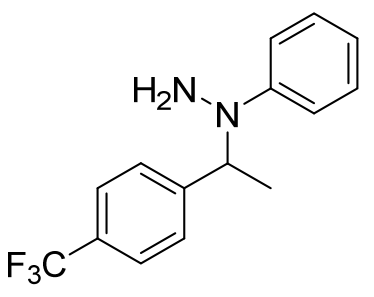

Colorless oil; $55 \mathrm{mg}$, Yield: $98 \%$; purified with flash chromatography (hexane/EtOAc $=20 / 1) ;{ }^{1} \mathbf{H}$ NMR $\left(300 \mathrm{MHz}, \mathrm{CDCl}_{3}\right): \boldsymbol{\delta} 7.60-7.46(\mathrm{~m}, 4 \mathrm{H})$, $7.27(\mathrm{q}, J=8.6 \mathrm{~Hz}, 2 \mathrm{H}), 7.06(\mathrm{~d}, J=8.0 \mathrm{~Hz}, 2 \mathrm{H}), 6.84(\mathrm{t}, J=7.2 \mathrm{~Hz}, 1 \mathrm{H}), 5.13$ $(\mathrm{q}, J=6.8 \mathrm{~Hz}, 1 \mathrm{H}), 3.20$ (brs, 2H), $1.60(\mathrm{~d}, J=6.8 \mathrm{~Hz}, 3 \mathrm{H}) ;{ }^{13} \mathbf{C}$ NMR (150 $\left.\mathrm{MHz}, \mathrm{CDCl}_{3}\right): \boldsymbol{\delta} 151.3,145.8,129.4(\mathrm{q}, J=32.1 \mathrm{~Hz}), 129.3,127.7,125.4(\mathrm{q}, J$ $=3.7 \mathrm{~Hz}), 124.2(\mathrm{q}, J=270.3 \mathrm{~Hz}), 118.9,113.9,58.9,15.3 ;$ HRMS (+ESI) m/z calculated for $\left[\mathrm{C}_{15} \mathrm{H}_{15} \mathrm{~F}_{3} \mathrm{~N}_{2}+\mathrm{H}\right]^{+}$281.1260, found 281.1256.

\section{1-(1-(4-nitrophenyl)ethyl)-1-phenylhydrazine (3f)}

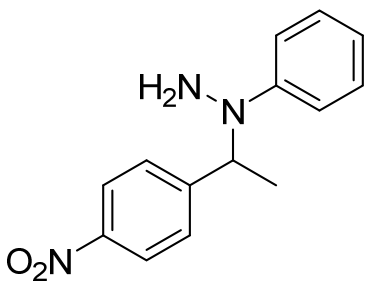

Red solid; $44 \mathrm{mg}$, Yield: $86 \%$; purified with flash chromatography (hexane/EtOAc $=6 / 1)$; m.p. $123-124{ }^{\circ} \mathrm{C} ;{ }^{1} \mathbf{H}$ NMR $\left(300 \mathrm{MHz}, \mathrm{CDCl}_{3}\right): \delta 8.18(\mathrm{~d}$, $J=7.0 \mathrm{~Hz}, 2 \mathrm{H}), 7.53(\mathrm{~d}, J=8.5 \mathrm{~Hz}, 2 \mathrm{H}), 7.32-7.26(\mathrm{~m}, 2 \mathrm{H}), 7.02(\mathrm{~d}, J=7.9$ Hz, 2H), 6.85 (t, $J=7.3 \mathrm{~Hz}, 1 \mathrm{H}), 5.13$ (q, $J=6.8 \mathrm{~Hz}, 1 \mathrm{H}), 3.31$ (brs, 2H), 1.62 $(\mathrm{d}, J=6.8 \mathrm{~Hz}, 3 \mathrm{H}) ;{ }^{13} \mathrm{C}$ NMR $\left(150 \mathrm{MHz}, \mathrm{CDCl}_{3}\right): \delta$ 151.1, 149.7, 147.1, 129.3, 128.2, 123.6, 120.0, 113.8, 59.0, 15.6; HRMS (+ESI) m/z calculated for $\left[\mathrm{C}_{14} \mathrm{H}_{15} \mathrm{~N}_{3} \mathrm{O}_{2}+\mathrm{H}\right]^{+} 258.1237$, found 258.1246.

\section{1-phenyl-1-(1-(p-tolyl)ethyl)hydrazine (3g)}

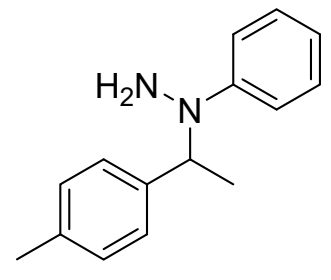

Colorless oil; $44 \mathrm{mg}$, Yield: $98 \%$; purified with flash chromatography (hexane/EtOAc $=20 / 1) ;{ }^{1} \mathbf{H}$ NMR $\left(300 \mathrm{MHz}, \mathrm{CDCl}_{3}\right): \boldsymbol{\delta} 7.29-7.20(\mathrm{~m}, 4 \mathrm{H}), 7.15-$ 7.09 (m, 4H), 6.80 (t, $J=7.3 \mathrm{~Hz}, 1 \mathrm{H}), 5.12$ (q, $J=6.8 \mathrm{~Hz}, 1 \mathrm{H}), 3.21$ (brs, 2H),

$2.34(\mathrm{~s}, 3 \mathrm{H}), 1.55(\mathrm{~d}, J=6.8 \mathrm{~Hz}, 3 \mathrm{H}) ;{ }^{13} \mathbf{C} \mathbf{N M R}\left(150 \mathrm{MHz}, \mathrm{CDCl}_{3}\right): \delta$ 151.6, 138.0, 136.9, 129.2, 129.1, 127.4, 118.4, 114.3, 59.0, 21.1, 14.8; HRMS (+ESI) m/z calculated for $\left[\mathrm{C}_{15} \mathrm{H}_{18} \mathrm{~N}_{2}+\mathrm{Na}\right]^{+} 249.1362$, found 249.1371 .

\section{1-(1-(4-methoxyphenyl)ethyl)-1-phenylhydrazine (3h)}<smiles>COc1ccc(C(C)N(N)C2=CCCC=C2)cc1</smiles>

Colorless oil; $41 \mathrm{mg}$, Yield: $84 \%$; purified with flash chromatography (hexane/EtOAc $=20 / 1) ;{ }^{1} \mathbf{H}$ NMR $\left(300 \mathrm{MHz}, \mathrm{CDCl}_{3}\right): \boldsymbol{\delta} 7.30-7.24(\mathrm{~m}, 4 \mathrm{H}), 7.05$ $(\mathrm{d}, J=7.9 \mathrm{~Hz}, 2 \mathrm{H}), 6.89-6.78(\mathrm{~d}, J=7.9 \mathrm{~Hz}, 2 \mathrm{H}), 5.11(\mathrm{q}, J=6.8 \mathrm{~Hz}, 1 \mathrm{H})$, 3.80 (s, 3H), 3.17 (brs, 2H), 1.54 (d, $J=6.8 \mathrm{~Hz}, 3 \mathrm{H}) ;{ }^{13} \mathrm{C}$ NMR (75 MHz, 
$\left.\mathrm{CDCl}_{3}\right): \delta 158.7,151.4,132.7,129.0,128.5,118.3,114.2,113.6,58.5,55.2,14.6 ;$ HRMS (+ESI) $\mathrm{m} / \mathrm{z}$ calculated for $\left[\mathrm{C}_{15} \mathrm{H}_{18} \mathrm{~N}_{2} \mathrm{O}+\mathrm{Na}\right]^{+} 265.1311$, found $\mathrm{m} / \mathrm{z} 265.1321$.

\section{1-(1-(naphthalen-1-yl)ethyl)-1-phenylhydrazine (3i)}

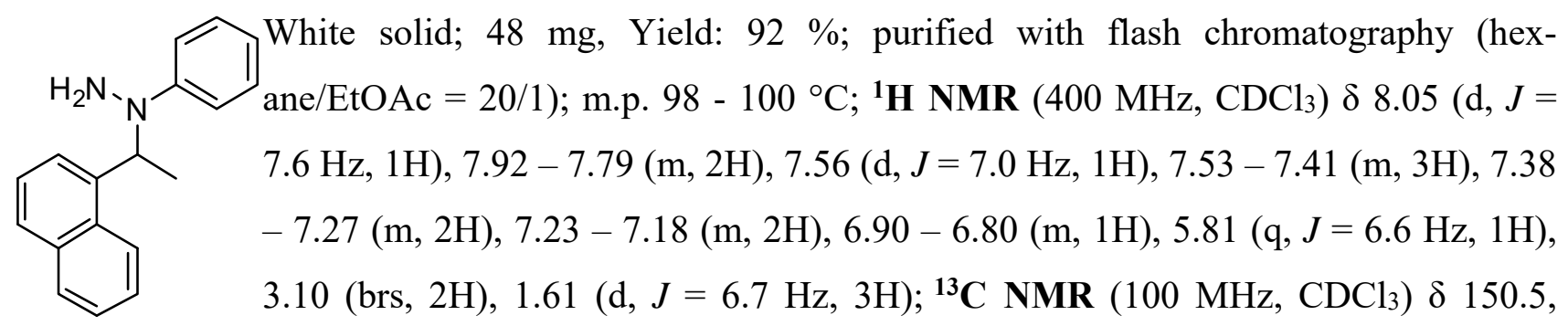
$136.8,133.9,131.9,129.4,128.7,128.4,126.5,125.8,125.1,125.0,123.8,117.9$, 113.2, 54.8, 12.2; HRMS (+ESI) m/z calculated for $\left[\mathrm{C}_{18} \mathrm{H}_{18} \mathrm{~N}_{2}+\mathrm{H}\right]^{+}$263.1543, found 263.1531.

\section{1-(1-(naphthalen-2-yl)ethyl)-1-phenylhydrazine (3j)}

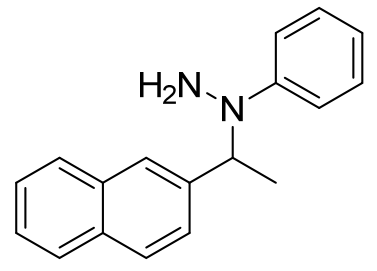

White solid; $49 \mathrm{mg}$, Yield: $94 \%$; purified with flash chromatography (hexane/EtOAc $=20 / 1)$; m.p. $107-109{ }^{\circ} \mathrm{C} ;{ }^{1} \mathbf{H}$ NMR $\left(300 \mathrm{MHz}, \mathrm{CDCl}_{3}\right): \boldsymbol{\delta} 7.84$ - $7.75(\mathrm{~m}, 4 \mathrm{H}), 7.51-7.46(\mathrm{~m}, 2 \mathrm{H}), 7.32-7.27(\mathrm{~m}, 3 \mathrm{H}), 7.15(\mathrm{~d}, J=8.0 \mathrm{~Hz}$, 2H), 6.83 (t, $J=7.2 \mathrm{~Hz}, 1 \mathrm{H}), 5.30$ (q, $J=6.8 \mathrm{~Hz}, 1 \mathrm{H}), 3.24$ (brs, 2H), 1.67 $(\mathrm{d}, J=6.8 \mathrm{~Hz}, 3 \mathrm{H}) ;{ }^{13} \mathbf{C}$ NMR $\left(150 \mathrm{MHz}, \mathrm{CDCl}_{3}\right): \delta$ 151.5, 138.8, 133.4, 132.8, 129.2, 128.2, 128.0, 127.6, 126.1, 125.9, 125.8, 118.5, 114.2, 59.3, 14.5; HRMS (+ESI) m/z calculated for $\left[\mathrm{C}_{18} \mathrm{H}_{18} \mathrm{~N}_{2}+\mathrm{H}\right]^{+} 263.1543$, found 263.1532 .

\section{1-phenyl-1-(1-phenylpropyl)hydrazine (3k)}

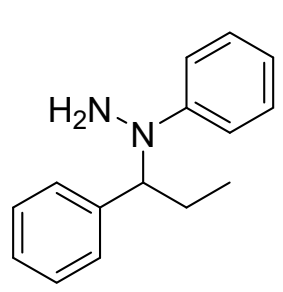

Colorless oil; $38 \mathrm{mg}$, Yield: $84 \%$; purified with flash chromatography (hexane/EtOAc $=20 / 1) ;{ }^{1} \mathbf{H}$ NMR $\left(300 \mathrm{MHz}, \mathrm{CDCl}_{3}\right): \delta 7.32-7.25(\mathrm{~m}, 7 \mathrm{H}), 7.04(\mathrm{~d}, J$ $=8.1 \mathrm{~Hz}, 2 \mathrm{H}), 6.77(\mathrm{t}, J=7.3 \mathrm{~Hz}, 1 \mathrm{H}), 4.79$ (q, $J=8.5 \mathrm{~Hz}, 1 \mathrm{H}), 3.20$ (brs, 2H), 2.21 - 2.07 (m, 1H), $2.05-1.98(\mathrm{~m}, 1 \mathrm{H}), 1.02$ (t, $J=7.3 \mathrm{~Hz}, 3 \mathrm{H}) ;{ }^{13} \mathbf{C}$ NMR (150 MHz, $\left.\mathrm{CDCl}_{3}\right): \delta 152.3,139.7,129.1,128.3,127.9,127.3,118.1,113.8,66.1,24.0,11.7$;

HRMS (+ESI) $\mathrm{m} / \mathrm{z}$ calculated for $\left[\mathrm{C}_{15} \mathrm{H}_{18} \mathrm{~N}_{2}+\mathrm{H}\right]^{+} 227.1543$, found 227.1543.

\section{1-phenyl-1-(1-phenylbutyl)hydrazine (3l)}

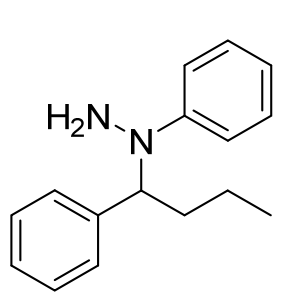

Colorless oil; $40 \mathrm{mg}$, Yield: $83 \%$; purified with flash chromatography (hexane/EtOAc $=20 / 1) ;{ }^{1} \mathbf{H}$ NMR $\left(600 \mathrm{MHz}, \mathrm{CDCl}_{3}\right): \delta 7.22-7.14(\mathrm{~m}, 7 \mathrm{H}), 6.95(\mathrm{~d}, J$ $=8.1 \mathrm{~Hz}, 2 \mathrm{H}), 6.68(\mathrm{t}, J=7.0 \mathrm{~Hz}, 1 \mathrm{H}), 4.82(\mathrm{q}, J=8.4 \mathrm{~Hz}, 1 \mathrm{H}), 3.12$ (brs, 2H), 
$2.11-2.05(\mathrm{~m}, 1 \mathrm{H}), 1.89-1.84(\mathrm{~m}, 1 \mathrm{H}), 1.42-1.22(\mathrm{~m}, 2 \mathrm{H}), 0.91(\mathrm{t}, J=7.3 \mathrm{~Hz}, 3 \mathrm{H}) ;{ }^{13} \mathbf{C} \mathbf{N M R}$ (150 MHz, $\left.\mathrm{CDCl}_{3}\right): \delta$ 152.1, 139.7, 129.1, 128.3, 127.9, 127.3, 118.1, 113.7, 64.0, 33.0, 20.2, 14.1; HRMS (+ESI) m/z calculated for $\left[\mathrm{C}_{16} \mathrm{H}_{20} \mathrm{~N}_{2}+\mathrm{H}\right]^{+} 241.1699$, found 241.1703 .

\section{1-(2-methyl-1-phenylpropyl)-1-phenylhydrazine (3m)}

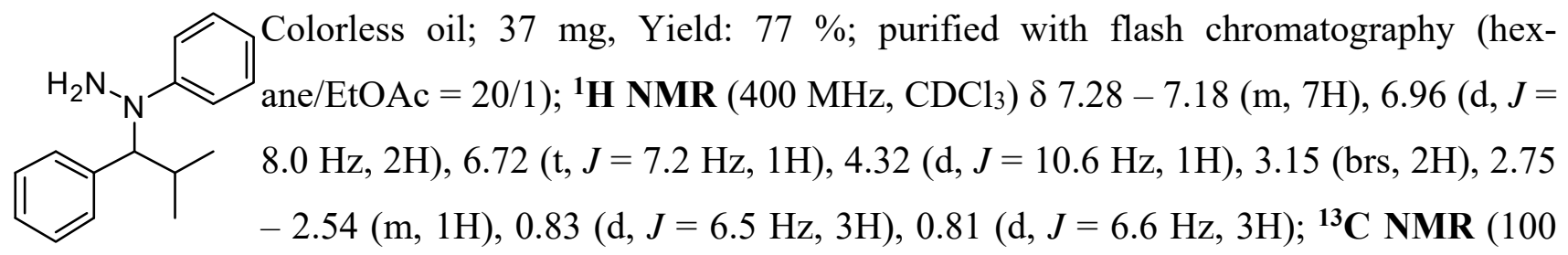
$\left.\mathrm{MHz}_{\mathrm{CDCl}}\right) \delta 152.2,138.0,129.0,128.5,128.2,127.3,118.0,113.7,72.2,28.7$,20.8, 20.4; HRMS $(+\mathrm{ESI}) \mathrm{m} / \mathrm{z}$ calculated for $\left[\mathrm{C}_{16} \mathrm{H}_{20} \mathrm{~N}_{2}+\mathrm{H}\right]^{+} 241.1699$, found 241.1688 .

\section{1-phenyl-1-(1-phenylpentyl)hydrazine (3n)}

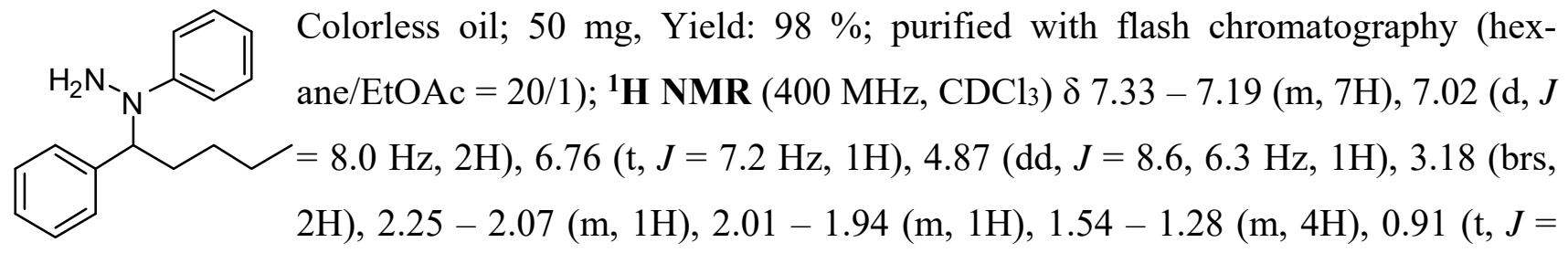

$6.9 \mathrm{~Hz}, 3 \mathrm{H}) ;{ }^{13} \mathbf{C}$ NMR $\left(100 \mathrm{MHz}, \mathrm{CDCl}_{3}\right) \delta 152.1,139.7,129.1,128.3,127.9,127.3,118.1,113.7$, 64.3, 30.6, 29.3, 22.8, 14.2. HRMS (+ESI) $\mathrm{m} / \mathrm{z}$ calculated for $\left[\mathrm{C}_{17} \mathrm{H}_{22} \mathrm{~N}_{2}+\mathrm{H}\right]^{+} 255.1856$, found 255.1849 .

\section{1-phenyl-1-(1-phenyloctyl)hydrazine (3o)}

$\begin{aligned} & \text { Colorless oil; } 44 \mathrm{mg} \text {, Yield: 75\%; purification by flash chromatography } \\ & (\text { hexane/EtOAc }=20 / 1) ;{ }^{1} \mathbf{H} \mathbf{N M R}\left(400 \mathrm{MHz}, \mathrm{CDCl}_{3}\right) \delta 7.32-7.18(\mathrm{~m},\end{aligned}$
$7 \mathrm{H}), 7.02(\mathrm{~d}, \mathrm{~J}=8.3 \mathrm{~Hz}, 2 \mathrm{H}), 6.75(\mathrm{t}, \mathrm{J}=7.2 \mathrm{~Hz}, 1 \mathrm{H}), 4.87(\mathrm{dd}, J=8.5$,
$6.3 \mathrm{~Hz}, 1 \mathrm{H}), 3.18$ (br.s., $2 \mathrm{H}), 2.22-2.08(\mathrm{~m}, 1 \mathrm{H}), 2.03-1.90(\mathrm{~m}, 1 \mathrm{H})$, $1.50-1.19(\mathrm{~m}, 10 \mathrm{H}), 0.87(\mathrm{t}, J=6.7 \mathrm{~Hz}, 3 \mathrm{H}) ;{ }^{13} \mathbf{C} \mathbf{N M R}\left(100 \mathrm{MHz}, \mathrm{CDCl}_{3}\right) \delta 152.1,139.7,129.1$, $128.3,127.9,127.3,118.1,113.7,64.3,31.9,30.9,29.7,29.3,27.0,22.7,14.2 ;$ HRMS (+ESI) m/z calculated for $\left[\mathrm{C}_{20} \mathrm{H}_{28} \mathrm{~N}_{2}+\mathrm{H}\right]^{+}$297.2325, found 297.2328.

\section{1-(cyclohexyl(phenyl)methyl)-1-phenylhydrazine (3p)}

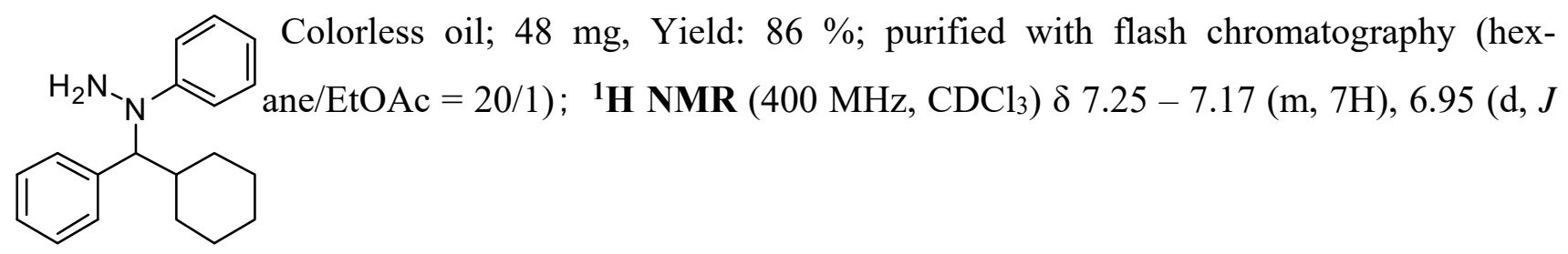


$=8.1 \mathrm{~Hz}, 2 \mathrm{H}), 6.71(\mathrm{t}, J=7.2 \mathrm{~Hz}, 1 \mathrm{H}), 4.44(\mathrm{~d}, J=10.6 \mathrm{~Hz}, 1 \mathrm{H}), 3.14$ (brs, 2H), $2.32(\mathrm{~m}, 1 \mathrm{H}), 2.05$ $(\mathrm{d}, J=13.0 \mathrm{~Hz}, 1 \mathrm{H}), 1.84-1.75(\mathrm{~m}, 1 \mathrm{H}), 1.73-1.60(\mathrm{~m}, 2 \mathrm{H}), 1.44(\mathrm{~d}, J=13.0 \mathrm{~Hz}, 1 \mathrm{H}), 1.36-1.31$ $(\mathrm{m}, 1 \mathrm{H}), 1.29-1.19(\mathrm{~m}, 2 \mathrm{H}), 1.17-1.08(\mathrm{~m}, 1 \mathrm{H}), 0.93-0.80(\mathrm{~m}, 1 \mathrm{H}) .{ }^{13} \mathbf{C} \mathbf{~ N M R}\left(100 \mathrm{MHz}, \mathrm{CDCl}_{3}\right)$ $\delta 152.3,137.8,129.0,128.7,128.2,127.3,117.9,113.6,70.6,37.9,31.1,30.8,26.8,26.2$. HRMS $(+\mathrm{ESI}) \mathrm{m} / \mathrm{z}$ calculated for $\left[\mathrm{C}_{19} \mathrm{H}_{24} \mathrm{~N}_{2}+\mathrm{H}\right]^{+} 281.2012$, found 281.2004.

\section{1-(cyclopropyl(phenyl)methyl)-1-phenylhydrazine (3q)}

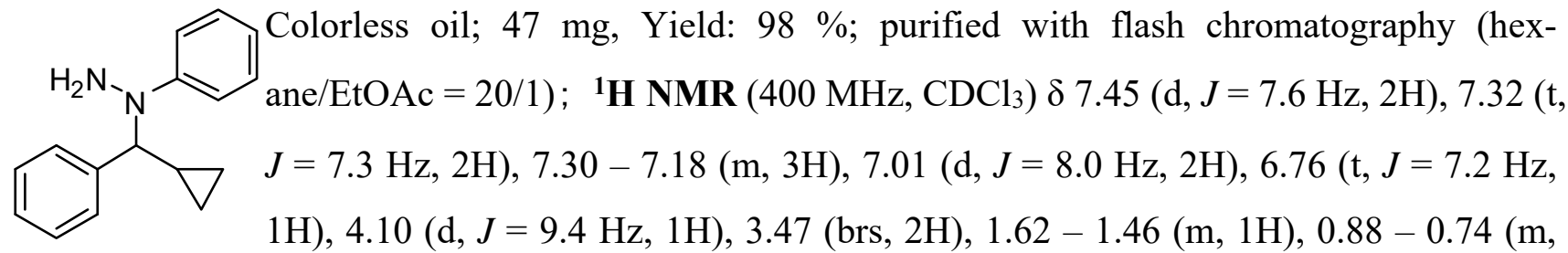
1H), $0.67-0.59(\mathrm{~m}, 1 \mathrm{H}), 0.51-0.31(\mathrm{~m}, 2 \mathrm{H}) .{ }^{13} \mathbf{C} \mathbf{N M R}\left(100 \mathrm{MHz}, \mathrm{CDCl}_{3}\right) \delta 152.2,140.7,129.1$, $128.5,127.5,127.3,118.0,113.4,70.4,13.0,5.6,4.0$. HRMS $(+\mathrm{ESI}) \mathrm{m} / \mathrm{z}$ calculated for $\left[\mathrm{C}_{16} \mathrm{H}_{18} \mathrm{~N}_{2}+\right.$ $\mathrm{H}]^{+}$239.1543, found 239.1534.

\section{1-phenyl-1-(1,2,3,4-tetrahydronaphthalen-1-yl)hydrazine (3r)}

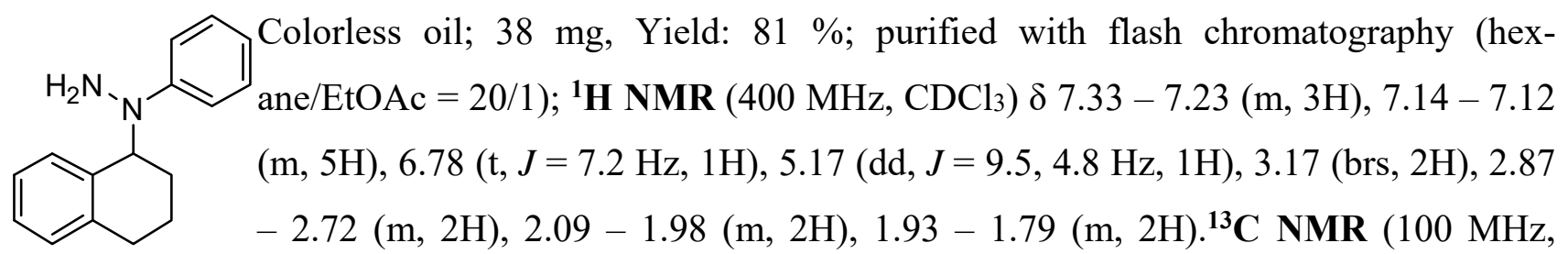
$\left.\mathrm{CDCl}_{3}\right) \delta 151.8,138.9,136.2,129.6,129.3,127.0,126.9,126.4,117.8,113.2,59.9,29.7,24.4,22.2$. HRMS (+ESI) m/z calculated for $\left[\mathrm{C}_{16} \mathrm{H}_{18} \mathrm{~N}_{2}+\mathrm{H}\right]^{+} 239.1543$, found 239.1533 .

\section{1-(2,3-dihydro-1H-inden-1-yl)-1-phenylhydrazine (3s)}

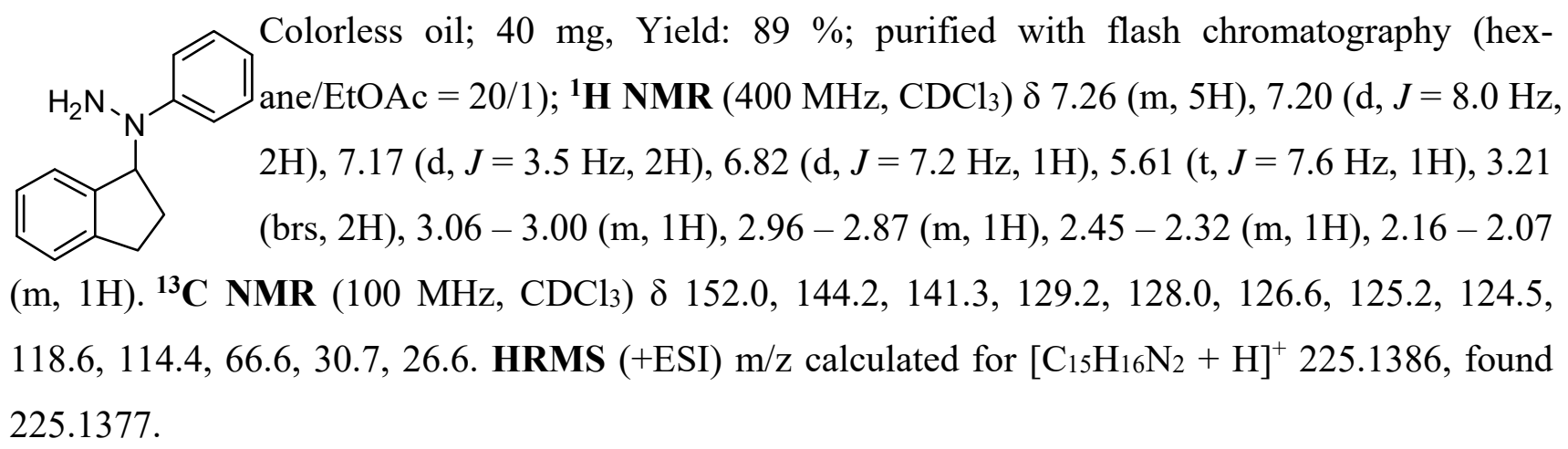

\section{1-benzhydryl-1-phenylhydrazine (3t)}




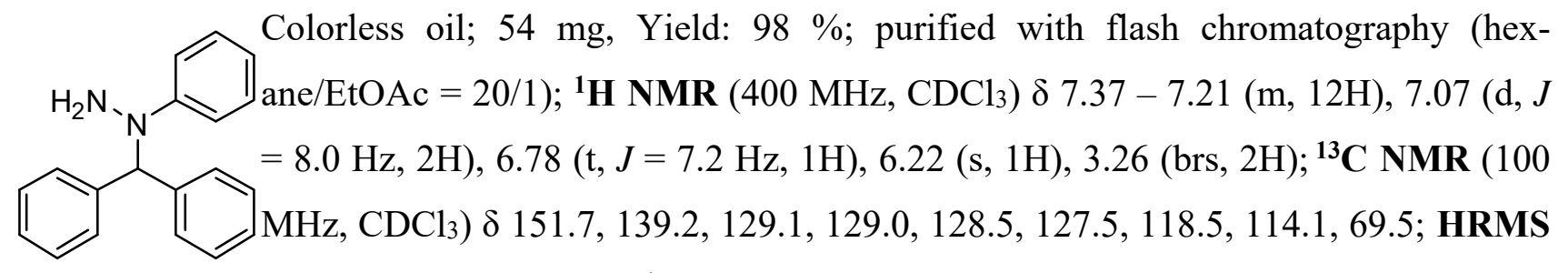
$(+\mathrm{ESI}) \mathrm{m} / \mathrm{z}$ calculated for $\left[\mathrm{C}_{19} \mathrm{H}_{18} \mathrm{~N}_{2}+\mathrm{H}\right]^{+} 275.1543$, found 275.1536 .

\section{1-(bis(4-fluorophenyl)methyl)-1-phenylhydrazine (3u)}<smiles>NN(c1ccccc1)C(c1ccc(F)cc1)c1ccc(F)cc1</smiles>

Colorless oil; $61 \mathrm{mg}$, Yield: $98 \%$; purified with flash chromatography (hexane/EtOAc $=20 / 1) ;{ }^{1} \mathbf{H}$ NMR $\left(400 \mathrm{MHz}, \mathrm{CDCl}_{3}\right) \delta 7.25-7.13(\mathrm{~m}, 6 \mathrm{H}), 7.06$ - $6.96(\mathrm{~m}, 6 \mathrm{H}), 6.81(\mathrm{t}, J=7.3 \mathrm{~Hz}, 1 \mathrm{H}), 6.12(\mathrm{~s}, 1 \mathrm{H}), 3.23$ (brs, 2H); ${ }^{13} \mathrm{C}$ NMR $\left(100 \mathrm{MHz}, \mathrm{CDCl}_{3}\right) \delta 162.2(\mathrm{~d}, J=244.8 \mathrm{~Hz}), 151.5,135.0(\mathrm{~d}, J=3.2$ $\mathrm{Hz}), 130.5$ (d, $J=7.9 \mathrm{~Hz}), 129.2,119.1,115.4$ (d, $J=21.2 \mathrm{~Hz}), 114.3$, 68.5. HRMS (+ESI) $\mathrm{m} / \mathrm{z}$ calculated for $\left[\mathrm{C}_{19} \mathrm{H}_{16} \mathrm{~F}_{2} \mathrm{~N}_{2}+\mathrm{H}\right]^{+} 311.1354$, found 311.1352 .

\section{1-((4-chlorophenyl)(phenyl)methyl)-1-phenylhydrazine (3v)}

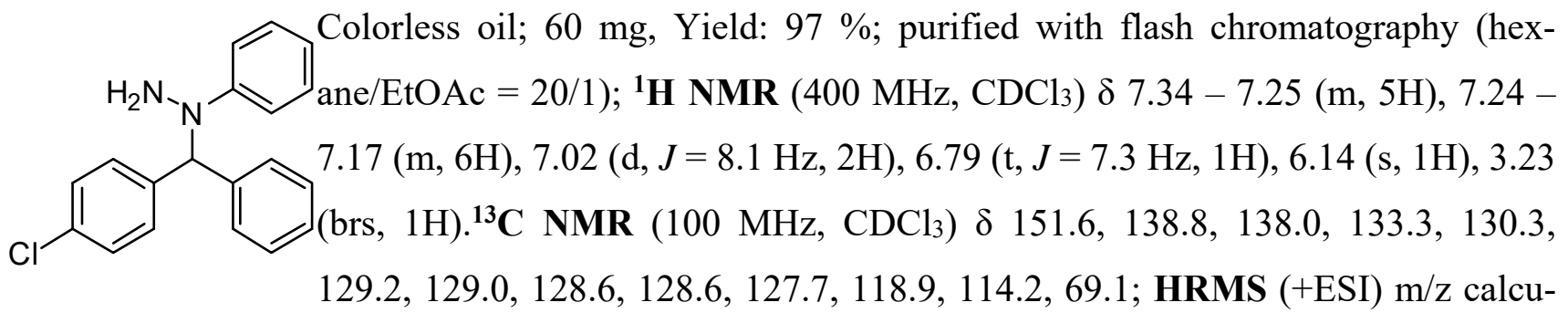
lated for $\left[\mathrm{C}_{19} \mathrm{H}_{17} \mathrm{ClN}_{2}+\mathrm{H}\right]^{+}$309.1153, found 309.1144.

\section{1-(1-cyclohexylethyl)-1-phenylhydrazine (3w)}

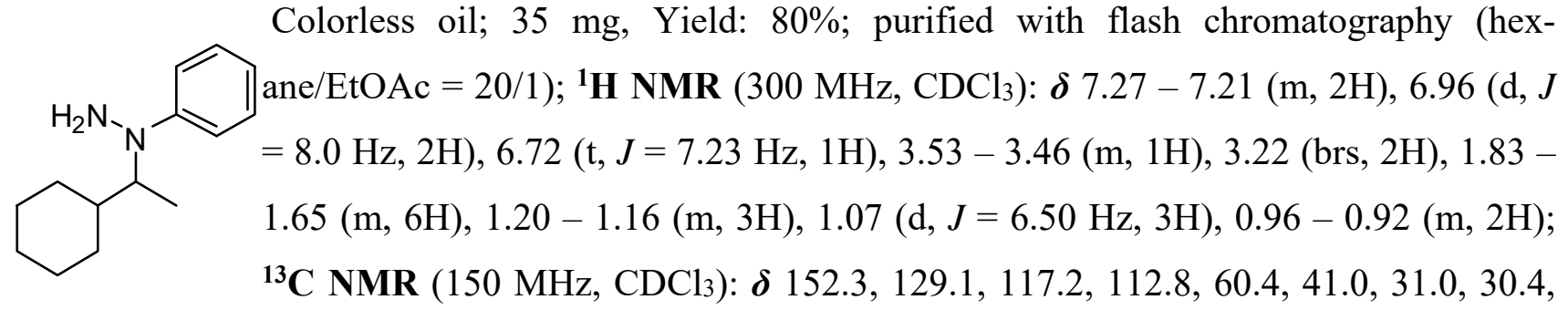

26.6, 26.4, 26.2, 12.5; HRMS (+ESI) m/z calculated for $\left[\mathrm{C}_{14} \mathrm{H}_{22} \mathrm{~N}_{2}+\mathrm{H}\right]^{+}$219.1856, found $\mathrm{m} / \mathrm{z}$ 219.1846

\section{1-(pentan-3-yl)-1-phenylhydrazine (3x)}


Colorless oil; $26 \mathrm{mg}$, Yield: $72 \%$; purified with flash chromatography (hexane/EtOAc $\left.\mathrm{H}_{2}{ }{ }_{N}=20 / 1\right) ;{ }^{1} \mathbf{H}$ NMR $\left(400 \mathrm{MHz} \mathrm{CDCl}_{3}\right) \delta 7.27-7.19(\mathrm{~m}, 2 \mathrm{H}), 6.97(\mathrm{~d}, J=8.6 \mathrm{~Hz}, 2 \mathrm{H})$, $6.70(\mathrm{t}, J=7.2 \mathrm{~Hz}, 1 \mathrm{H}), 3.63-3.49(\mathrm{~m}, 1 \mathrm{H}), 3.20$ (brs, 2H), $1.71-1.59(\mathrm{~m}, 2 \mathrm{H}), 1.58$ - $1.45(\mathrm{~m}, 2 \mathrm{H}), 0.87$ (t, $J=7.4 \mathrm{~Hz}, 6 \mathrm{H}) ;{ }^{13} \mathbf{C} \mathbf{N M R}\left(100 \mathrm{MHz}, \mathrm{CDCl}_{3}\right) \delta 152.8,129.1,116.9,112.2$, 62.8, 24.2, 11.6; HRMS (+ESI) m/z calculated for $\left[\mathrm{C}_{11} \mathrm{H}_{18} \mathrm{~N}_{2}+\mathrm{H}\right]^{+}$179.1543, found 179.1535.

\section{1-(4-fluorophenyl)-1-(1-phenylethyl)hydrazine (3y)}

Colorless oil; $38 \mathrm{mg}$, Yield: 83\%; purified with flash chromatography (hex-<smiles>CC(c1ccccc1)N(N)c1ccc(F)cc1</smilesane/EtOAc $=20 / 1) ;{ }^{1} \mathbf{H}$ NMR $\left(400 \mathrm{MHz}, \mathrm{CDCl}_{3}\right) \delta 7.37-7.23(\mathrm{~m}, 5 \mathrm{H}), 7.04(\mathrm{dd}$, $J=8.4,4.5 \mathrm{~Hz}, 2 \mathrm{H}), 6.95$ (t, $J=8.5 \mathrm{~Hz}, 2 \mathrm{H}), 4.97$ (q, $J=6.6 \mathrm{~Hz}, 1 \mathrm{H}$ ), 3.04 (brs, 2H), $1.52(\mathrm{~d}, J=6.7 \mathrm{~Hz}, 3 \mathrm{H}) ;{ }^{13} \mathbf{C} \mathbf{N M R}\left(100 \mathrm{MHz}, \mathrm{CDCl}_{3}\right) \delta 156.7(\mathrm{~d}, J=236.1$ Hz), 148.0, 140.4, 128.5, 127.6, 127.5, 116.6 (d, $J=7.4$ Hz), 115.4 (d, $J=22.0$

$\mathrm{Hz}), 60.9,14.7$; HRMS (+ESI) m/z calculated for $\left[\mathrm{C}_{14} \mathrm{H}_{15} \mathrm{FN}_{2}+\mathrm{H}\right]^{+}$231.1292, found 231.1293.

\section{1-(4-chlorophenyl)-1-(1-phenylethyl)hydrazine (3z)}

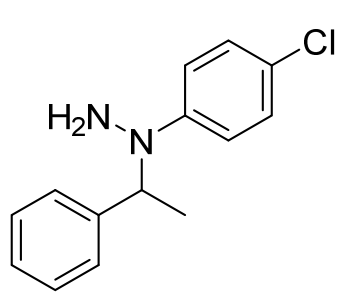

Colorless oil; $41 \mathrm{mg}$, Yield: 82\%; purified with flash chromatography (hexane/EtOAc $=20 / 1) ;{ }^{1} \mathbf{H}$ NMR $\left(300 \mathrm{MHz}, \mathrm{CDCl}_{3}\right): \boldsymbol{\delta} 7.36-7.26(\mathrm{~m}, 5 \mathrm{H}), 7.20(\mathrm{q}$, $J=6.9 \mathrm{~Hz}, 2 \mathrm{H}), 7.09$ (d, $J=7.0 \mathrm{~Hz}, 2 \mathrm{H}), 5.11$ (q, $J=6.9 \mathrm{~Hz}, 1 \mathrm{H}), 3.20$ (brs, 2H), $1.56(\mathrm{t}, J=6.9 \mathrm{~Hz}, 3 \mathrm{H}) ;{ }^{13} \mathbf{C} \mathbf{N M R}\left(150 \mathrm{MHz}, \mathrm{CDCl}_{3}\right): \delta$ 150.1, 140.4, 128.9, 128.6, 127.5, 127.4, 123.1, 115.5, 59.3, 14.7; HRMS (+ESI) m/z calculated for $\left[\mathrm{C}_{14} \mathrm{H}_{15} \mathrm{ClN}_{2}+\mathrm{H}\right]^{+}$247.0997, found 247.0988.

\section{1-(4-nitrophenyl)-1-(1-phenylethyl)hydrazine (3aa)}

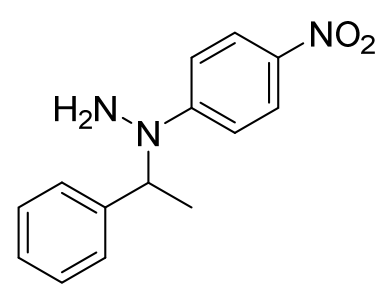

Red oil. $47 \mathrm{mg}$, Yield: $92 \%$; purified with flash chromatography (hexane/EtOAc $=6 / 1) ;{ }^{1} \mathbf{H}$ NMR $\left(600 \mathrm{MHz}, \mathrm{CDCl}_{3}\right): \boldsymbol{\delta} 8.04(\mathrm{~d}, J=9.2 \mathrm{~Hz}, 2 \mathrm{H})$, $7.30(\mathrm{t}, J=7.1 \mathrm{~Hz}, 2 \mathrm{H}), 7.25-7.18(\mathrm{~m}, 3 \mathrm{H}), 7.03(\mathrm{~d}, J=9.2 \mathrm{~Hz}, 2 \mathrm{H}), 5.28$ (q, $J=6.9 \mathrm{~Hz}, 1 \mathrm{H}), 3.34$ (brs, $2 \mathrm{H}), 1.58(\mathrm{~d}, J=6.9 \mathrm{~Hz}, 3 \mathrm{H}) ;{ }^{13} \mathbf{C}$ NMR $(150$ $\left.\mathrm{MHz}, \mathrm{CDCl}_{3}\right): \delta 155.6,139.3,138.0,129.0,128.0,127.0,126.1,111.2,57.6$,

15.3; HRMS (+ESI) m/z calculated for $\left[\mathrm{C}_{14} \mathrm{H}_{15} \mathrm{~N}_{3} \mathrm{O}_{2}+\mathrm{H}\right]^{+} 258.1237$, found 258.1245.

\section{General procedure for organocatalytic direct reductive hydrazination (for aldehyde).}

Under an argon atmosphere, trichlorosilane $(40 \mu \mathrm{L}, 0.40 \mathrm{mmol})$ was added to the mixture of hydrazine $2(0.22 \mathrm{mmol})$ and DMAc $(0.16 \mathrm{~mL}, 1.00 \mathrm{mmol})$ in anhydrous $\mathrm{DCM}$ at $-40{ }^{\circ} \mathrm{C}$, stirring $30 \mathrm{~min}$, and then aldehyde $4(0.20 \mathrm{mmol})$ in DCM was added to the mixture and stirred at the same tempera- 
ture for $24 \mathrm{~h}$. The reaction was quenched with saturated $\mathrm{NH}_{4} \mathrm{Cl}(1 \mathrm{~mL})$, and then the $\mathrm{pH}$ was adjusted to $7 \sim 8$ with saturated $\mathrm{NaHCO}_{3}$, extracted with EtOAc $(3 \times 15 \mathrm{~mL})$. The combined organic layer was washed with brine, dried over anhydrous $\mathrm{MgSO}_{4}$ and concentrated under reduced pressure. Purified with column chromatography (silica gel, hexane/EtOAc) affording pure hydrazine 5.

\section{1-benzyl-1-phenylhydrazine ${ }^{1}$ (5a)}

Colorless oil; $35 \mathrm{mg}$, Yield: $87 \%$; purified with flash chromatography (hex-
$7.23(\mathrm{~m}, 2 \mathrm{H}), 7.09(\mathrm{dd}, J=8.8,0.9 \mathrm{~Hz}, 2 \mathrm{H}), 6.81(\mathrm{t}, J=7.3 \mathrm{~Hz}, 1 \mathrm{H}), 4.59(\mathrm{~s}, 2 \mathrm{H})$, 3.56 (brs, 2H); ${ }^{13} \mathrm{C}$ NMR (100 MHz, $\left.\mathrm{CDCl}_{3}\right) \delta 151.8,137.6,129.1,128.7,127.9,127.4,118.6,113.6$, 60.4 .

\section{1-(3-fluorobenzyl)-1-phenylhydrazine ${ }^{2}(5 b)$}

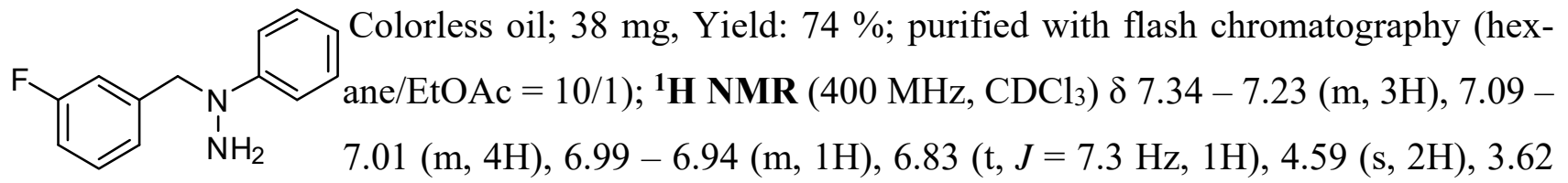
(brs, 2H); ${ }^{13} \mathrm{C}$ NMR $\left(100 \mathrm{MHz}, \mathrm{CDCl}_{3}\right) \delta 163.2(\mathrm{~d}, J=244.7 \mathrm{~Hz}), 151.6,140.7(\mathrm{~d}, J=6.7 \mathrm{~Hz})$, $130.23(\mathrm{~d}, J=8.1 \mathrm{~Hz}), 129.2,123.3(\mathrm{~d}, J=2.8 \mathrm{~Hz}), 118.8,114.6(\mathrm{~d}, J=21.4 \mathrm{~Hz}), 114.3(\mathrm{~d}, J=21.1$ $\mathrm{Hz}), 113.4,60.0(\mathrm{~d}, J=1.7 \mathrm{~Hz})$; HRMS $(+\mathrm{ESI}) \mathrm{m} / \mathrm{z}$ calculated for $\left[\mathrm{C}_{13} \mathrm{H}_{13} \mathrm{FN}_{2}+\mathrm{H}\right]^{+} 217.1136$, found 217.1137.

\section{1-(3-chlorobenzyl)-1-phenylhydrazine (5c)}

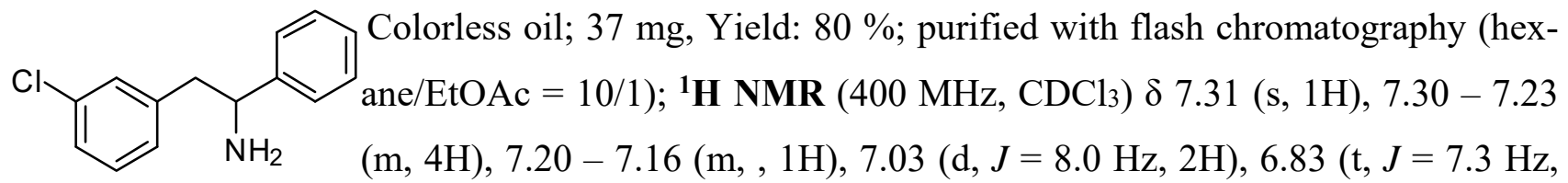
1H), 4.56 (s, 2H), 3.61 (brs, 2H); ${ }^{13} \mathbf{C}$ NMR (100 MHz, $\left.\mathrm{CDCl}_{3}\right) \delta 151.5,140.1,134.6,130.0,129.2$, $127.9,127.6,125.8,118.9,113.4,60.0 ;$ HRMS $(+\mathrm{ESI}) \mathrm{m} / \mathrm{z}$ calculated for $\left[\mathrm{C}_{13} \mathrm{H}_{13} \mathrm{ClN}_{2}+\mathrm{H}\right]^{+}$ 233.0840, found 233.0833 .

\section{1-(3-bromobenzyl)-1-phenylhydrazine (5d)}

Colorless oil; $40 \mathrm{mg}$, Yield: $72 \%$; purified with flash chromatography (hex-
$7.3,1.7 \mathrm{~Hz}, 1 \mathrm{H}), 7.31-7.17(\mathrm{~m}, 4 \mathrm{H}), 7.04(\mathrm{dd}, J=8.8,0.9 \mathrm{~Hz}, 2 \mathrm{H}), 6.83(\mathrm{t}, J$ $=7.3 \mathrm{~Hz}, 1 \mathrm{H}), 4.56(\mathrm{~s}, 2 \mathrm{H}), 3.62(\mathrm{brs}, 2 \mathrm{H}) ;{ }^{13} \mathbf{C ~ N M R}\left(100 \mathrm{MHz}, \mathrm{CDCl}_{3}\right) \delta 151.5,140.4,130.8$, 
$130.5,130.2,129.2,126.3,122.8,118.9,113.4,60.0 ;$ HRMS $(+\mathrm{ESI}) \mathrm{m} / \mathrm{z}$ calculated for $\left[\mathrm{C}_{13} \mathrm{H}_{13} \mathrm{BrN}_{2}\right.$ $+\mathrm{H}]^{+} 277.0335$, found 277.0340 .

\section{1-(3-methoxybenzyl)-1-phenylhydrazine (5e)}

Colorless oil; $36 \mathrm{mg}$, Yield: $80 \%$; purified with flash chromatography (hex3.57 (brs, 2H); ${ }^{13} \mathrm{C}$ NMR (100 MHz, $\left.\mathrm{CDCl}_{3}\right) \delta 156.0,151.8,139.3,129.7,129.1,120.2,118.7,113.7$, 113.3, 112.9, 60.5, 55.2; HRMS (+ESI) $\mathrm{m} / \mathrm{z}$ calculated for $\left[\mathrm{C}_{14} \mathrm{H}_{16} \mathrm{~N}_{2} \mathrm{O}+\mathrm{H}\right]^{+} 229.1335$, found 229.1341.

\section{1-phenyl-1-(3-(trifluoromethyl)benzyl)hydrazine (5f)}

Colorless oil; $45 \mathrm{mg}$, Yield: $84 \%$; purified with flash chromatography (hex$(\mathrm{m}, 2 \mathrm{H}), 7.05(\mathrm{~d}, J=8.0 \mathrm{~Hz}, 2 \mathrm{H}), 6.85$ (t, $J=7.3 \mathrm{~Hz}, 1 \mathrm{H}), 4.63$ (s, 2H), 3.63 (brs, 2H); ${ }^{13} \mathbf{C}$ NMR $\left(100 \mathrm{MHz} \mathrm{CDCl}_{3}\right) \delta 151.6,139.2,131.0,131.0(\mathrm{q}, J=32.0 \mathrm{~Hz}), 129.3,129.2,124.6(\mathrm{q}, J=3.8 \mathrm{~Hz})$, 124.3 (q, $J=3.8 \mathrm{~Hz}), 124.1$ (q, $J=270.7 \mathrm{~Hz}), 119.1,113.5,60.2$; HRMS (+ESI) m/z calculated for $\left[\mathrm{C}_{14} \mathrm{H}_{13} \mathrm{~F}_{3} \mathrm{~N}_{2}+\mathrm{H}\right]^{+}$267.1104, found 267.1109.

\section{1-(4-fluorobenzyl)-1-phenylhydrazine (5g)}

Colorless oil; $35 \mathrm{mg}$, Yield: $82 \%$; purified with flash chromatography (hex-
$7.01(\mathrm{~m}, 4 \mathrm{H}), 6.86(\mathrm{t}, J=7.3 \mathrm{~Hz}, 1 \mathrm{H}), 4.58(\mathrm{~s}, 2 \mathrm{H}), 3.58(\mathrm{brs}, 2 \mathrm{H}) ;{ }^{13} \mathbf{C} \mathbf{N M R}$ $\left(100 \mathrm{MHz} \mathrm{CDCl}_{3}\right) \delta 162.2(\mathrm{~d}, J=243.8 \mathrm{~Hz}), 151.7,133.3(\mathrm{~d}, J=3.2 \mathrm{~Hz}), 129.5(\mathrm{~d}, J=7.9 \mathrm{~Hz})$, 129.2, 118.8, $115.5(\mathrm{~d}, J=21.2 \mathrm{~Hz}), 113.7,59.7$; HRMS $(+\mathrm{ESI}) \mathrm{m} / \mathrm{z}$ calculated for $\left[\mathrm{C}_{13} \mathrm{H}_{13} \mathrm{FN}_{2}+\right.$ $\mathrm{H}]^{+}$217.1136, found 217.1141.

\section{1-(4-chlorobenzyl)-1-phenylhydrazine ${ }^{3}(5 h)$}

Colorless oil; $37 \mathrm{mg}$, Yield: $80 \%$; purified with flash chromatography (hex-
(d, $J=7.9 \mathrm{~Hz}, 1 \mathrm{H}), 6.82(\mathrm{t}, J=7.3 \mathrm{~Hz}, 1 \mathrm{H}), 4.54(\mathrm{~s}, 2 \mathrm{H}), 3.56(\mathrm{brs}, 2 \mathrm{H}) ;{ }^{\mathbf{3}} \mathbf{C}$ NMR $\left(100 \mathrm{MHz}, \mathrm{CDCl}_{3}\right) \delta 151.6,136.2,133.1,129.2,129.2,128.8,118.8,113.6,59.8$; HRMS $(+\mathrm{ESI}) \mathrm{m} / \mathrm{z}$ calculated for $\left[\mathrm{C}_{13} \mathrm{H}_{13} \mathrm{ClN}_{2}+\mathrm{H}\right]^{+} 233.0840$, found 233.0846 . 


\section{1-(4-bromobenzyl)-1-phenylhydrazine ${ }^{6}(5 i)$}

Colorless oil; $46 \mathrm{mg}$, Yield: $84 \%$; purified with flash chromatography
$2 \mathrm{H}), 7.25(\mathrm{t}, J=7.8 \mathrm{~Hz}, 2 \mathrm{H}), 7.16(\mathrm{~d}, J=8.1 \mathrm{~Hz}, 2 \mathrm{H}), 7.02(\mathrm{~d}, J=8.4 \mathrm{~Hz}$, 2H), $6.81(\mathrm{t}, J=7.3 \mathrm{~Hz}, 1 \mathrm{H}), 4.51(\mathrm{~s}, 2 \mathrm{H}), 3.55$ (brs, 2H); ${ }^{13} \mathbf{C} \mathbf{N M R}\left(100 \mathrm{MHz}, \mathrm{CDCl}_{3}\right) \delta 151.6$, 136.8, 131.8, 129.6, 129.2, 121.2, 118.9, 113.6, 59.8; HRMS (+ESI) m/z calculated for $\left[\mathrm{C}_{13} \mathrm{H}_{13} \mathrm{BrN}_{2}\right.$ $+\mathrm{H}]^{+} 277.0335$, found 277.0340 .

\section{1-(4-nitrobenzyl)-1-phenylhydrazine $(5 \mathbf{j})$}

Red solid; $35 \mathrm{mg}$, Yield: $73 \%$; purified with flash chromatography
$8.19(\mathrm{~d}, J=8.2 \mathrm{~Hz}, 2 \mathrm{H}), 7.47(\mathrm{~d}, J=8.2 \mathrm{~Hz}, 2 \mathrm{H}), 7.28(\mathrm{t}, J=7.7 \mathrm{~Hz}, 2 \mathrm{H})$, $6.98(\mathrm{~d}, J=8.3 \mathrm{~Hz}, 2 \mathrm{H}), 6.85(\mathrm{t}, J=7.2 \mathrm{~Hz}, 1 \mathrm{H}), 4.71(\mathrm{~s}, 2 \mathrm{H}), 3.75$ (brs, 2H); ${ }^{13} \mathbf{C}$ NMR (100 MHz, $\left.\mathrm{CDCl}_{3}\right) \delta 151.3,147.3,146.1,129.4,128.3,123.9,119.2,113.1,59.8 ;$ HRMS (+ESI) m/z calculated for $\left[\mathrm{C}_{13} \mathrm{H}_{13} \mathrm{~N}_{3} \mathrm{O}_{2}+\mathrm{H}\right]^{+} 244.1081$, found 244.1085 .

\section{1-(4-isopropylbenzyl)-1-phenylhydrazine (5k)}<smiles>CC(C)c1ccc(CN(N)c2ccccc2)cc1</smiles>

Colorless oil; $37 \mathrm{mg}$, Yield: $77 \%$; purified with flash chromatography (hexane/EtOAc = 15/1); ${ }^{1} \mathbf{H}$ NMR $\left(400 \mathrm{MHz}, \mathrm{CDCl}_{3}\right) \delta 7.31-7.16(\mathrm{~m}, 6 \mathrm{H})$, $7.11(\mathrm{~d}, J=8.3 \mathrm{~Hz}, 2 \mathrm{H}), 6.80(\mathrm{t}, J=7.2 \mathrm{~Hz}, 1 \mathrm{H}), 4.55$ (s, 2H), 3.52 (brs, 2H), $2.93-2.86(\mathrm{~m}, 1 \mathrm{H}), 1.24(\mathrm{~d}, J=6.9 \mathrm{~Hz}, 6 \mathrm{H}) ;{ }^{13} \mathbf{C ~ N M R}\left(100 \mathrm{MHz}, \mathrm{CDCl}_{3}\right)$ $\delta 151.9,148.19,134.8,129.0,128.07,126.7,118.5,113.72,60.1,33.8,24.0 ;$ HRMS $(+\mathrm{ESI}) \mathrm{m} / \mathrm{z}$ calculated for $\left[\mathrm{C}_{16} \mathrm{H}_{20} \mathrm{~N}_{2}+\mathrm{H}\right]^{+} 241.1699$, found 241.1705 .

\section{1-(4-methoxybenzyl)-1-phenylhydrazine ${ }^{3}(51)$}

Colorless oil; $32 \mathrm{mg}, \quad$ Yield: $71 \%$; purified with flash chromatography
$7.10(\mathrm{~d}, J=8.2 \mathrm{~Hz}, 2 \mathrm{H}), 6.86(\mathrm{~d}, J=8.5 \mathrm{~Hz}, 2 \mathrm{H}), 6.81(\mathrm{t}, J=7.2 \mathrm{~Hz}, 1 \mathrm{H})$, 4.50 (s, 2H), 3.79 (s, 3H), 3.48 (brs, 2H); $\left.{ }^{13} \mathbf{C ~ N M R ~ ( 1 0 0 ~ M H z , ~} \mathrm{CDCl}_{3}\right) \delta$ 159.0, 151.8, 129.4, 129.3, 129.1, 118.6, 114.1, 113.9, 59.8, 55.3; HRMS (+ESI) m/z calculated for $\left[\mathrm{C}_{14} \mathrm{H}_{16} \mathrm{~N}_{2} \mathrm{O}+\mathrm{H}\right]^{+} 229.1335$, found 229.1341 . 


\section{1-(naphthalen-2-ylmethyl)-1-phenylhydrazine (5m)}

White solid; $41 \mathrm{mg}$, Yield: $82 \%$; purified with flash chromatography
(hexane/EtOAc $=10 / 1)$; m.p. $94-96{ }^{\circ} \mathrm{C} ;{ }^{1} \mathbf{H}$ NMR $\left(400 \mathrm{MHz}, \mathrm{CDCl}_{3}\right) \delta$ $7.26(\mathrm{t}, J=7.8 \mathrm{~Hz}, 2 \mathrm{H}), 7.12(\mathrm{~d}, J=8.5 \mathrm{~Hz}, 2 \mathrm{H}), 6.82$ (t, $J=7.2 \mathrm{~Hz}, 1 \mathrm{H}), 4.70$ (s, 2H), 3.56 (brs, $2 \mathrm{H}) ;{ }^{13} \mathrm{C}$ NMR $\left(100 \mathrm{MHz}, \mathrm{CDCl}_{3}\right) \delta 151.9,135.2,133.5,132.9,129.2,128.6,127.8,127.8,126.7$, 126.3, 126.0, 125.9, 118.7, 113.8, 60.8. HRMS (+ESI) $\mathrm{m} / \mathrm{z}$ calculated for $\left[\mathrm{C}_{14} \mathrm{H}_{16} \mathrm{~N}_{2} \mathrm{O}+\mathrm{H}\right]^{+}$ 249.1386, found 249.1392.

\section{1-(naphthalen-1-ylmethyl)-1-phenylhydrazine (5n)}

Colorless oil; $32 \mathrm{mg}$, Yield: $65 \%$; purified with flash chromatography (hex-
$7.87(\mathrm{~m}, 1 \mathrm{H}), 7.81(\mathrm{~d}, \mathrm{~J}=7.9 \mathrm{~Hz}, 1 \mathrm{H}), 7.54-7.48(\mathrm{~m}, 2 \mathrm{H}), 7.44-7.38(\mathrm{~m}$, 2H), $7.29(\mathrm{dd}, J=8.4,7.5 \mathrm{~Hz}, 2 \mathrm{H}), 7.16(\mathrm{~d}, J=8.0 \mathrm{~Hz}, 2 \mathrm{H}), 6.84(\mathrm{t}, J=7.2 \mathrm{~Hz}, 1 \mathrm{H}), 4.98(\mathrm{~s}, 2 \mathrm{H})$, 3.55 (brs, 2H); ${ }^{13} \mathbf{C}$ NMR (100 MHz, $\left.\mathrm{CDCl}_{3}\right) \delta 151.8,134.0,132.6,131.8,129.2,128.8,128.3,126.4$, 126.1, 126.0, 125.5, 123.4, 118.6, 113.5, 58.5; HRMS (+ESI) m/z calculated for $\left[\mathrm{C}_{17} \mathrm{H}_{16} \mathrm{~N}_{2}+\mathrm{H}\right]^{+}$ 249.1386, found 249.1382 .

\section{1-cinnamyl-1-phenylhydrazine $\mathrm{e}^{3,4}(50)$}

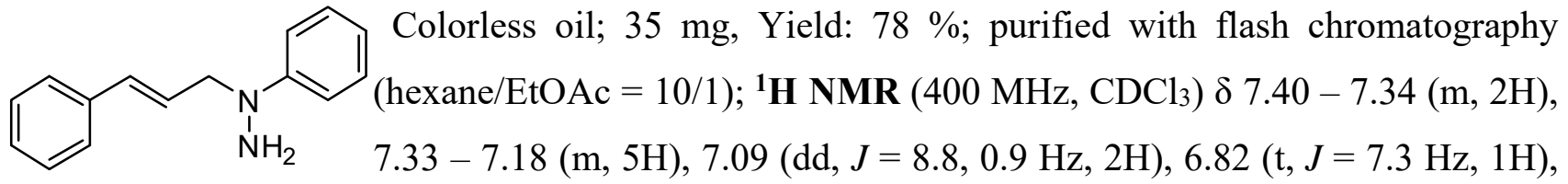
$6.63(\mathrm{~d}, J=15.9 \mathrm{~Hz}, 1 \mathrm{H}), 6.27$ (dt, $J=15.9,6.3 \mathrm{~Hz}, 1 \mathrm{H}), 4.19$ (dd, $J=6.3,1.3 \mathrm{~Hz}, 2 \mathrm{H}), 3.65$ (brs, 2H); ${ }^{13}$ C NMR (100 MHz, $\left.\mathrm{CDCl}_{3}\right) \delta 151.42,136.6,133.9,129.1,128.6,127.7,126.4,124.1,118.7$, 113.9, 58.5; HRMS (+ESI) m/z calculated for $\left[\mathrm{C}_{15} \mathrm{H}_{16} \mathrm{~N}_{2}+\mathrm{H}\right]^{+} 225.1386$, found 225.1392 .

\section{1-phenyl-1-(3-phenylpropyl)hydrazine ${ }^{3}(5 p)$}

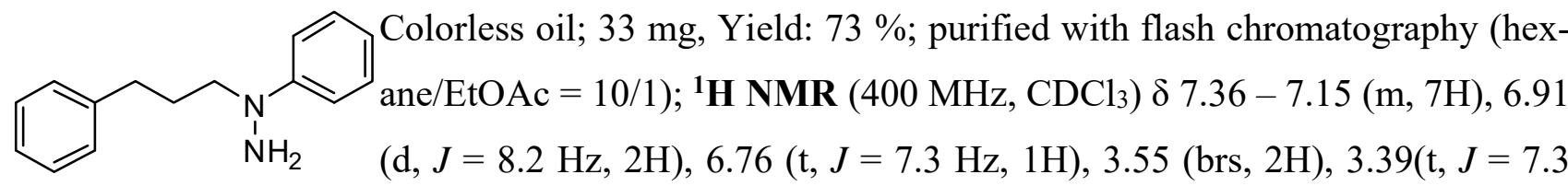
$\mathrm{Hz}, 2 \mathrm{H}), 2.69$ (t, $J=7.7 \mathrm{~Hz}, 2 \mathrm{H}), 2.06-1.89(\mathrm{~m}, 2 \mathrm{H}) ;{ }^{13} \mathbf{C} \mathbf{N M R}\left(100 \mathrm{MHz}, \mathrm{CDCl}_{3}\right) \delta 151.8,141.8$, 129.1, 128.4, 128.4, 125.9, 118.2, 113.2, 55.1, 33.2, 27.6; HRMS (+ESI) m/z calculated for $\left[\mathrm{C}_{15} \mathrm{H}_{18} \mathrm{~N}_{2}+\mathrm{H}\right]^{+} 227.1543$, found 227.1548. 


\section{1-(cyclohexylmethyl)-1-phenylhydrazine (5q)}

$\overbrace{\substack{1 \\ \mathrm{NH}_{2}}}^{N}$ Colorless oil; $33 \mathrm{mg}$, Yield: $81 \%$; purified with flash chromatography (hexane/EtOAc $=15 / 1) ;{ }^{1} \mathbf{H}$ NMR $\left(400 \mathrm{MHz}, \mathrm{CDCl}_{3}\right) \delta 7.23(\mathrm{t}, J=6.7 \mathrm{~Hz}, 2 \mathrm{H}), 6.93$ $(\mathrm{d}, J=8.4 \mathrm{~Hz}, 2 \mathrm{H}), 6.74$ (t, $J=7.2 \mathrm{~Hz}, 1 \mathrm{H}), 3.57$ (brs, 2H), 3.19 (d, $J=6.8 \mathrm{~Hz}$, 2H), $1.93-1.61(\mathrm{~m}, 6 \mathrm{H}), 1.35-1.13(\mathrm{~m}, 3 \mathrm{H}), 1.07-0.92(\mathrm{~m}, 2 \mathrm{H}) ;{ }^{13} \mathbf{C} \mathbf{N M R}\left(100 \mathrm{MHz}, \mathrm{CDCl}_{3}\right) \delta$ $152.2,129.0,117.5,112.5,63.2,36.4,31.3,26.6,26.0 ;$ HRMS $(+\mathrm{ESI}) \mathrm{m} / \mathrm{z}$ calculated for $\left[\mathrm{C}_{13} \mathrm{H}_{20} \mathrm{~N}_{2}\right.$ $+\mathrm{H}]^{+}$205.1699, found 205.1705.

\section{1-octyl-1-phenylhydrazine ${ }^{5}(5 r)$}

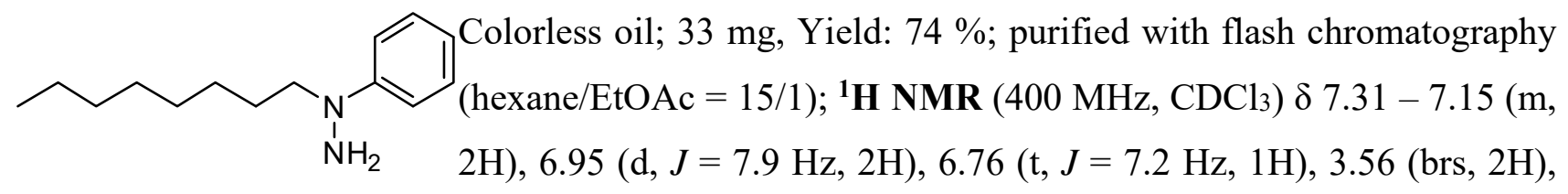
$3.36(\mathrm{t}, J=7.5 \mathrm{~Hz}, 2 \mathrm{H}), 1.72-1.53(\mathrm{~m}, 2 \mathrm{H}), 1.44-1.18(\mathrm{~m}, 10 \mathrm{H}), 0.88(\mathrm{t}, J=6.8 \mathrm{~Hz}, 3 \mathrm{H}) ;{ }^{13} \mathbf{C}$ NMR $\left(100 \mathrm{MHz}, \mathrm{CDCl}_{3}\right) \delta 151.8,129.0,117.9,113.1,55.8,31.9,29.6,29.3,27.1,25.8,22.7,14.1$; HRMS (+ESI) m/z calculated for $\left[\mathrm{C}_{14} \mathrm{H}_{24} \mathrm{~N}_{2}+\mathrm{H}\right]^{+} 221.2012$, found 221.2018 .

\section{General procedure for asymmetric catalytic direct reductive hydrazination}

Under an argon atmosphere, the ketone $1(0.20 \mathrm{mmol})$ and hydrazine $2(0.20 \mathrm{mmol})$ and $6^{7}(6.9 \mathrm{mg}$, $0.02 \mathrm{mmol}$ ) was added in anhydrous DCM and stirred at $-40{ }^{\circ} \mathrm{C}$ for $10 \mathrm{~min}$, and then trichlorosilane (40 $\mu \mathrm{L}, 0.40 \mathrm{mmol}$ ) was added. The reaction was stirred at this temperature for $36 \mathrm{~h}$, and then quenched with saturated $\mathrm{NH}_{4} \mathrm{Cl}(1 \mathrm{~mL})$. The $\mathrm{pH}$ of the mixture was adjusted to $7 \sim 8$ with saturated $\mathrm{NaHCO}_{3}$, and then extracted with EtOAc $(3 \times 15 \mathrm{~mL})$. The combined organic layer were washed with brine, dried over anhydrous $\mathrm{MgSO}_{4}$ and concentrated under reduced pressure. Purified with column chromatography (silica gel, hexane/EtOAc) affording pure hydrazine 3a.

6: Prepared with reported method. ${ }^{7}$ White solids, yield 90\%, ${ }^{1} \mathbf{H}$ NMR $\left(400 \mathrm{MHz}, \mathrm{CDCl}_{3}\right) \delta 7.32(\mathrm{~m}$, 2H), 7.28 (s, 1H), 7.27 (d, $J=2.4 \mathrm{~Hz}, 1 \mathrm{H}), 4.34$ (dd, $J=4.8 \mathrm{~Hz}, J=13.8 \mathrm{~Hz}, 2 \mathrm{H}), 4.25$ (dd, $J=7.6$ $\mathrm{Hz}, J=13.8 \mathrm{~Hz}, 2 \mathrm{H}), 3.47-2.49(\mathrm{~m}, 2 \mathrm{H}), 1.25(\mathrm{~s}, 18 \mathrm{H})$.

3a: $[\mathbf{a}] \mathbf{b}^{\mathbf{2 0}}=-164\left(\mathrm{c}=0.5, \mathrm{CDCl}_{3}\right) ;{ }^{\mathbf{1}} \mathbf{H} \mathbf{N M R}\left(300 \mathrm{MHz}, \mathrm{CDCl}_{3}\right): \boldsymbol{\delta} 7.35-7.29(\mathrm{~m}, 7 \mathrm{H}), 7.10(\mathrm{~d}, J=$ $7.9 \mathrm{~Hz}, 2 \mathrm{H}), 6.82$ (t, $J=7.2 \mathrm{~Hz}, 1 \mathrm{H}), 5.15$ (q, $J=6.8 \mathrm{~Hz}, 1 \mathrm{H}), 3.12$ (brs, 2H), 1.57 (d, $J=6.8 \mathrm{~Hz}$, $3 \mathrm{H}) ;{ }^{13} \mathrm{C}$ NMR $\left(75 \mathrm{MHz}, \mathrm{CDCl}_{3}\right): \delta$ 151.3, 140.9, 129.0, 128.4, 127.4, 127.2, 118.3, 114.0, 58.9, 14.6. The enantiomers were analyzed by HPLC using a chiral OD-H column (n-heptane/2-propanol 
$=90 / 10$, flow rate $=1.0 \mathrm{~mL} / \mathrm{min}$, wavelength $=254 \mathrm{~nm}$ ); minor enantiomer: $\mathrm{t}_{\mathrm{R}}=10.1$ min, major enantiomer: $\mathrm{t}_{\mathrm{R}}=14.7 \mathrm{~min}$.

\section{References}

(1) Muller, S.; Webber, M. J.; List, B. J. Am. Chem. Soc. 2011, 133, 18534.

(2) Yeo, S. J.; Liu, Y.; Wang, X. Tetrahedron 2012, 68, 813.

(3) Nara, S.; Sakamoto, T.; Miyazawa, E.; Kikugawa, Y. Syn. Comm. 2003, 33, 87.

(4) Tiecco, M.; Testaferri, L.; Marini, F.; Bagnoli, L.; Santi, C.; Temperini, A. Tetrahedron 1997, 53, 4441.

(5) Lerch, U.; König, J. Synthesis 1983, 1983, 157.

(6) Schammel, A. W.; Boal, B. W.; Zu, L.; Mesganaw, T.; Garg, N. K., Tetrahedron 2010, 66, 4687-4695.

(7) Pei, D.; Zhang, Y.; Wei, S. Y.; Wang, M.; Sun, J., Adv. Synth. Catal. 2008, 350, 619-623. 
${ }^{1} \mathrm{H}$ NMR of $\mathbf{3 a}$

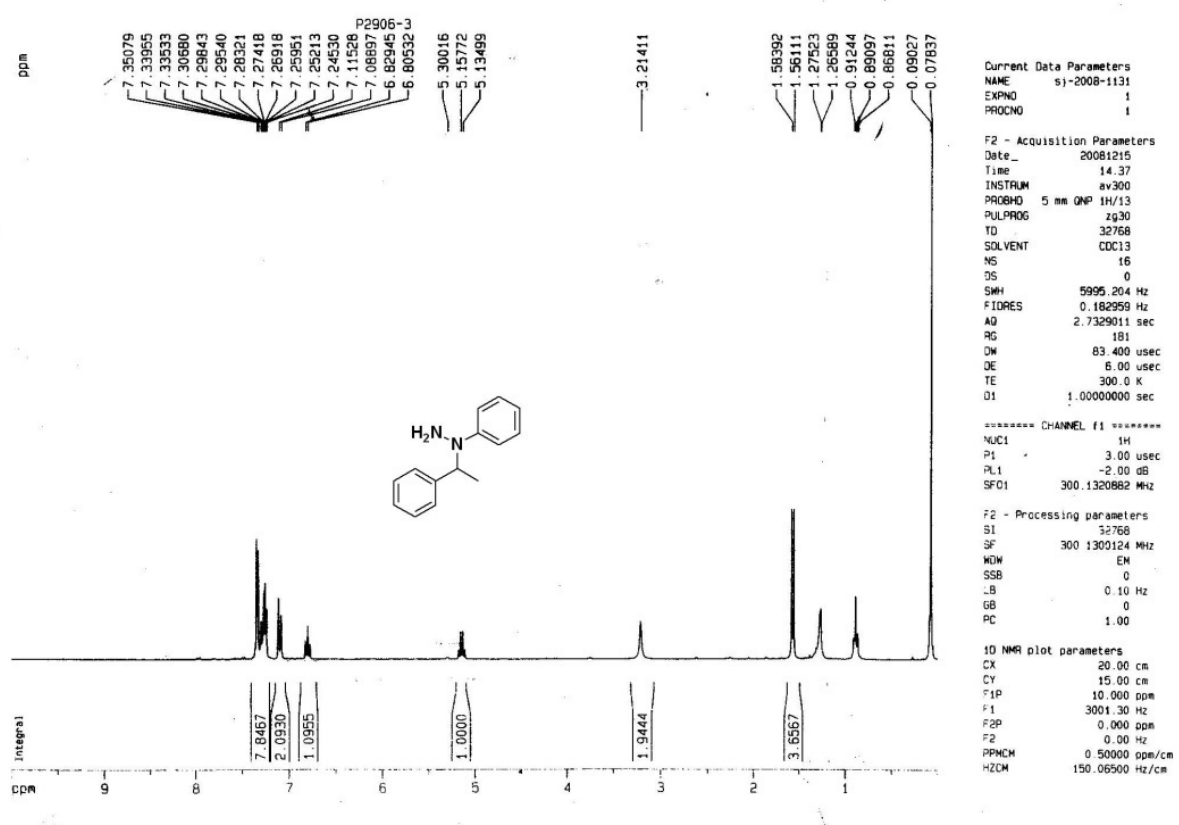

${ }^{13}$ C NMR of $3 a$

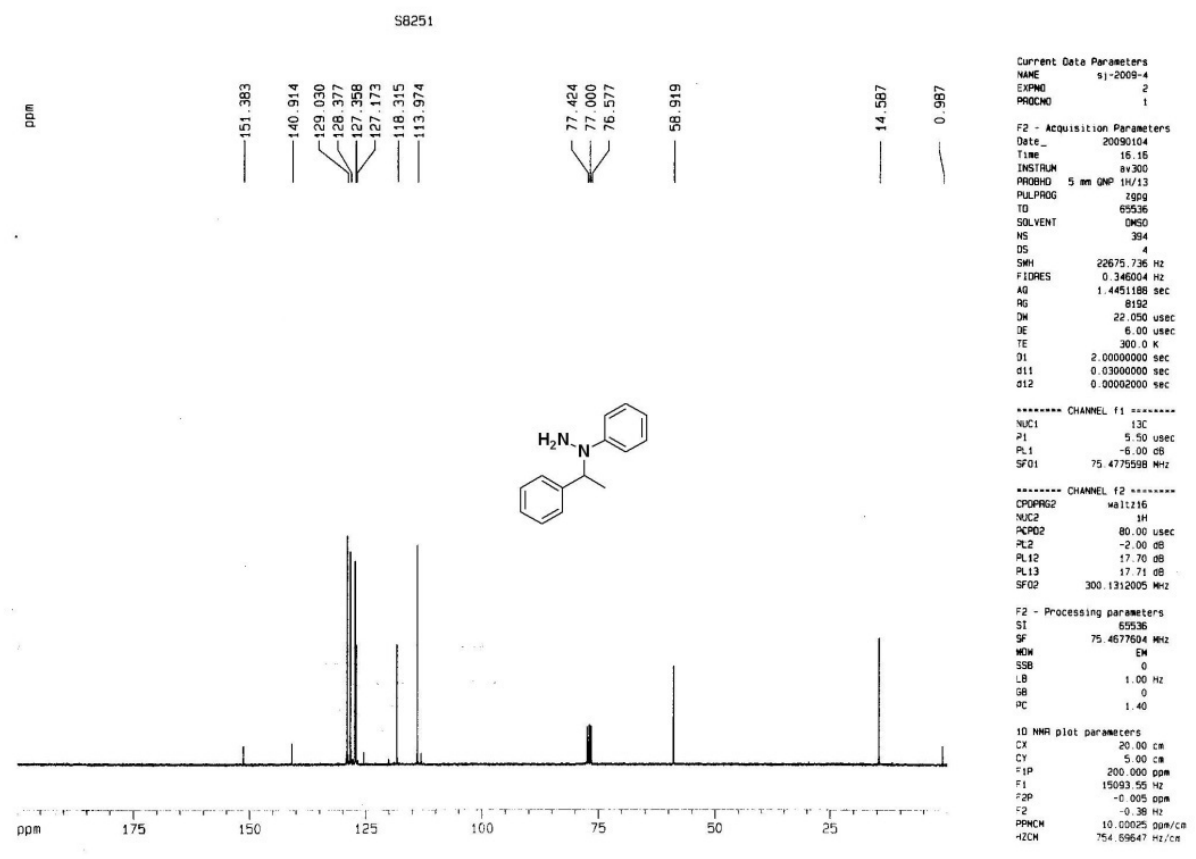


${ }^{1} \mathrm{H}$ NMR of $\mathbf{3 b}$

Bruker Avance 600 probe: 13C-1H DUL TE: 300Ksample: s21391 solvent CDCL3 spectrum: IH

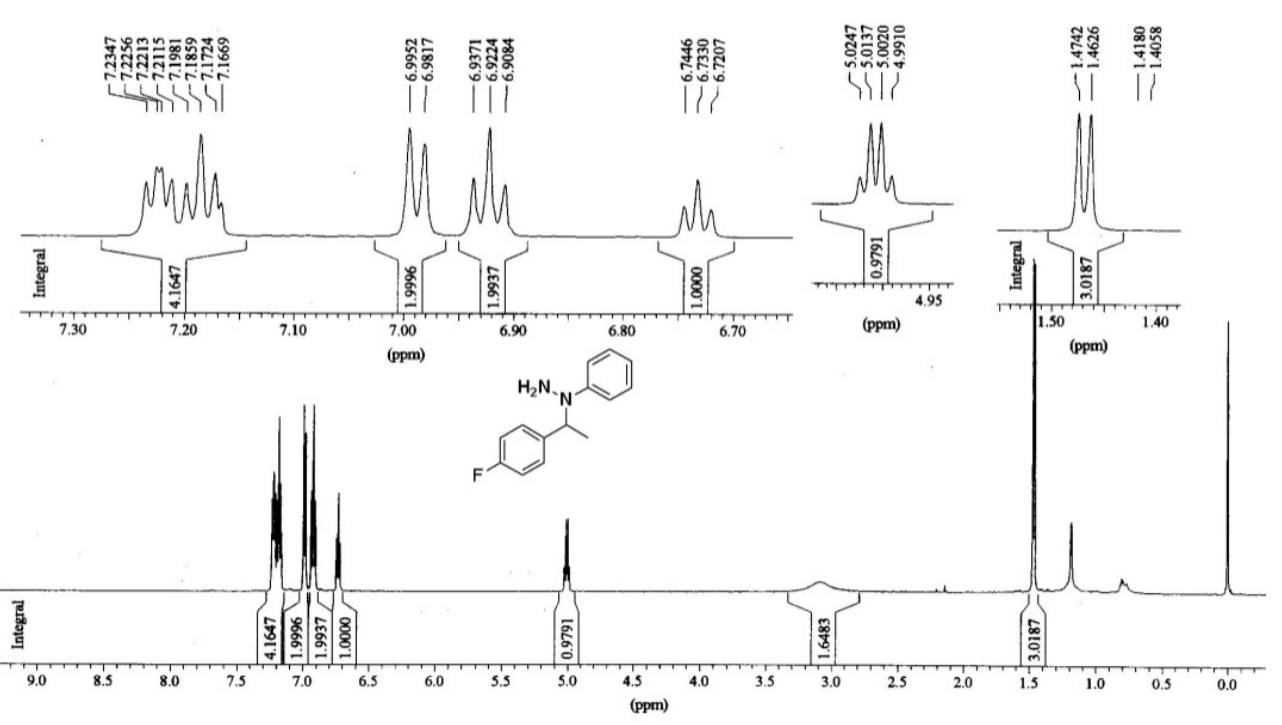

${ }^{13}$ C NMR of $3 b$

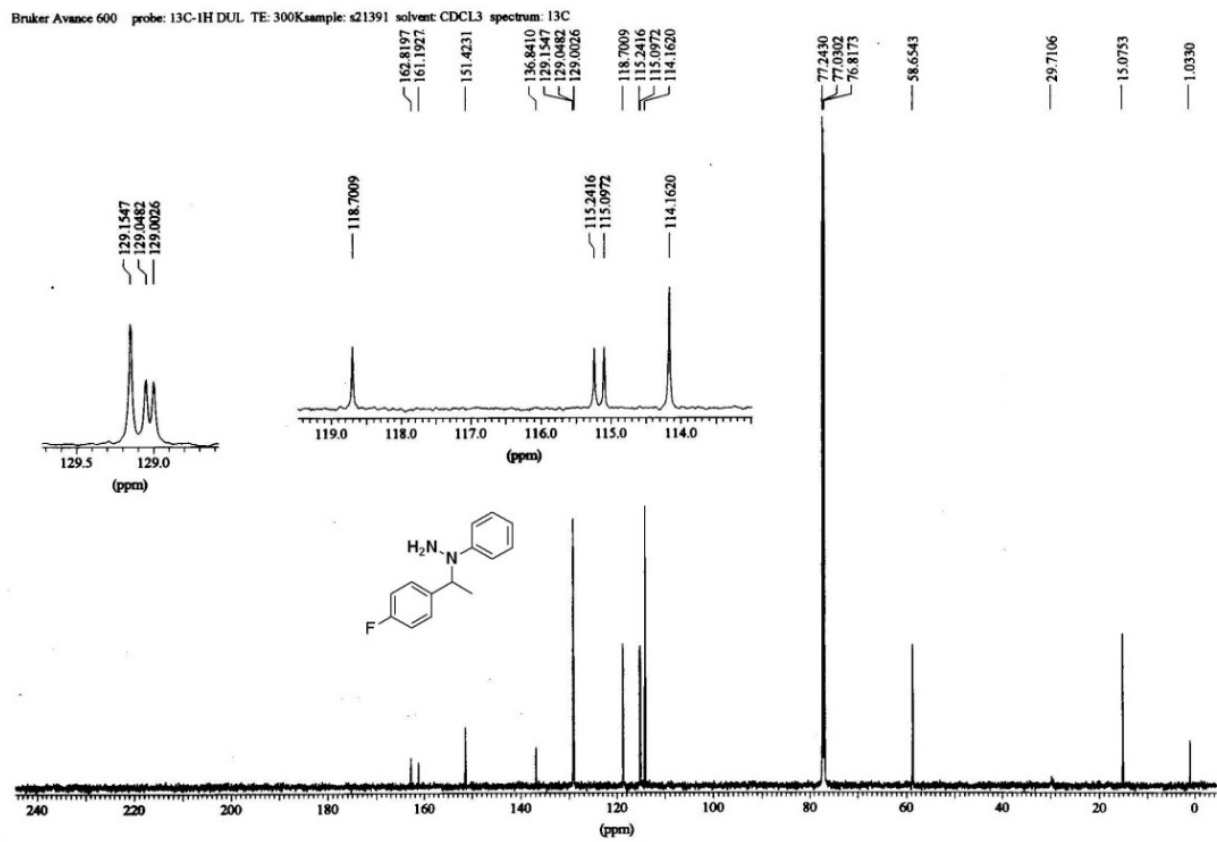


${ }^{1} \mathrm{H}$ NMR of $\mathbf{3 c}$

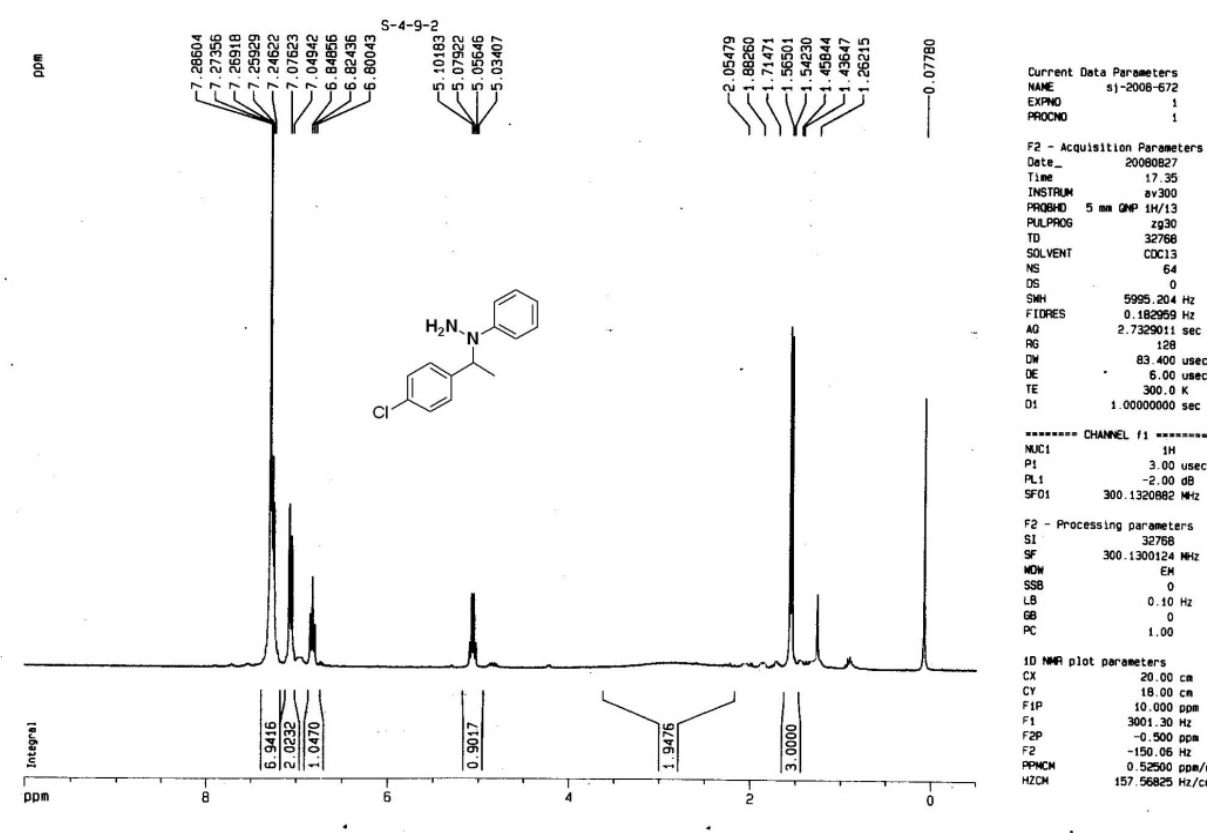

${ }^{13}$ C NMR of of $3 c$

S8182
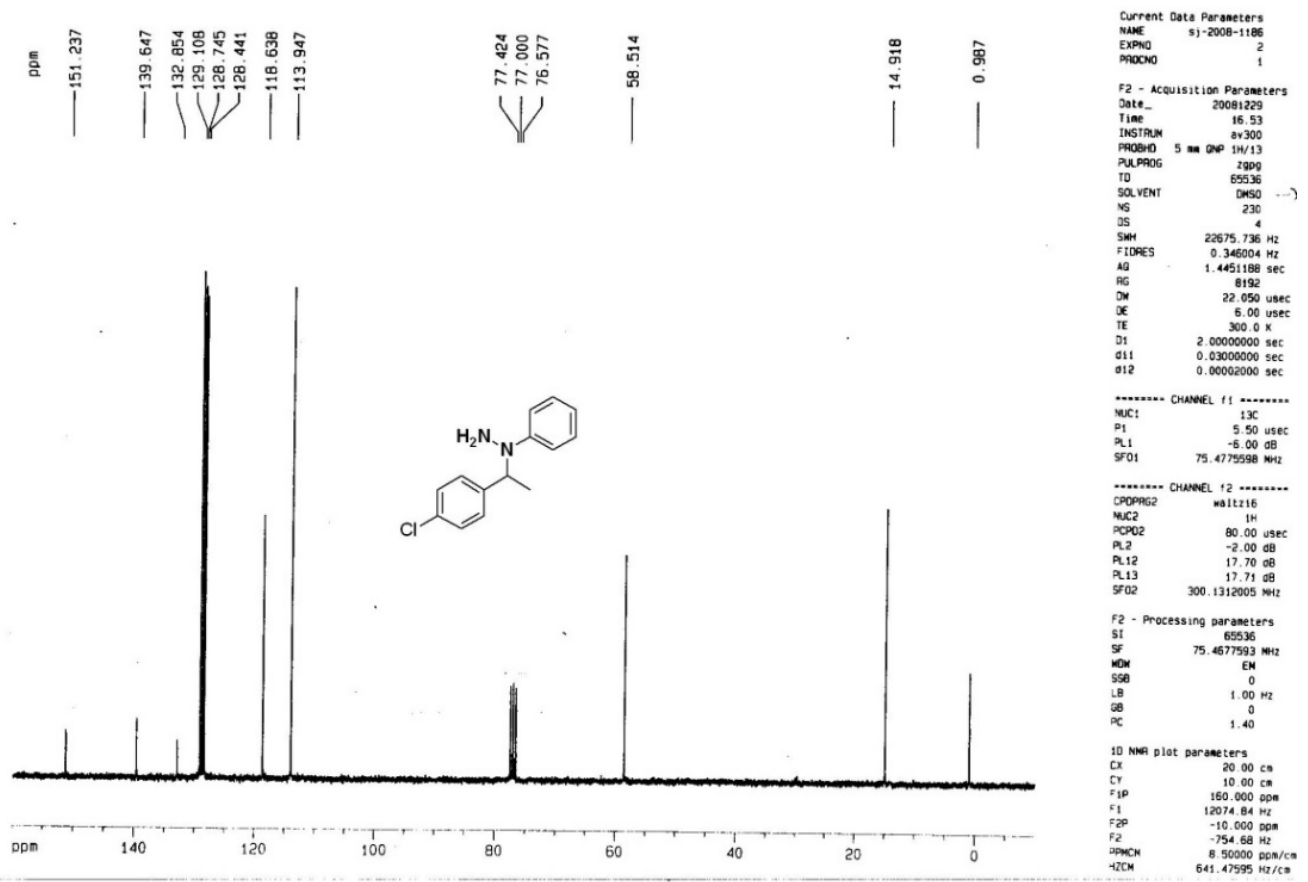
${ }^{1} \mathrm{H}$ NMR of 3d

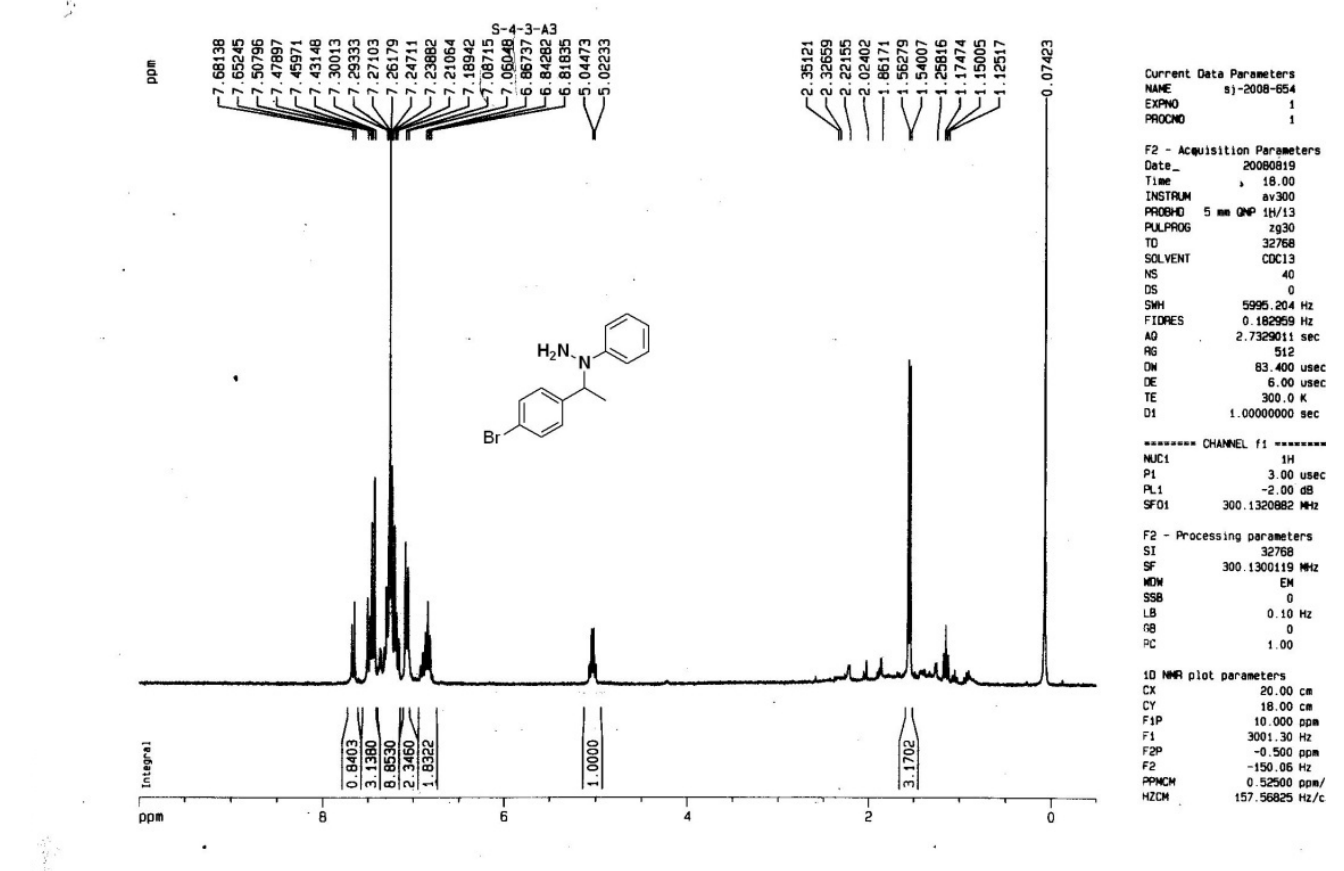

${ }^{13}$ C NMR of 3d
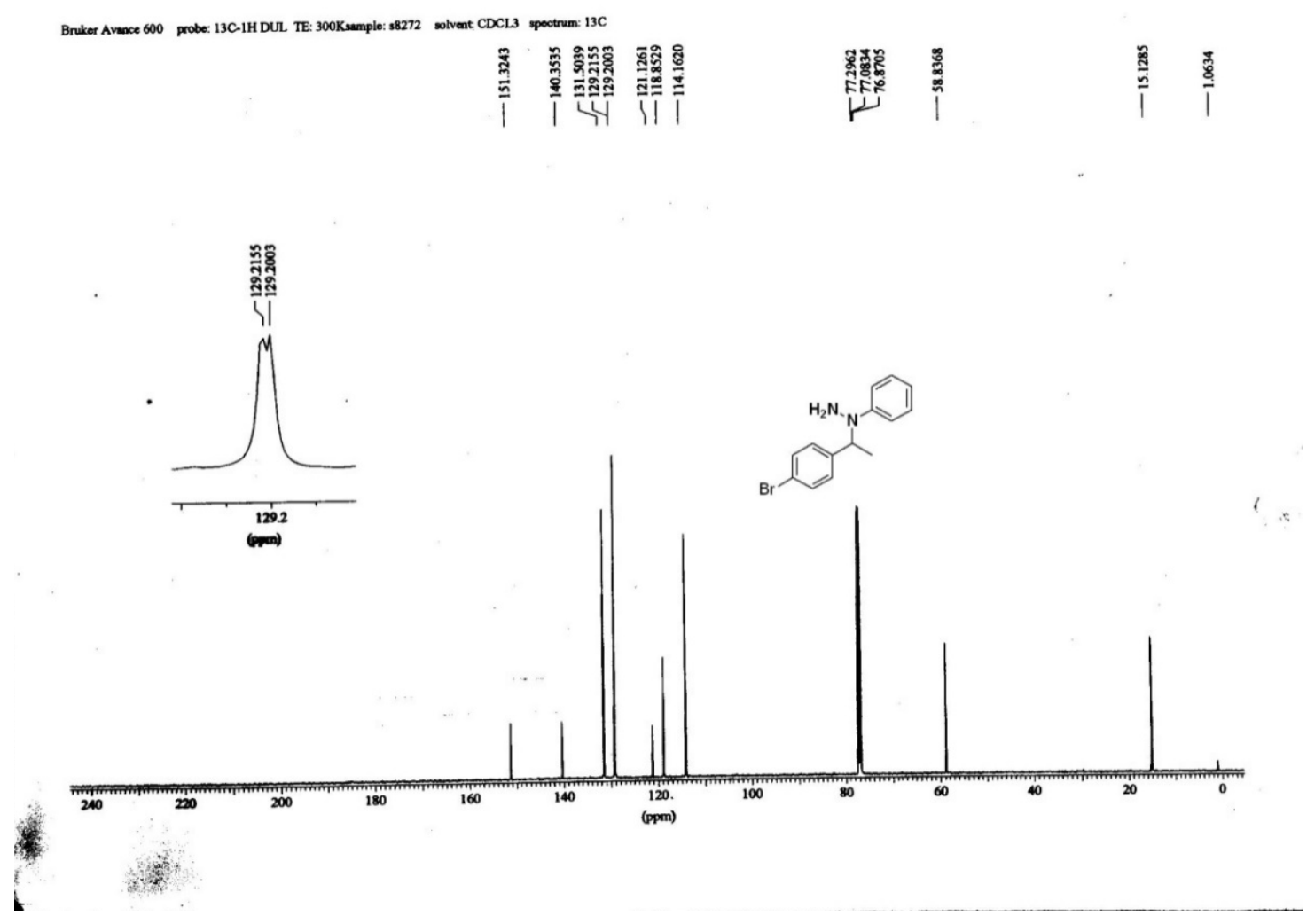
${ }^{1} \mathrm{H}$ NMR of $\mathbf{3 e}$

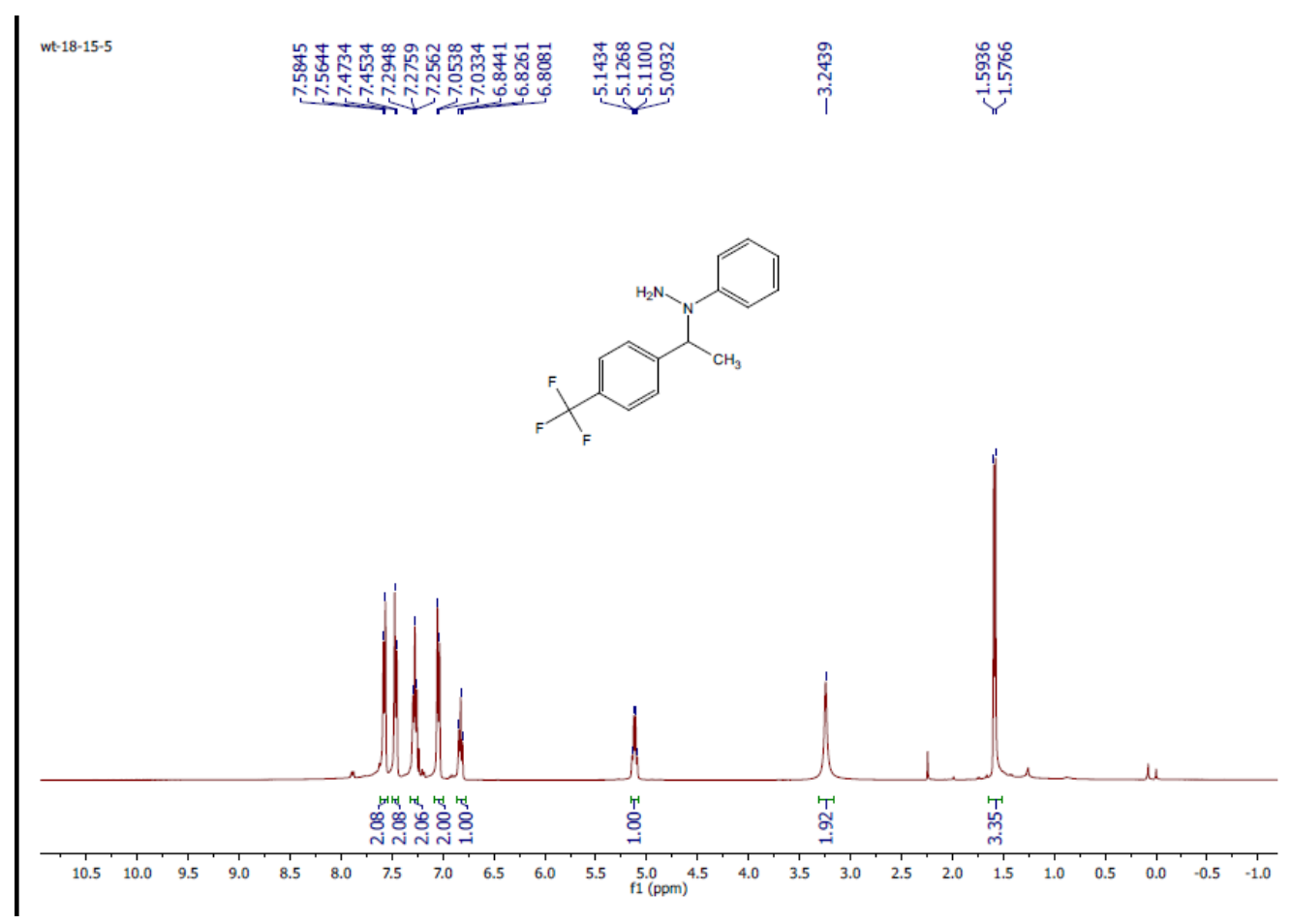

${ }^{1} \mathrm{H}$ NMR of $\mathbf{3 e}$

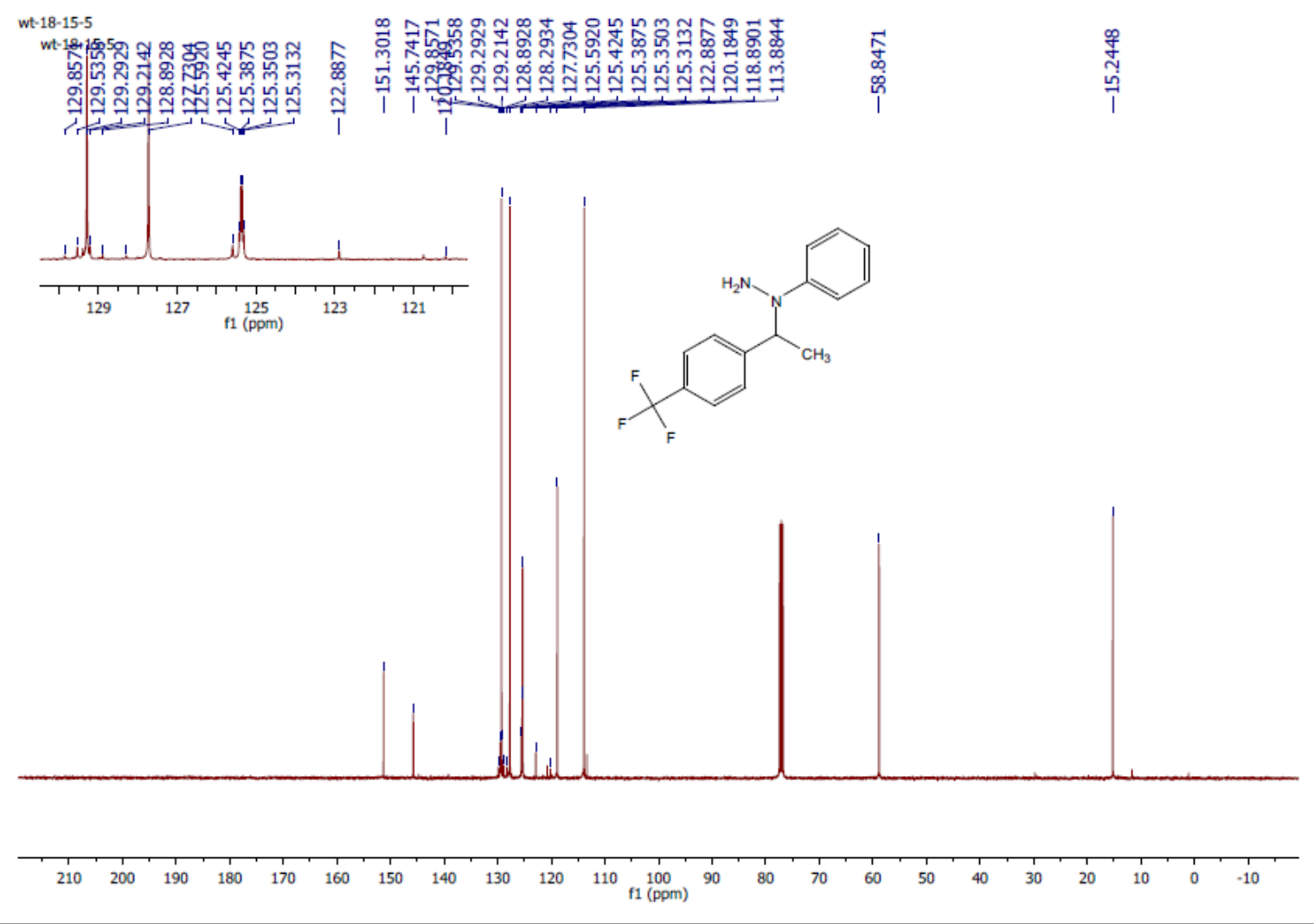


${ }^{1} \mathrm{H}$ NMR of $\mathbf{3 f}$

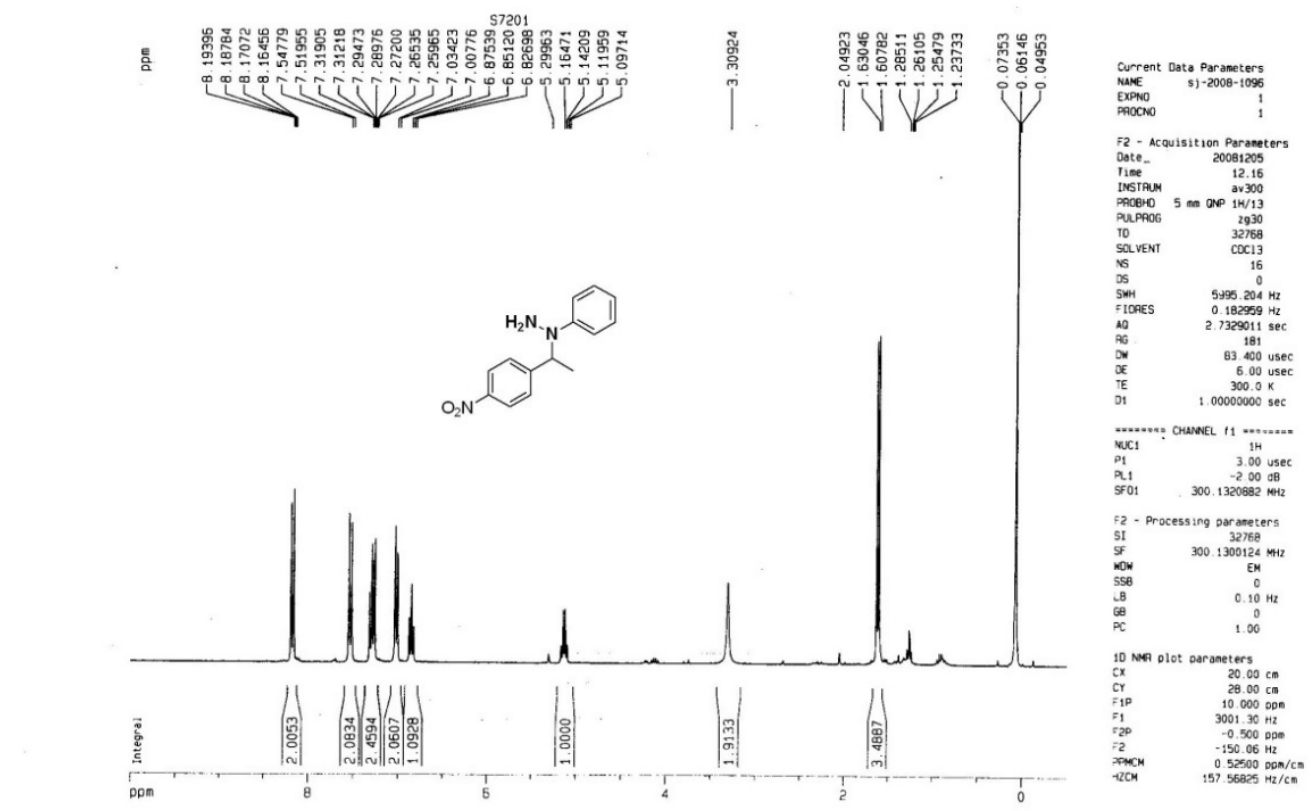

1

${ }^{13}$ C NMR of $3 f$

Bruker Avance 600 probe: $13 \mathrm{C}-1 \mathrm{H}$ DUL, TE: $300 \mathrm{~K}$ sample: 88331 solvent: CDCL3 spectrum: $13 \mathrm{C}$

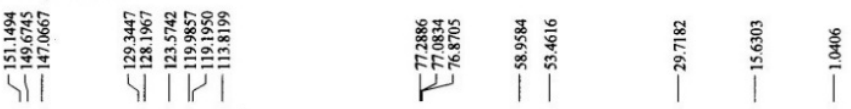
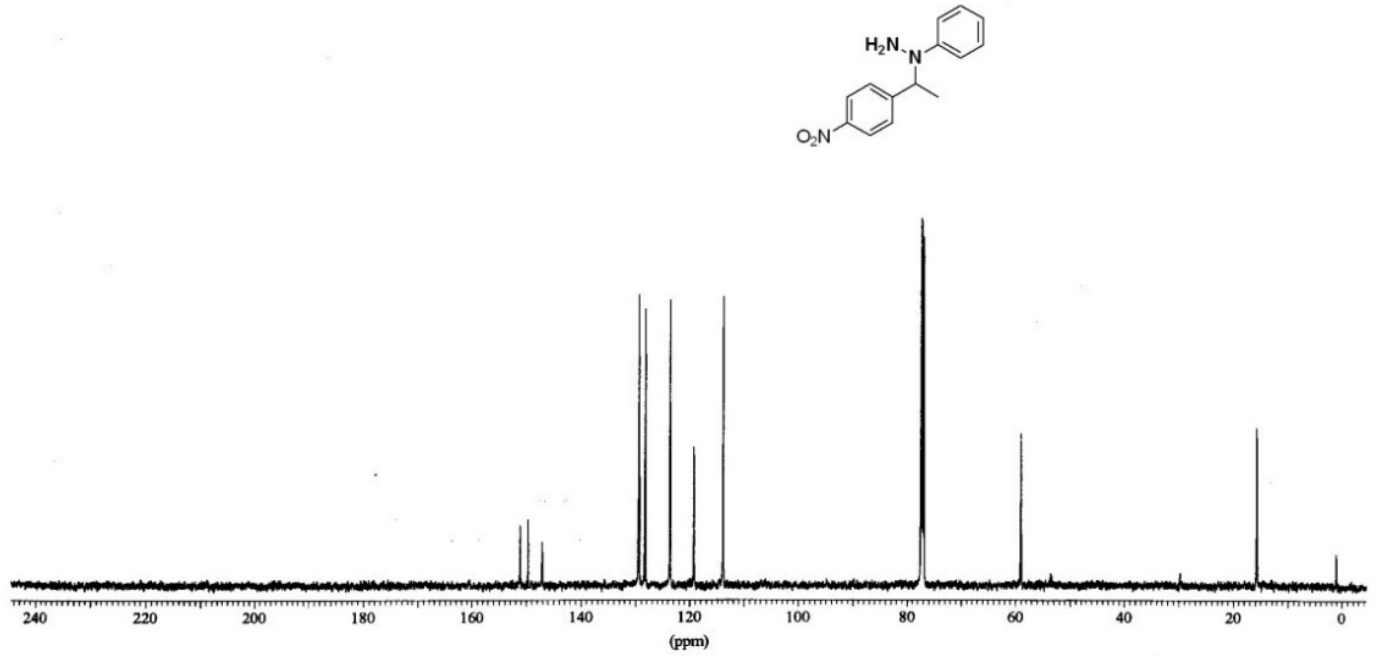
${ }^{1} \mathrm{H}$ NMR of $\mathbf{3 g}$

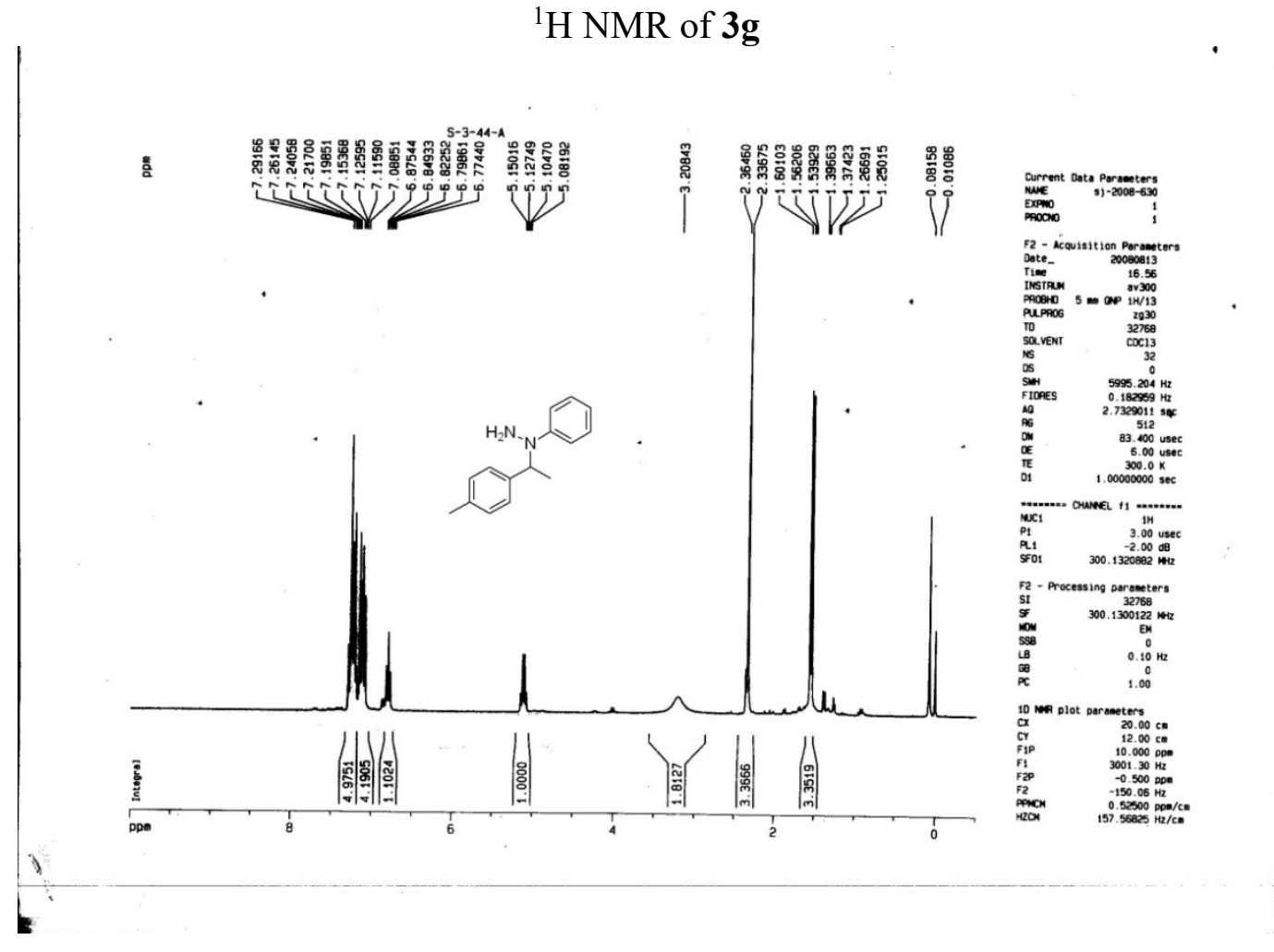

\section{${ }^{13}$ C NMR of $3 g$}
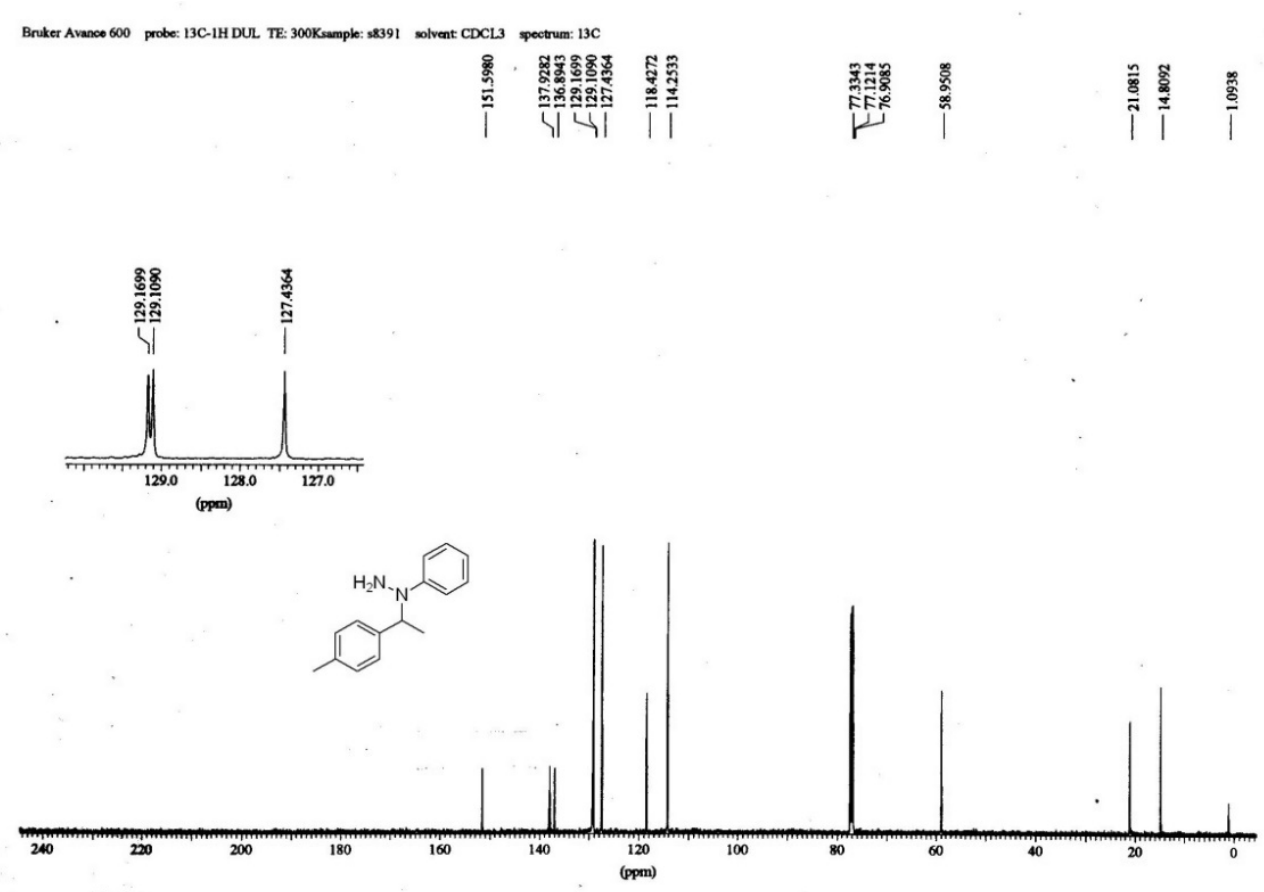
${ }^{1} \mathrm{H}$ NMR of $\mathbf{3 h}$

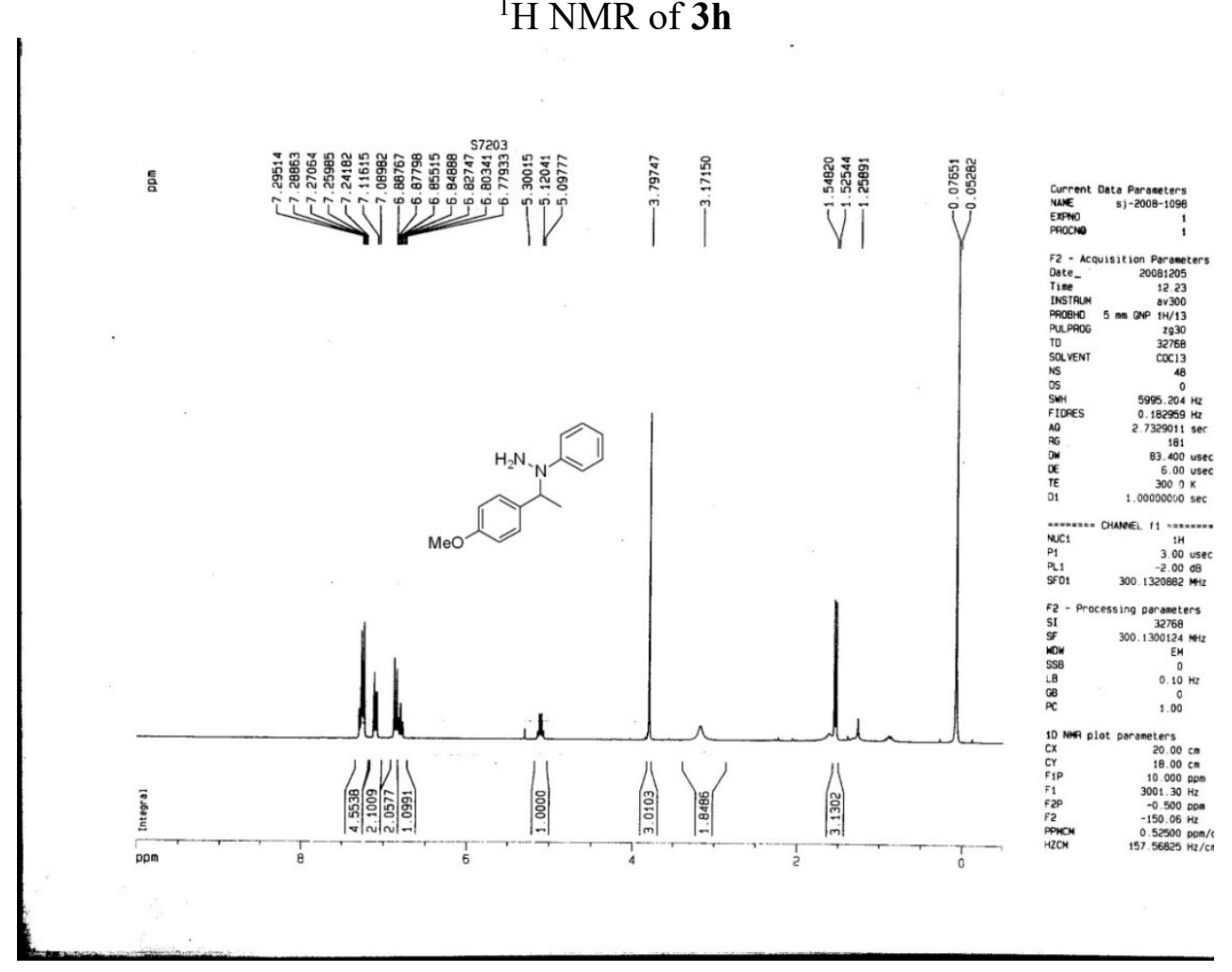

${ }^{13}$ C NMR of $3 h$

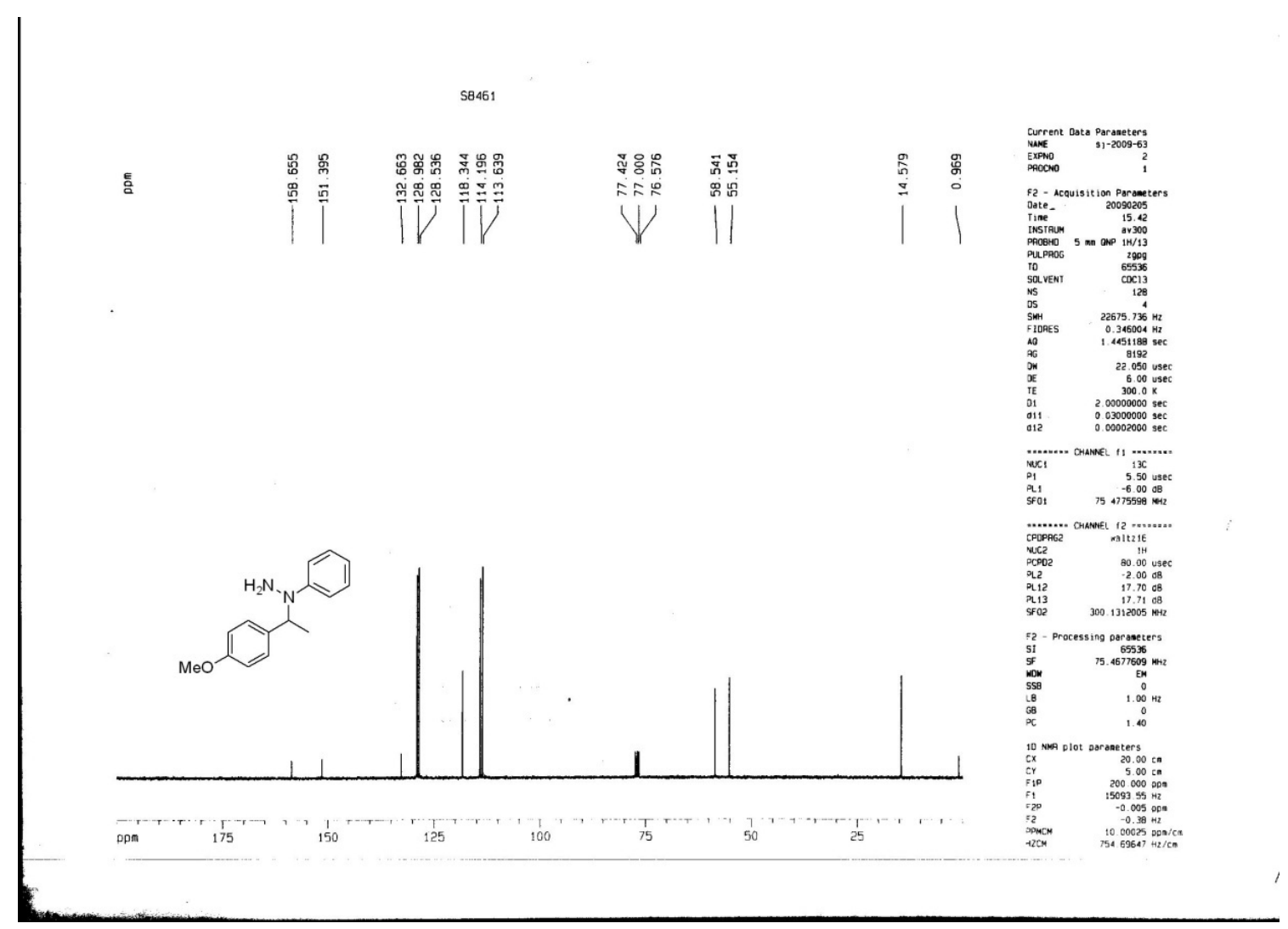


${ }^{1} \mathrm{H}$ NMR of $\mathbf{3 i}$

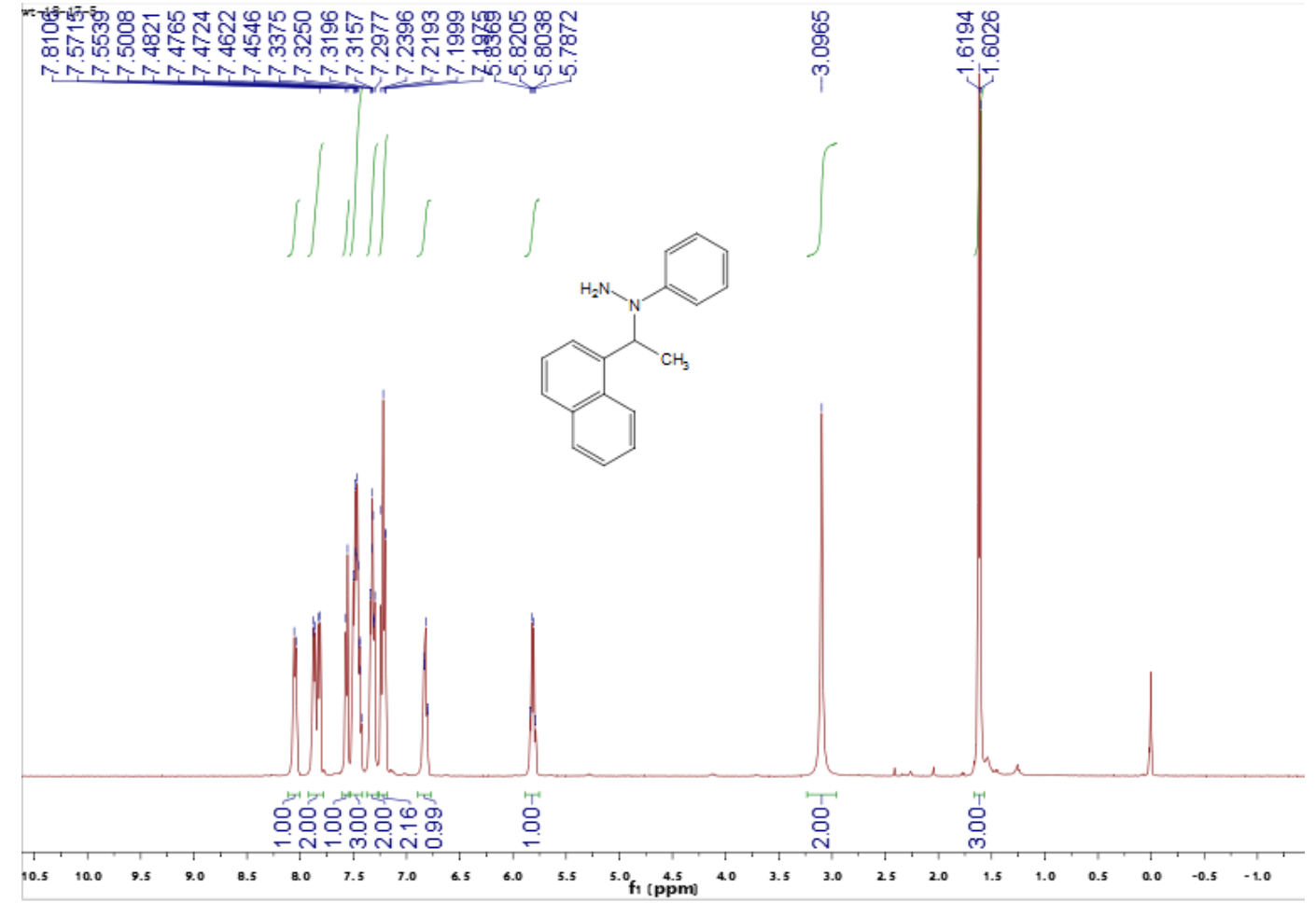

\section{${ }^{13}$ C NMR of 3i}

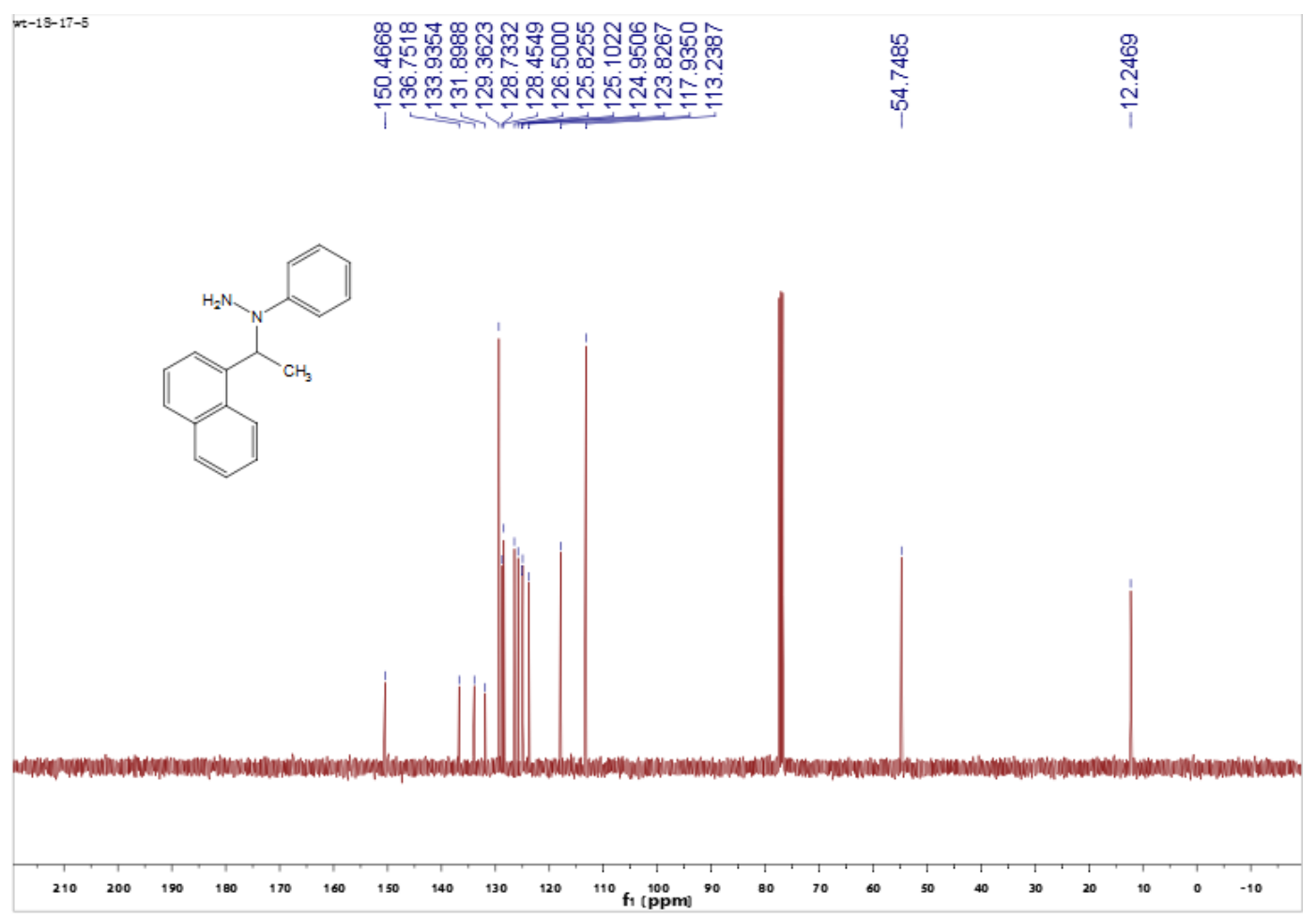


${ }^{1} \mathrm{H}$ NMR of $\mathbf{3 j}$

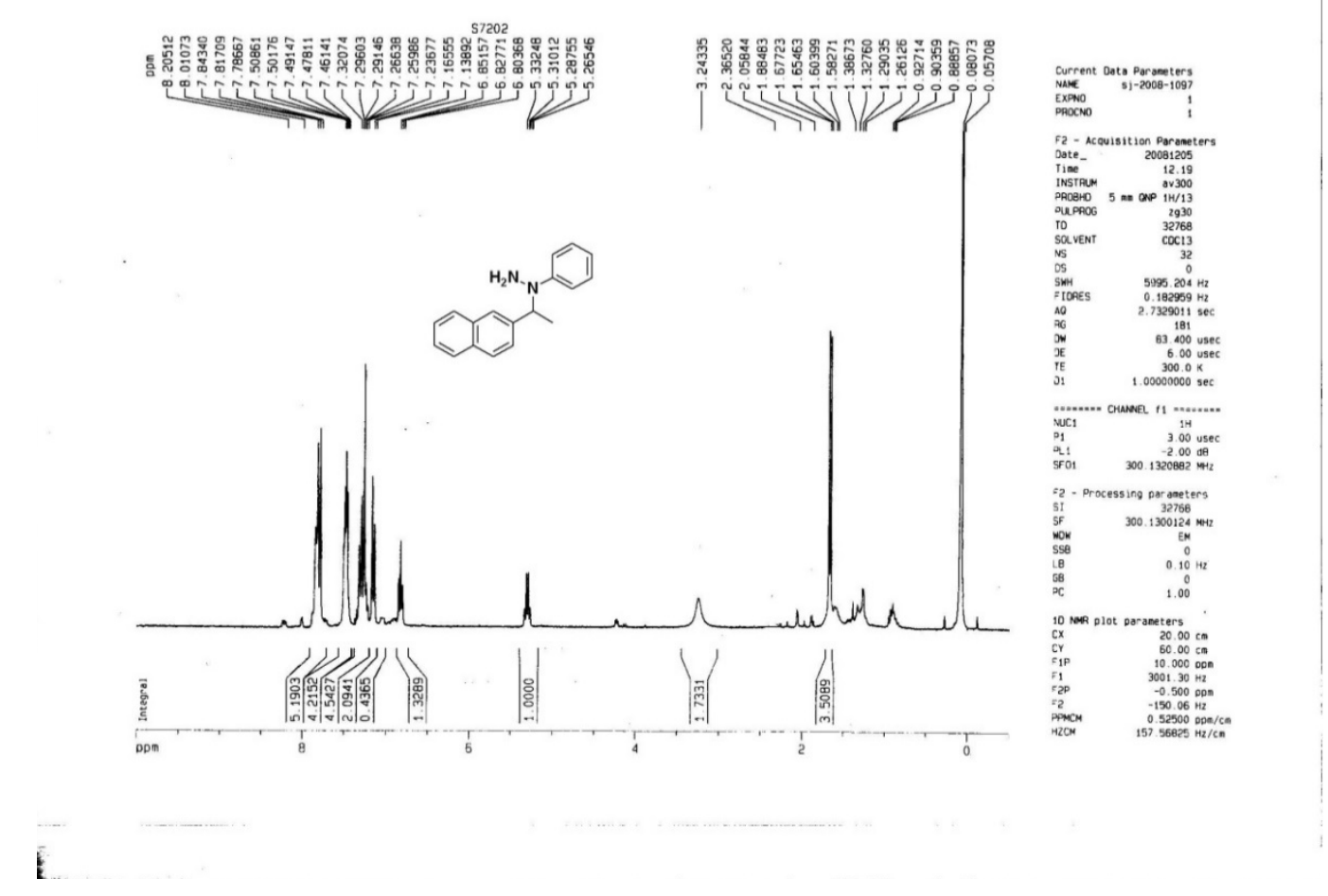

${ }^{13}$ C NMR of $3 j$

Bruker Avance 600 probe: 13C.1H DUL, TE: 300 Ksample: 88252 solvent CDCL3 spectrum: $13 \mathrm{C}$

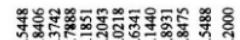

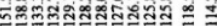

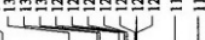

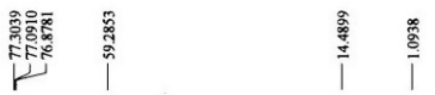
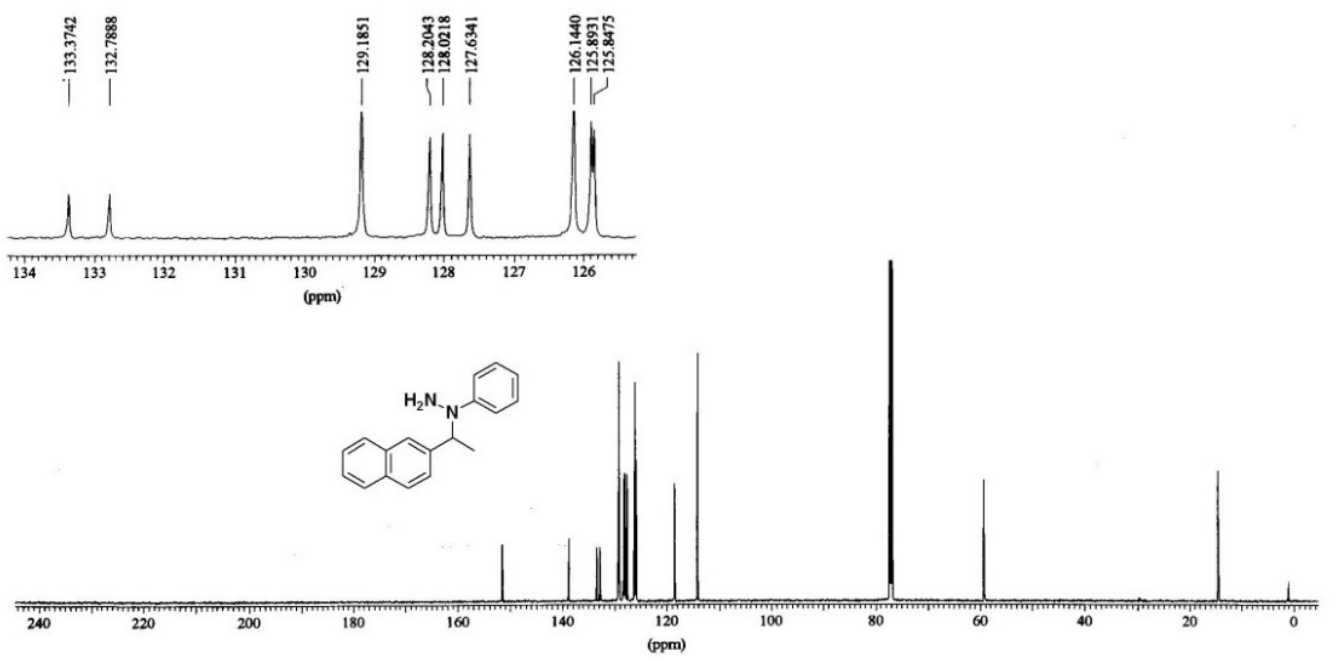
${ }^{1} \mathrm{H}$ NMR of $\mathbf{3 k}$

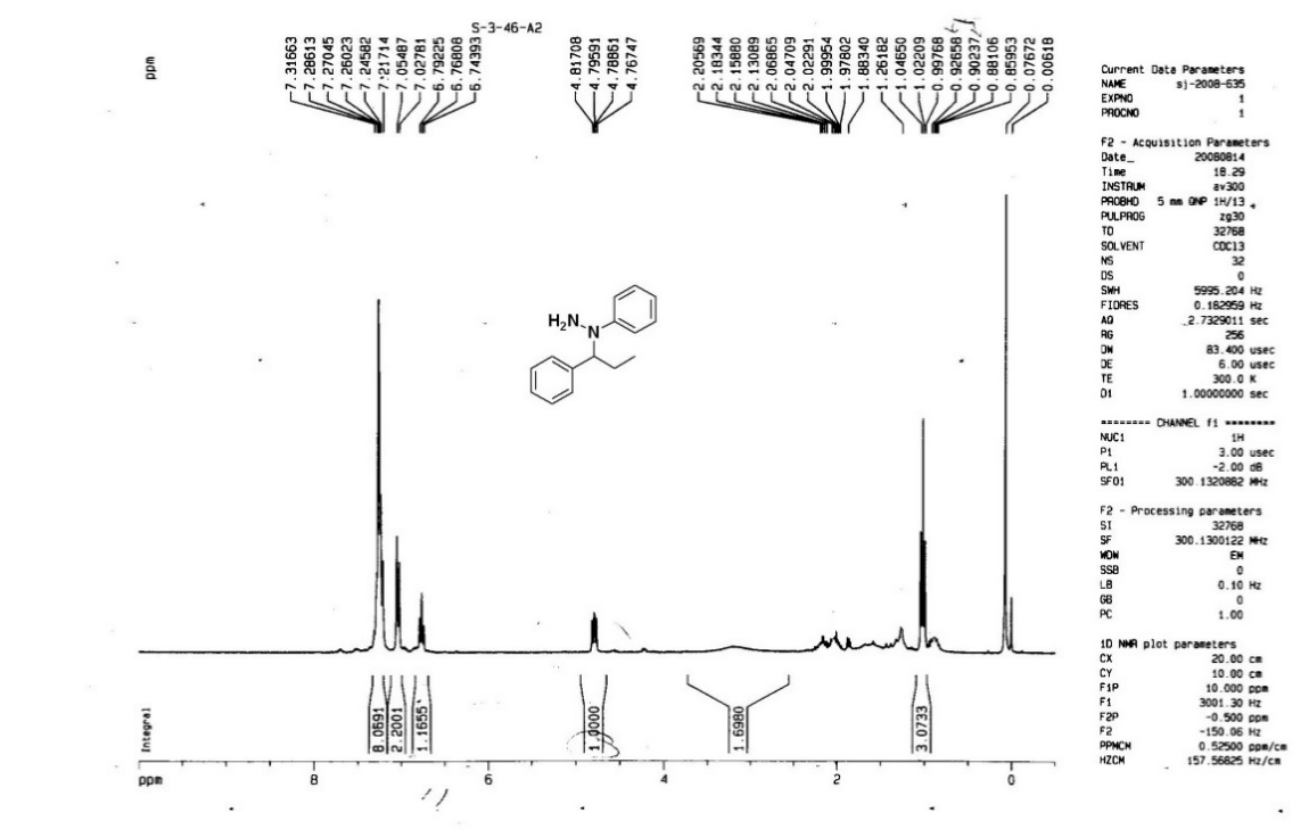

1

${ }^{13}$ C NMR of $3 k$
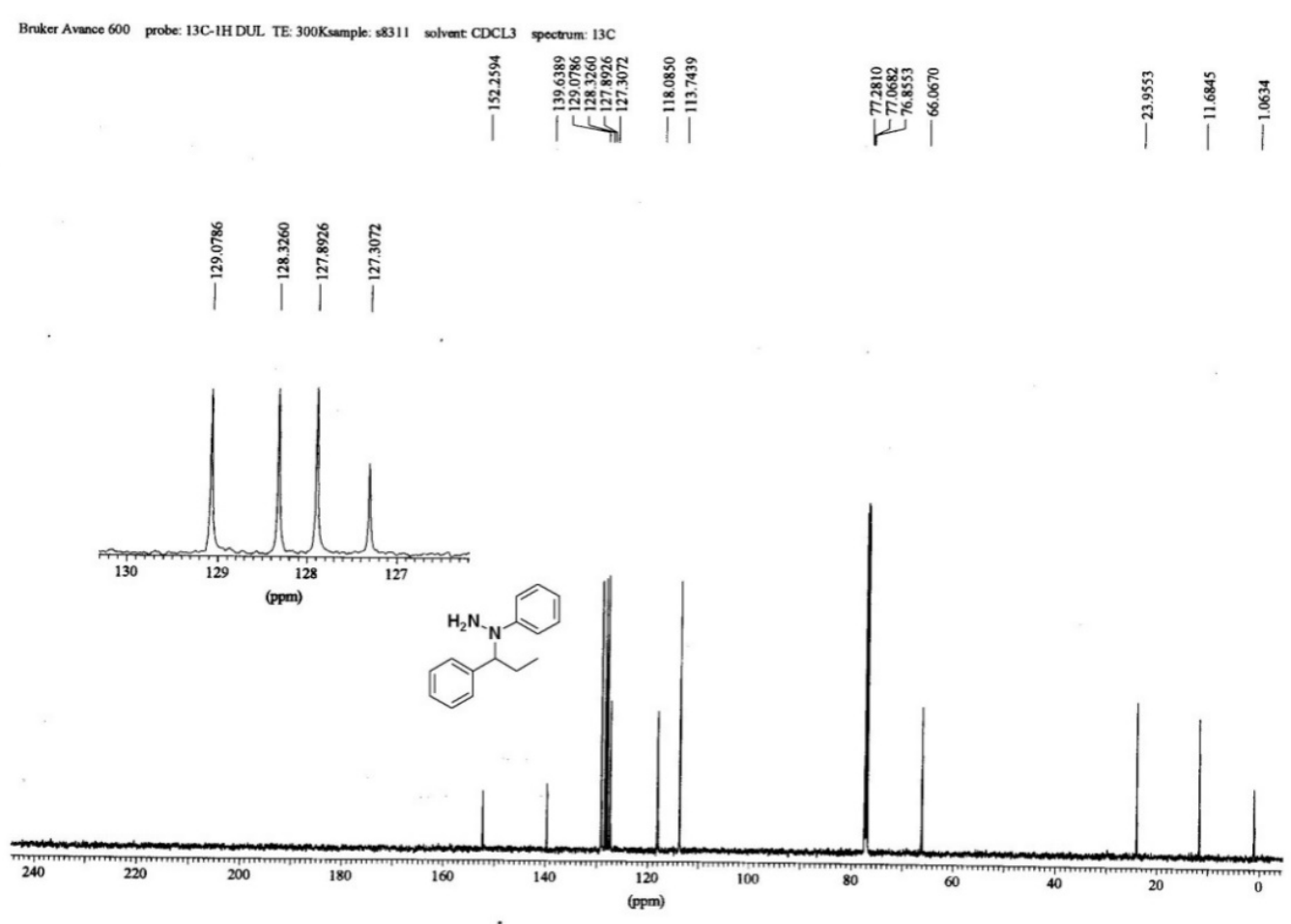
${ }^{1} \mathrm{H}$ NMR of $3 \mathbf{I}$

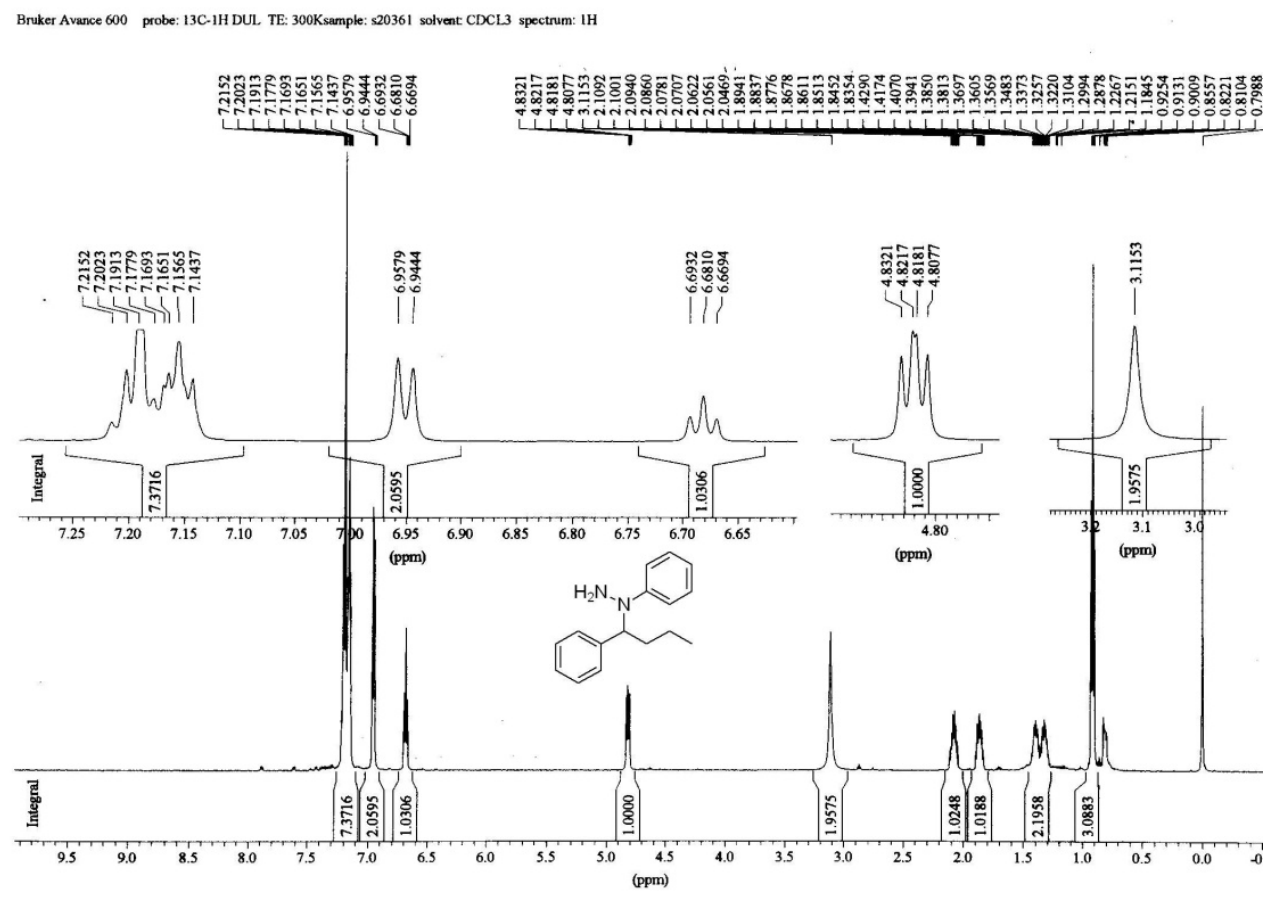

${ }^{13}$ C NMR of 3 I

Bruker Avance 600 probe: $13 \mathrm{C}-1 \mathrm{H}$ DUL, TE: 300Ksample: 203661 solvent: CDCL3 spectrum: $13 \mathrm{C}$

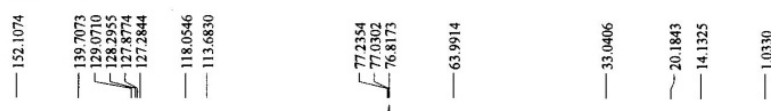

|

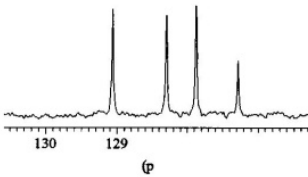

$\mathrm{H}_{2} \mathrm{~N}_{-\mathrm{N}}$

1)

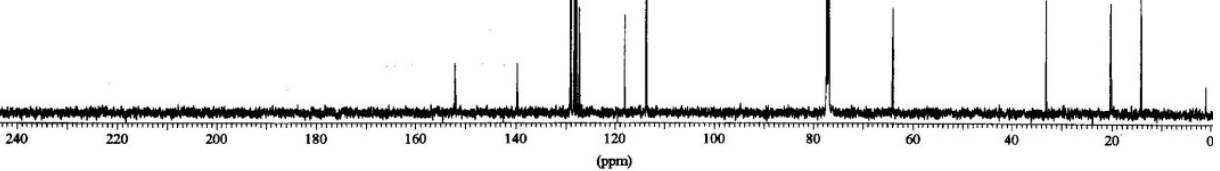


${ }^{1} \mathrm{H}$ NMR of $\mathbf{3 m}$

wt-18-17-6

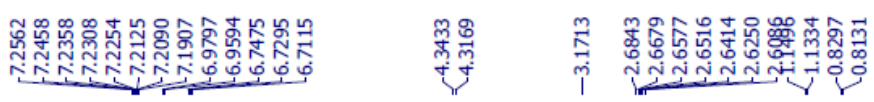

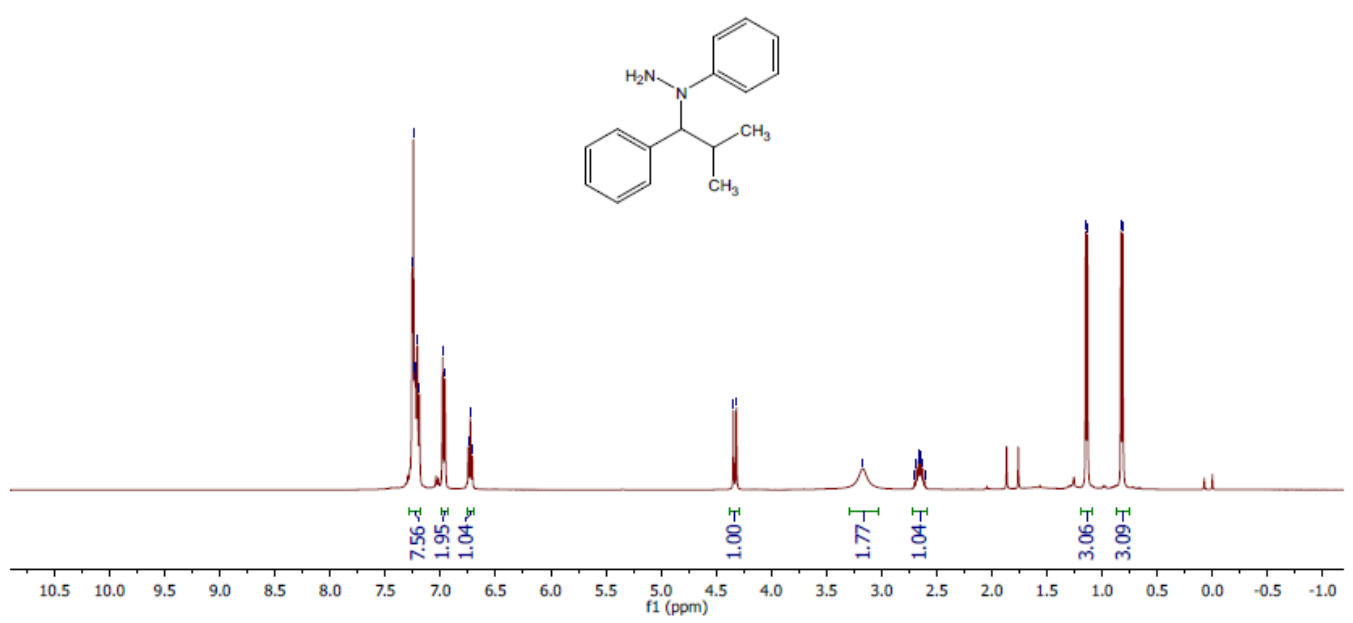

${ }^{13} \mathrm{C}$ NMR of $3 \mathrm{~m}$

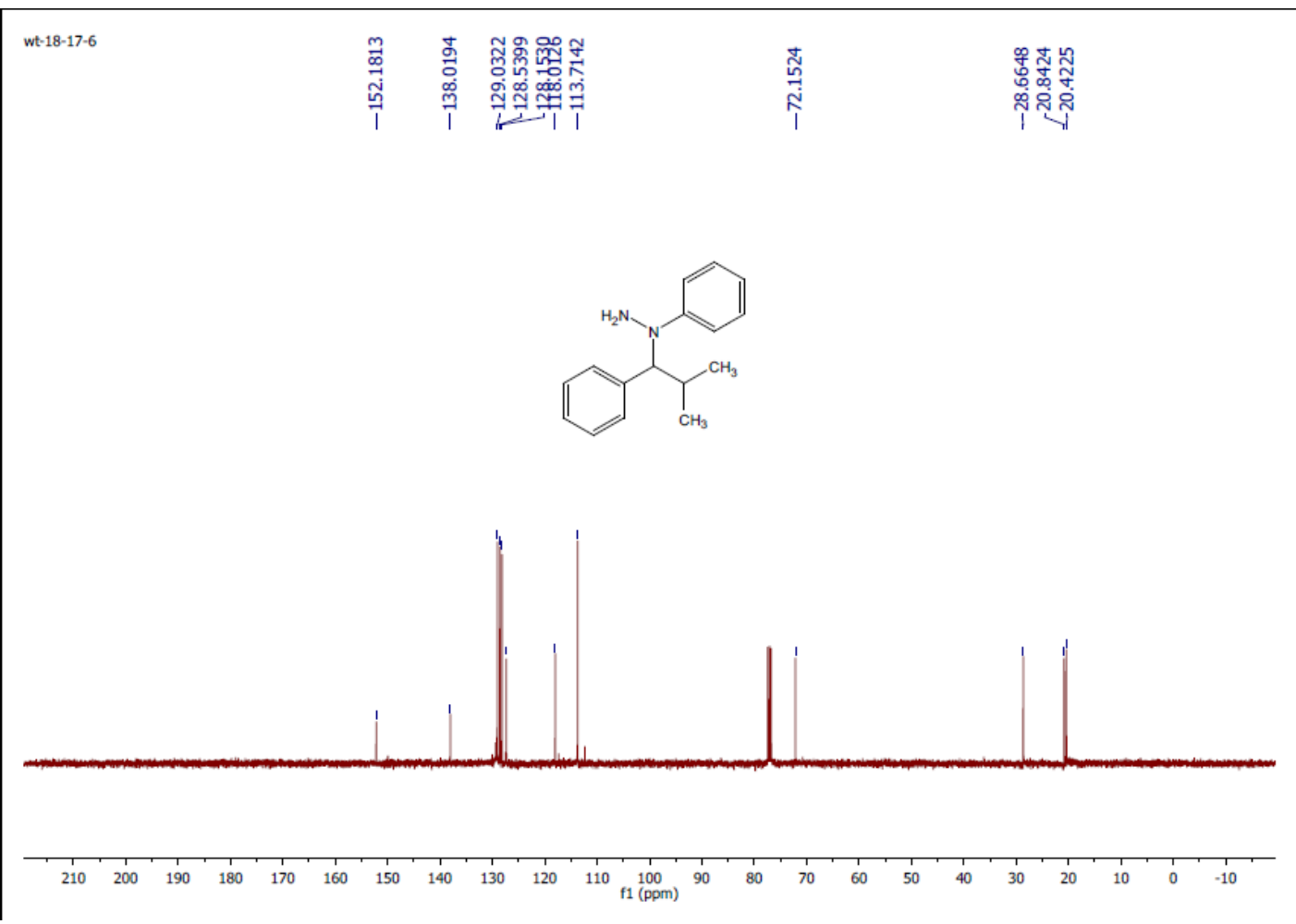


${ }^{1} \mathrm{H}$ NMR of $\mathbf{3 n}$

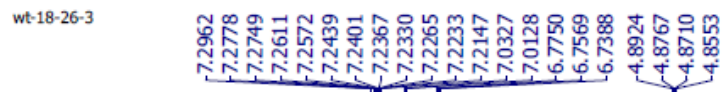

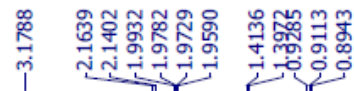

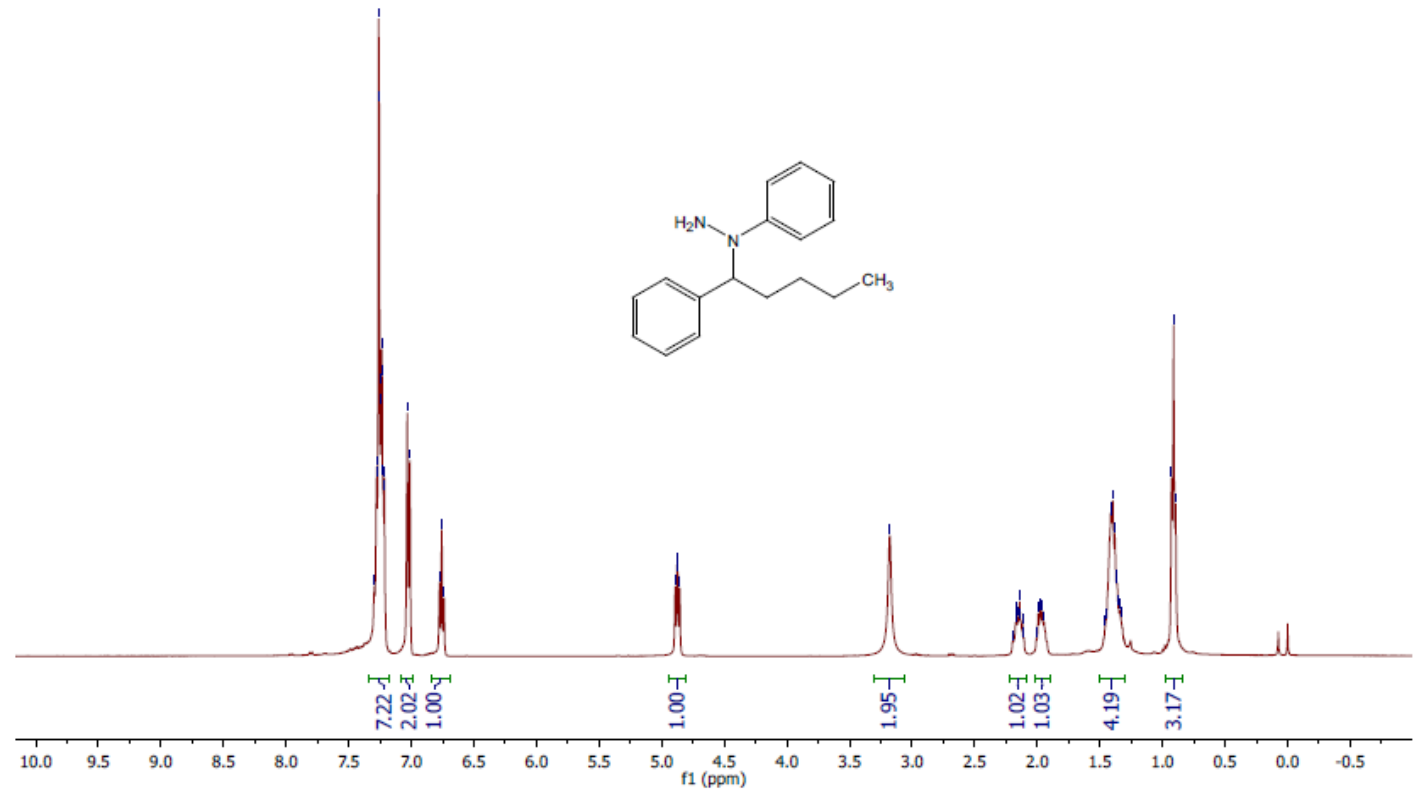

${ }^{13}$ C NMR of $3 n$

wt:18-26-3

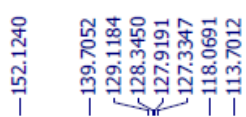

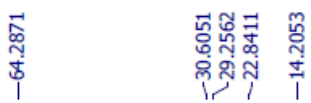

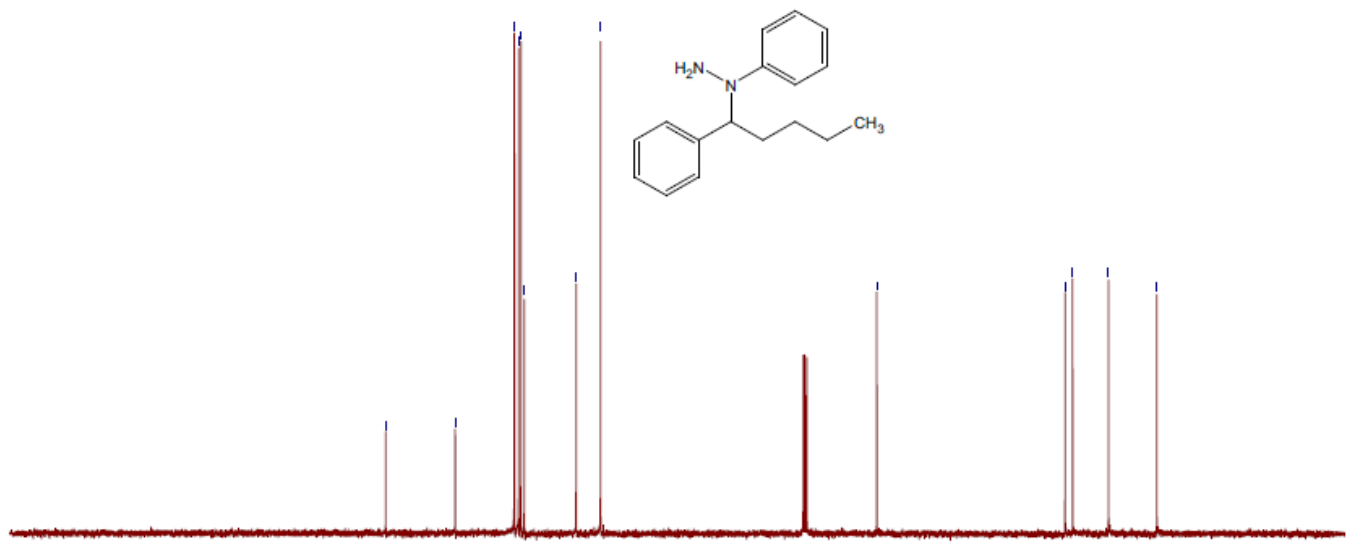

$\begin{array}{lllllllllllllllllllllllll}210 & 200 & 190 & 180 & 170 & 160 & 150 & 140 & 130 & 120 & 110 & 100 & 90 & 80 & 70 & 60 & 50 & 40 & 30 & 20 & 10 & 0 & -10\end{array}$ 
${ }^{1} \mathrm{H}$ NMR of $\mathbf{3 o}$

wt-18-42-1
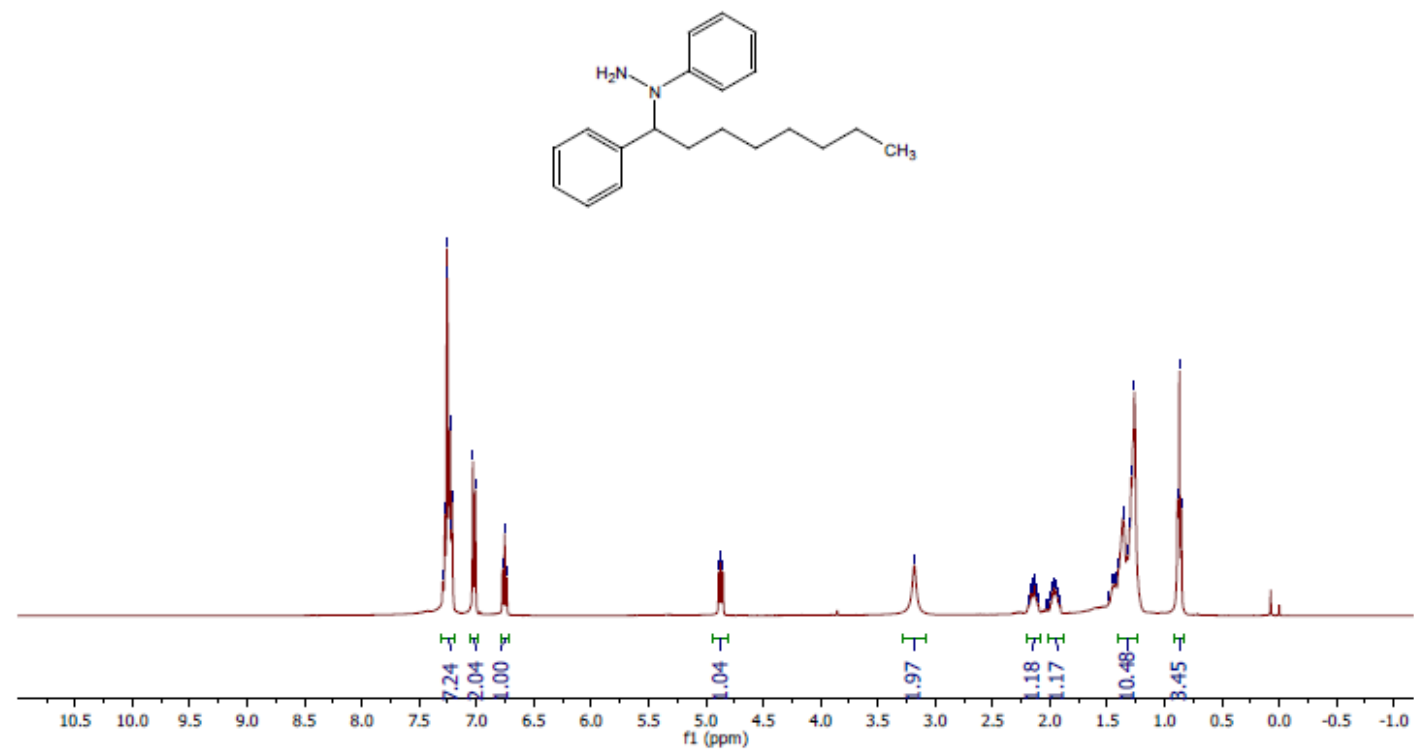

\section{${ }^{13}$ C NMR of 30}

wt-18-42-1
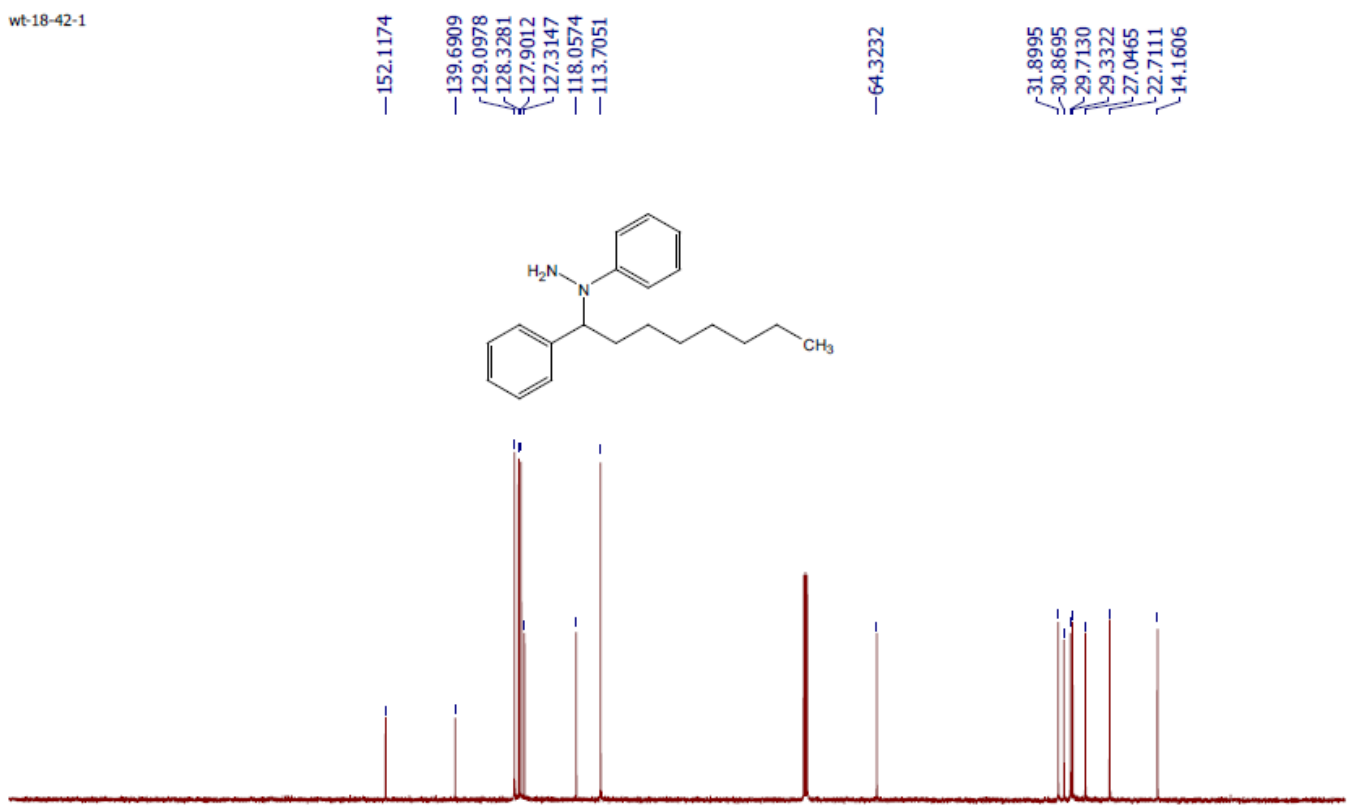

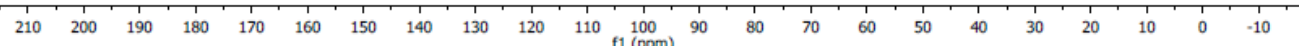


${ }^{1} \mathrm{H}$ NMR of $\mathbf{3 p}$

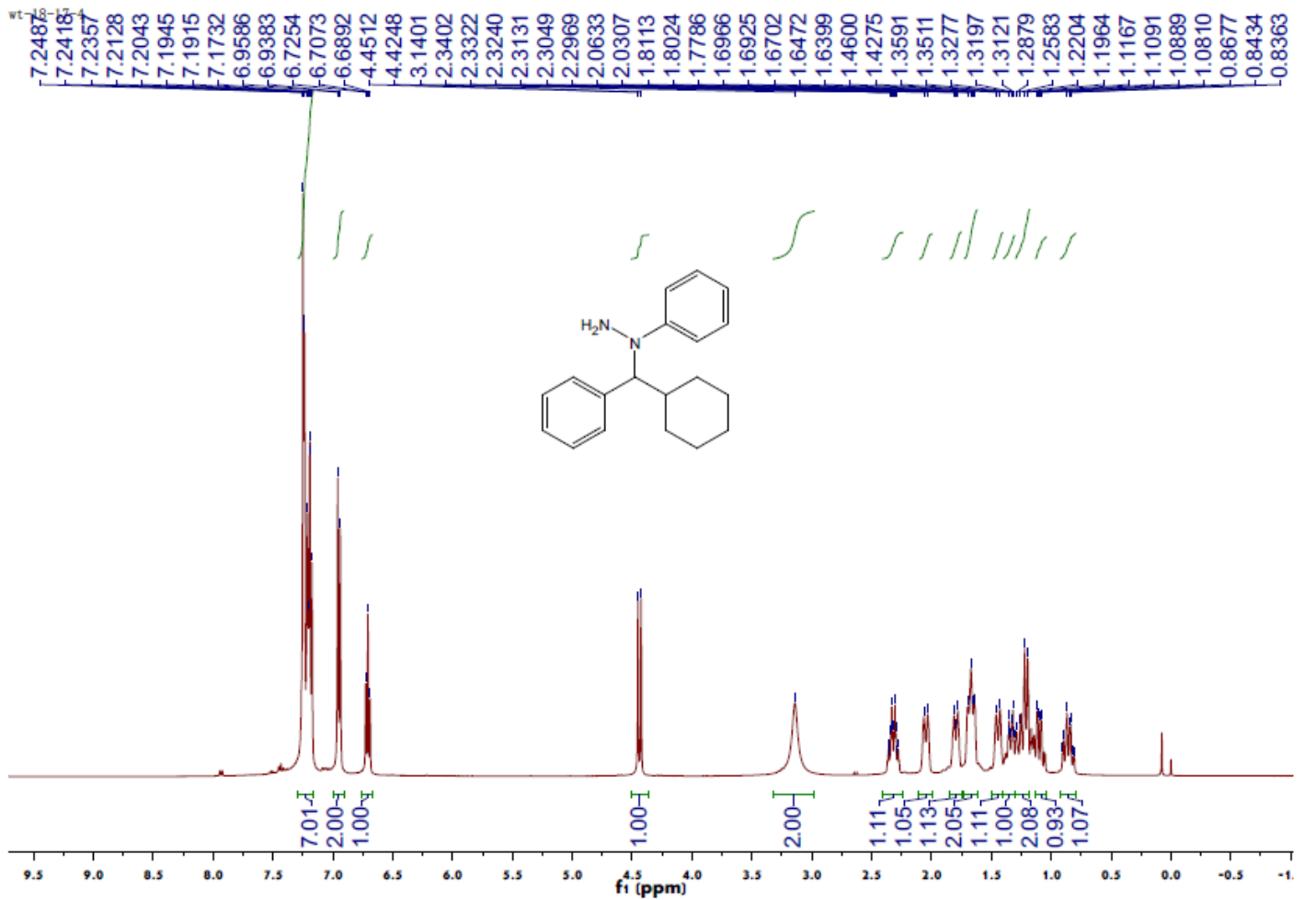

${ }^{13}$ C NMR of 3p

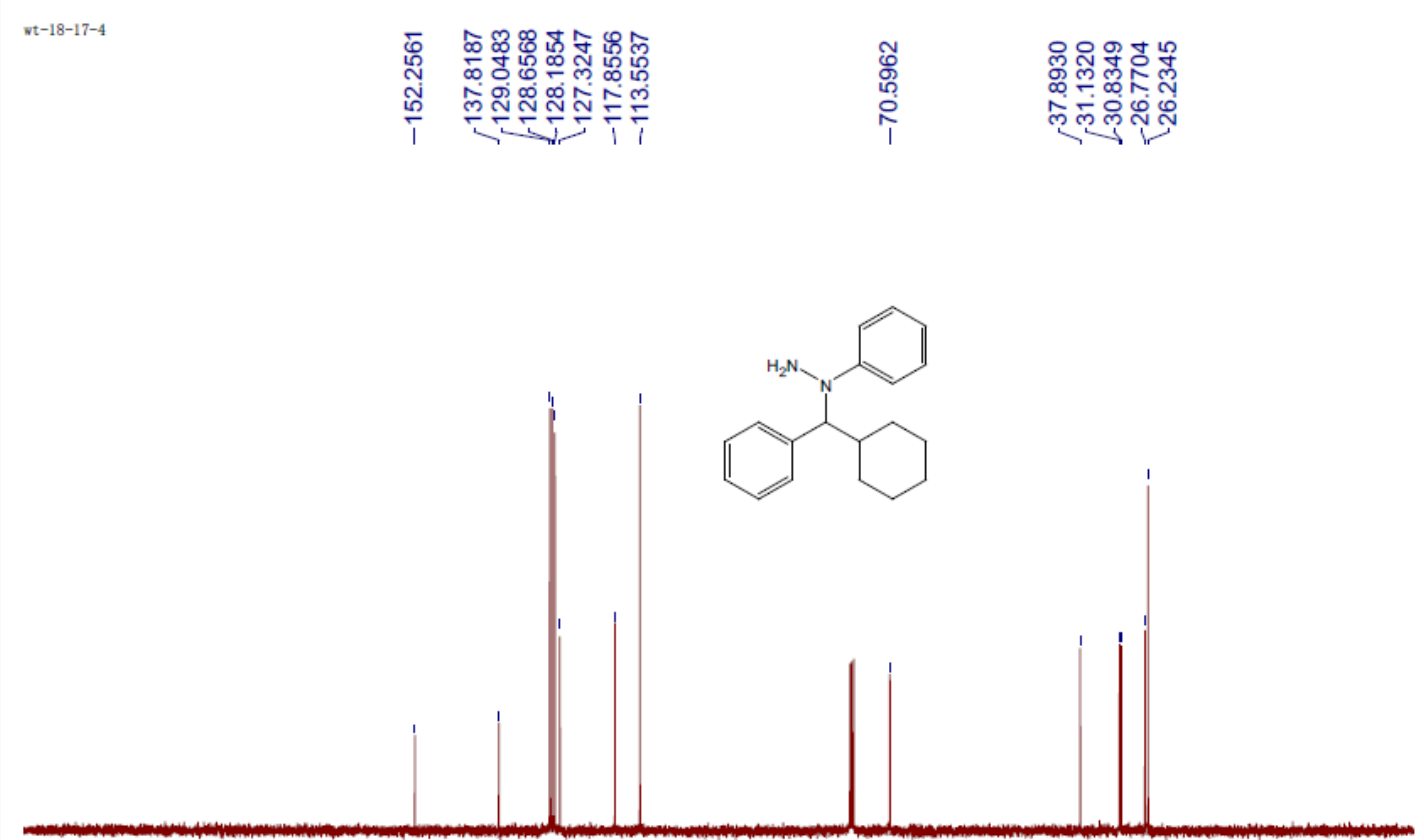

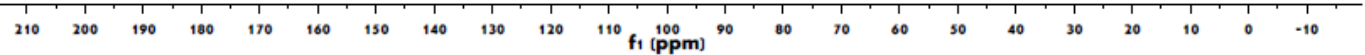


${ }^{1} \mathrm{H}$ NMR of $\mathbf{3 q}$

wt-18-26-2

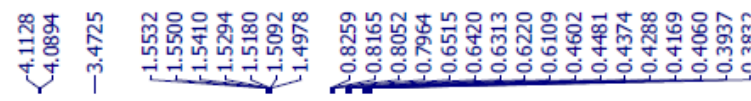

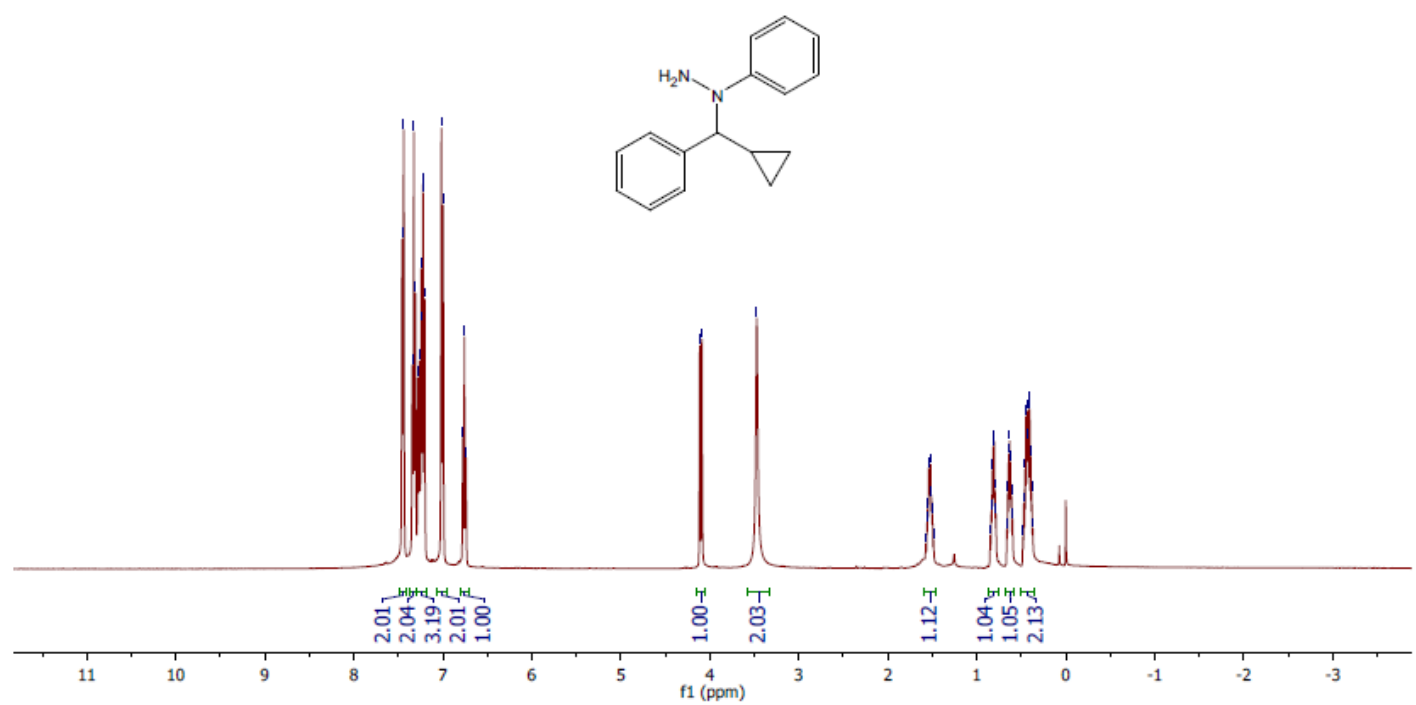

${ }^{13}$ C NMR of $3 q$

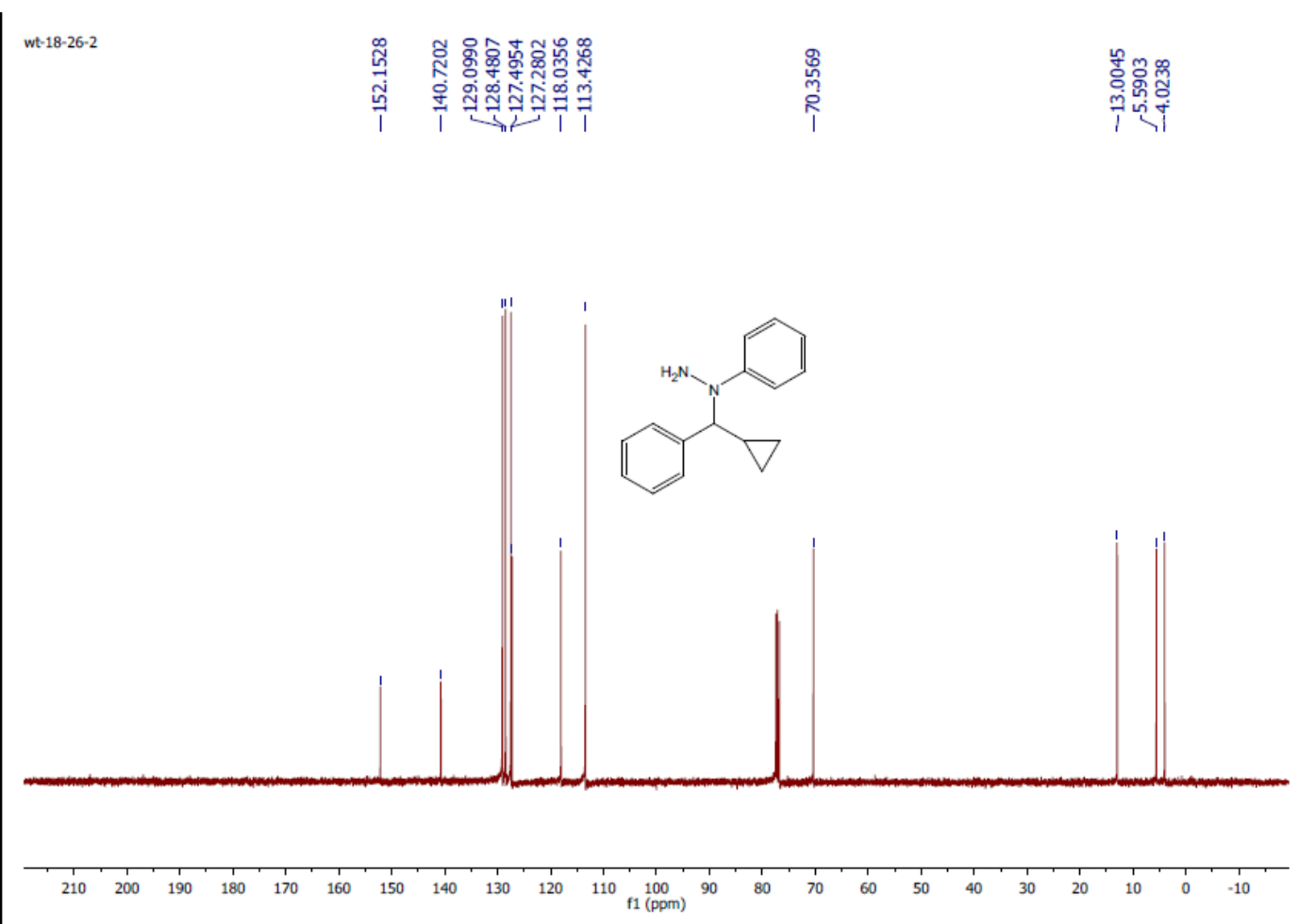




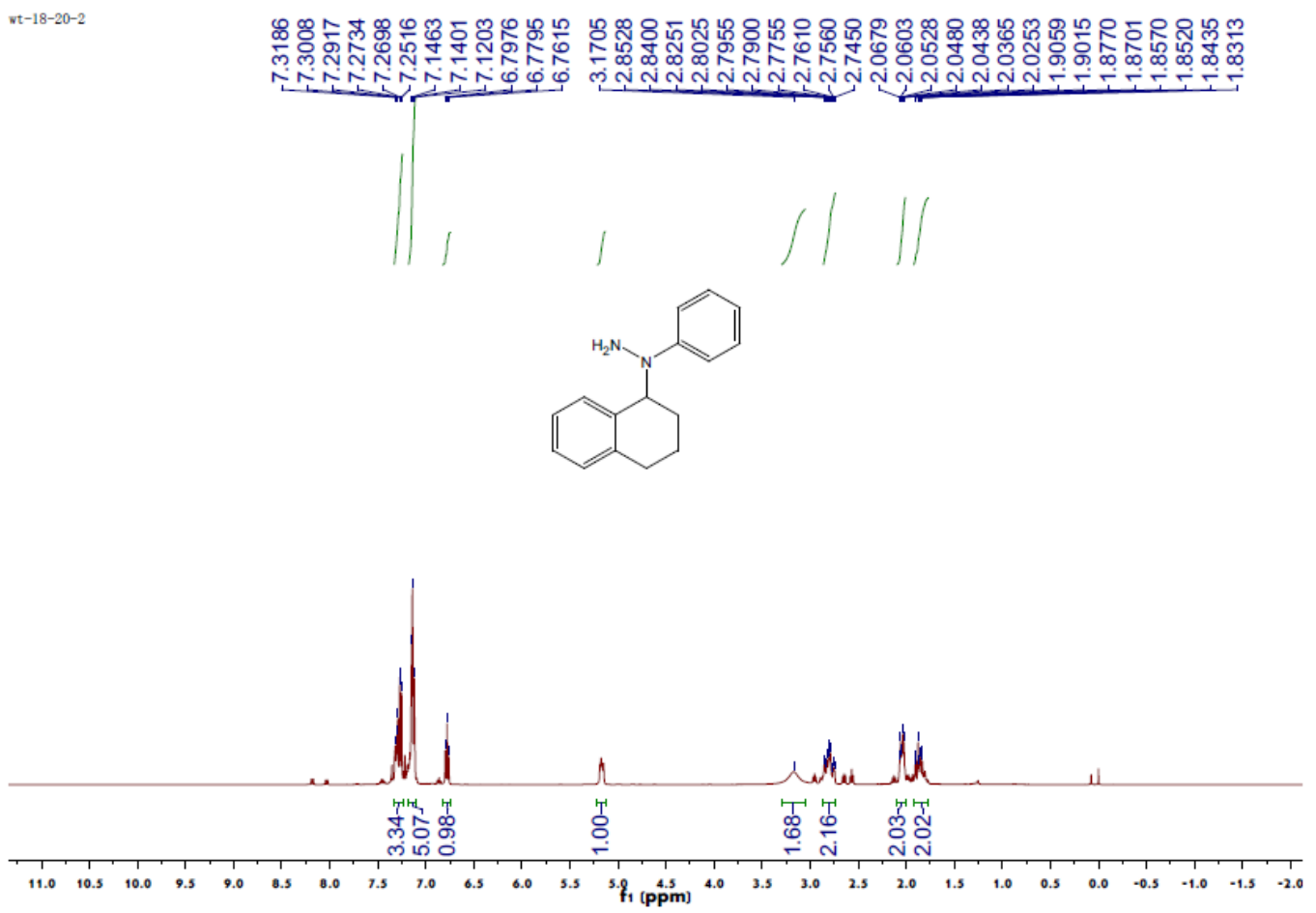

${ }^{13}$ C NMR of 3r

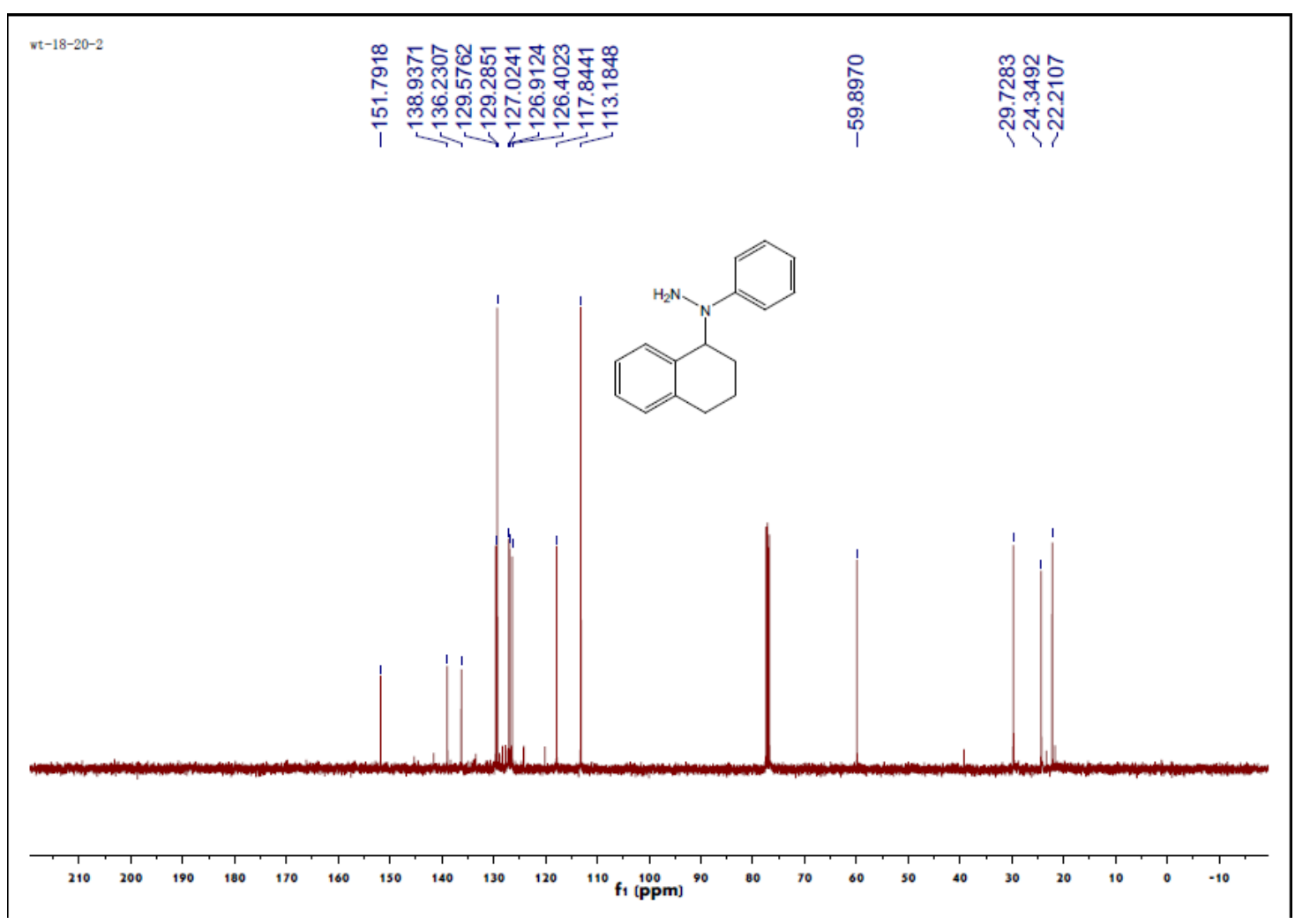


${ }^{1} \mathrm{H}$ NMR of $3 \mathbf{s}$

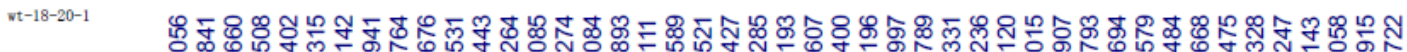

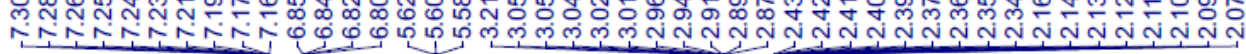

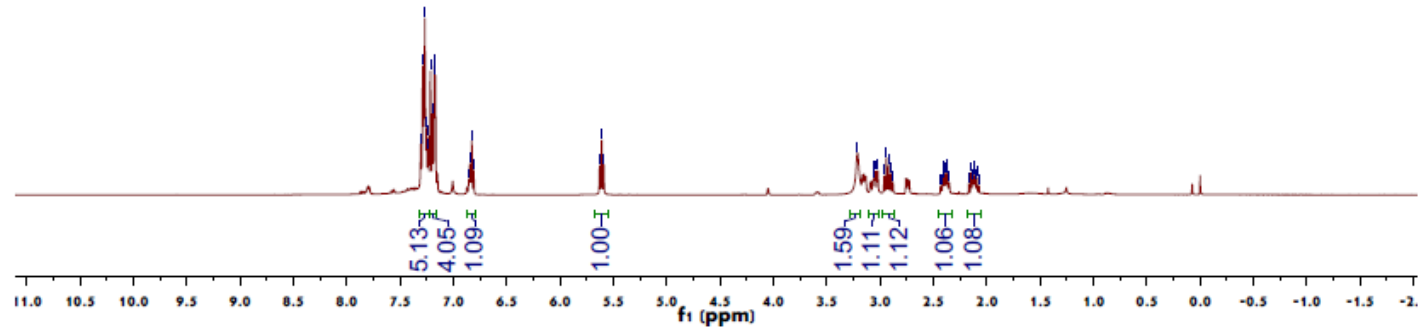

${ }^{13}$ C NMR of $3 \mathrm{~s}$

vt-18-20-2

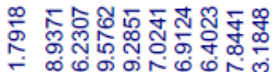

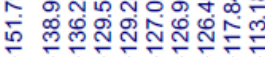

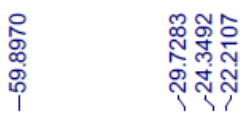

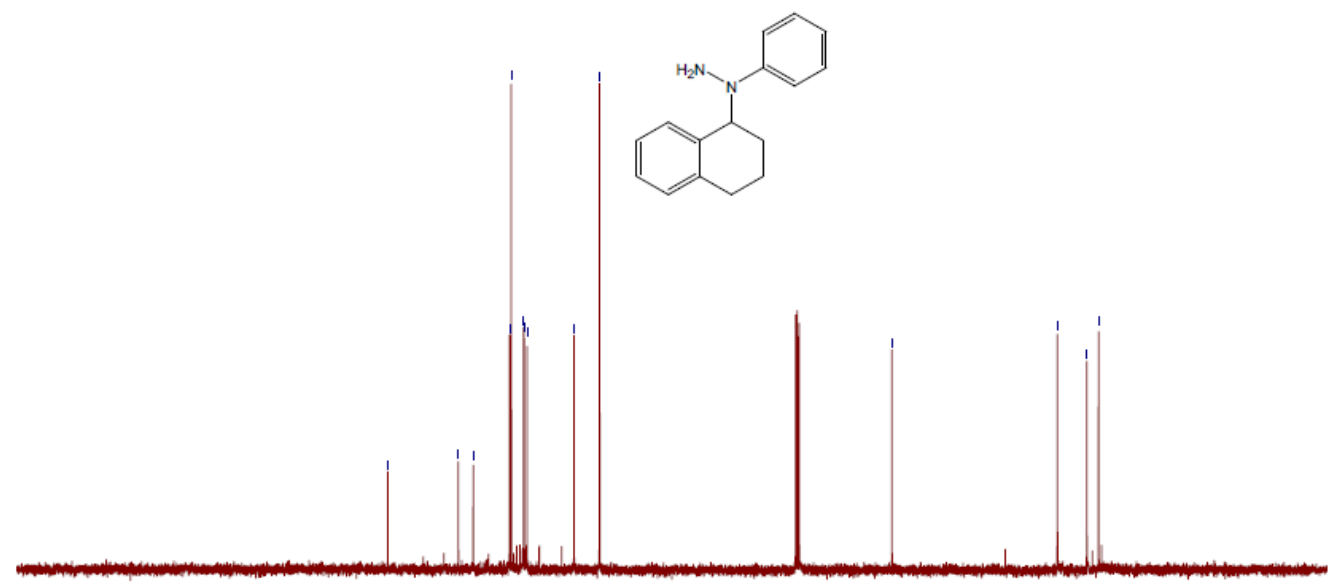

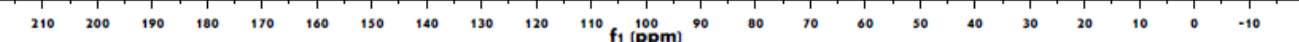


${ }^{1} \mathrm{H}$ NMR of $\mathbf{3 t}$

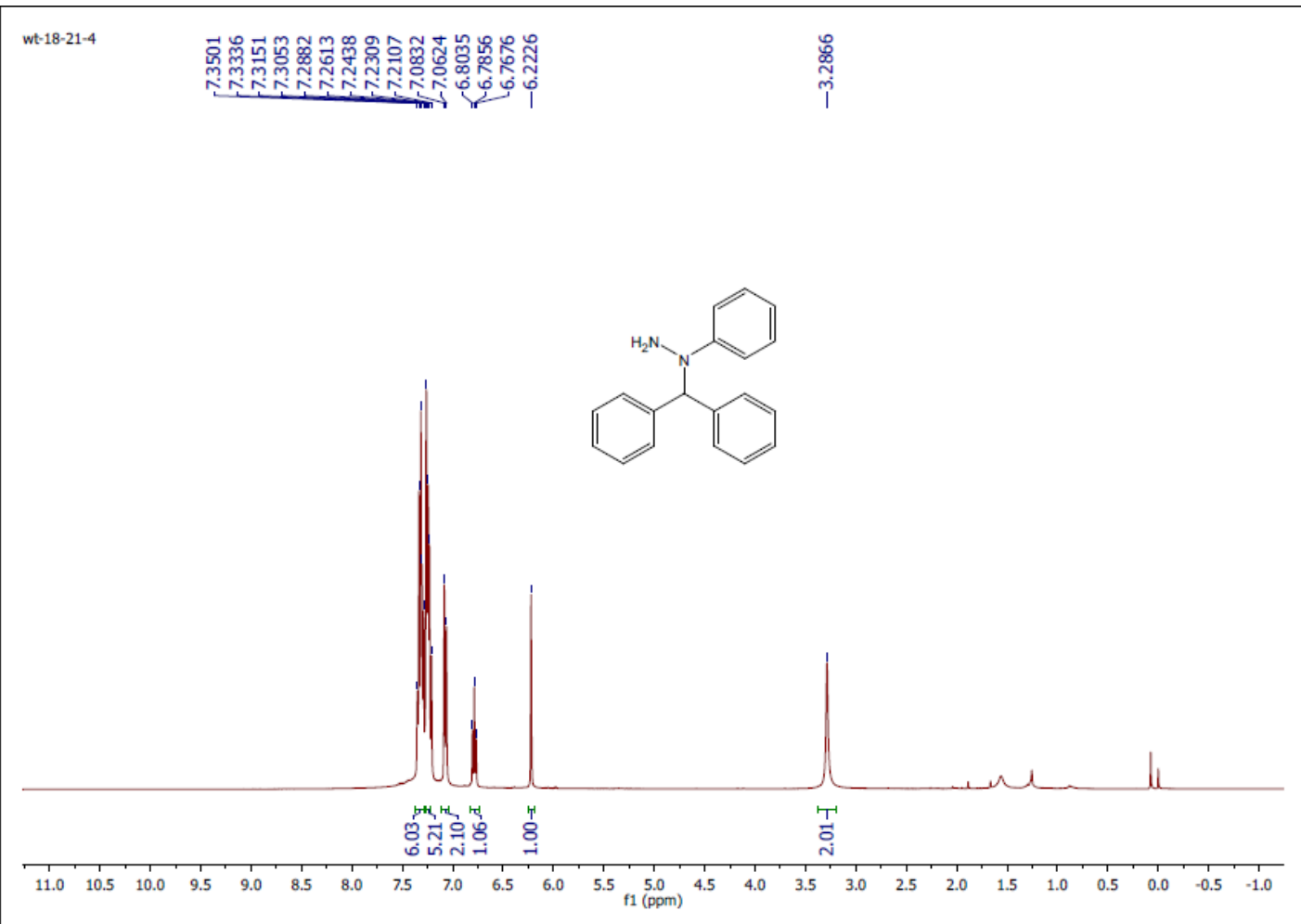

${ }^{13}$ C NMR of $3 t$

vt-18-21-4
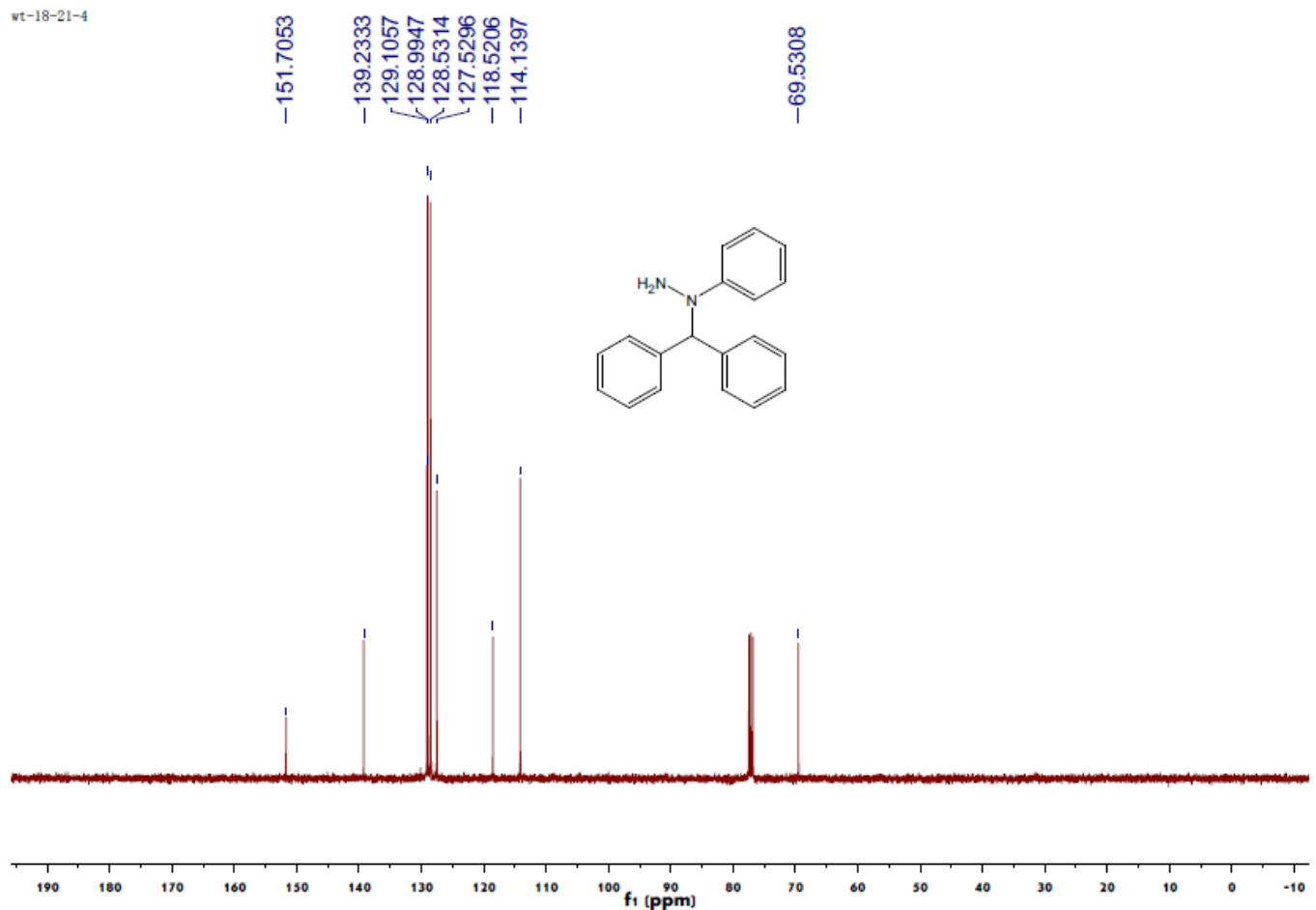
${ }^{1} \mathrm{H}$ NMR of $\mathbf{3 u}$

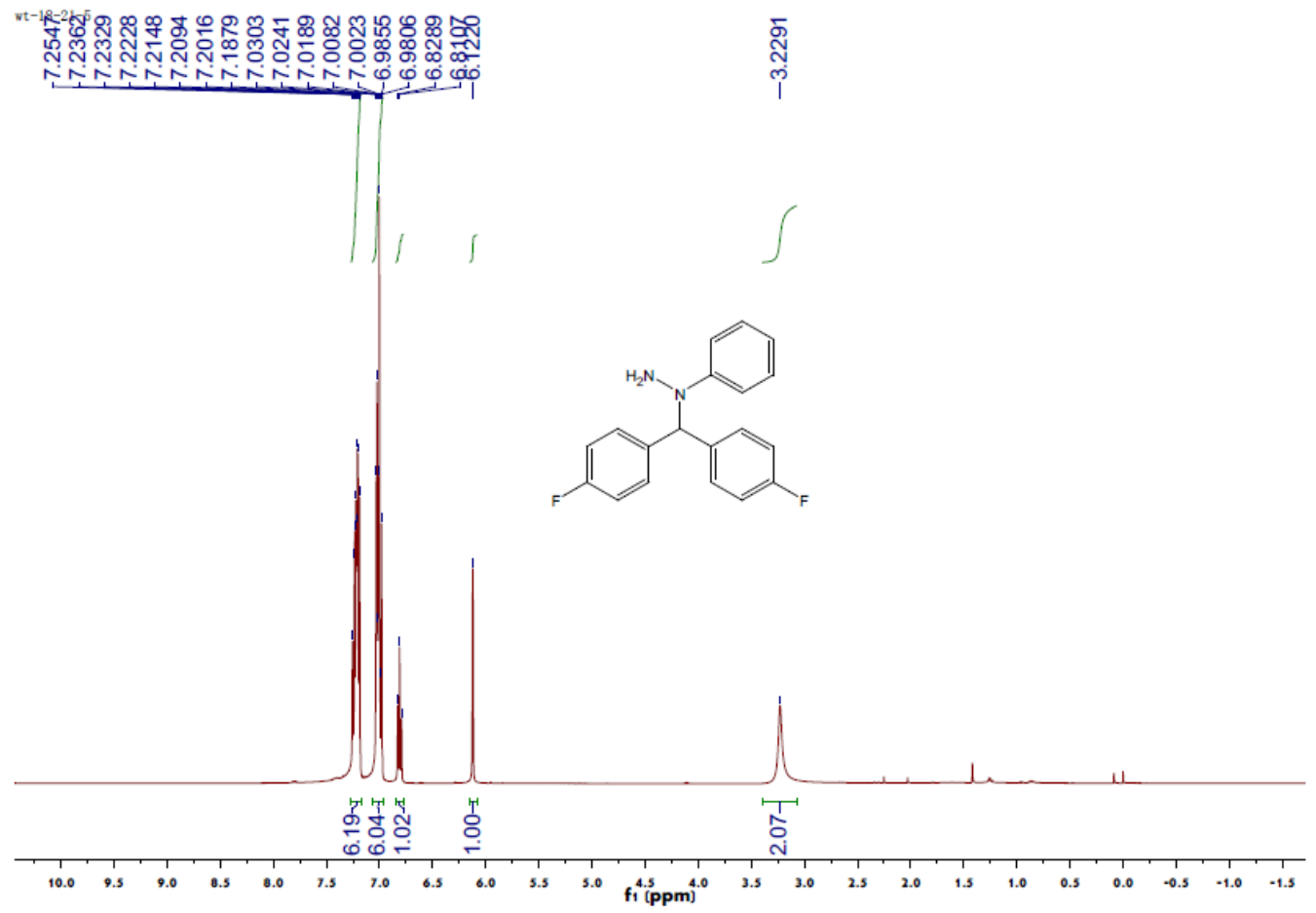

${ }^{13}$ C NMR of $3 u$

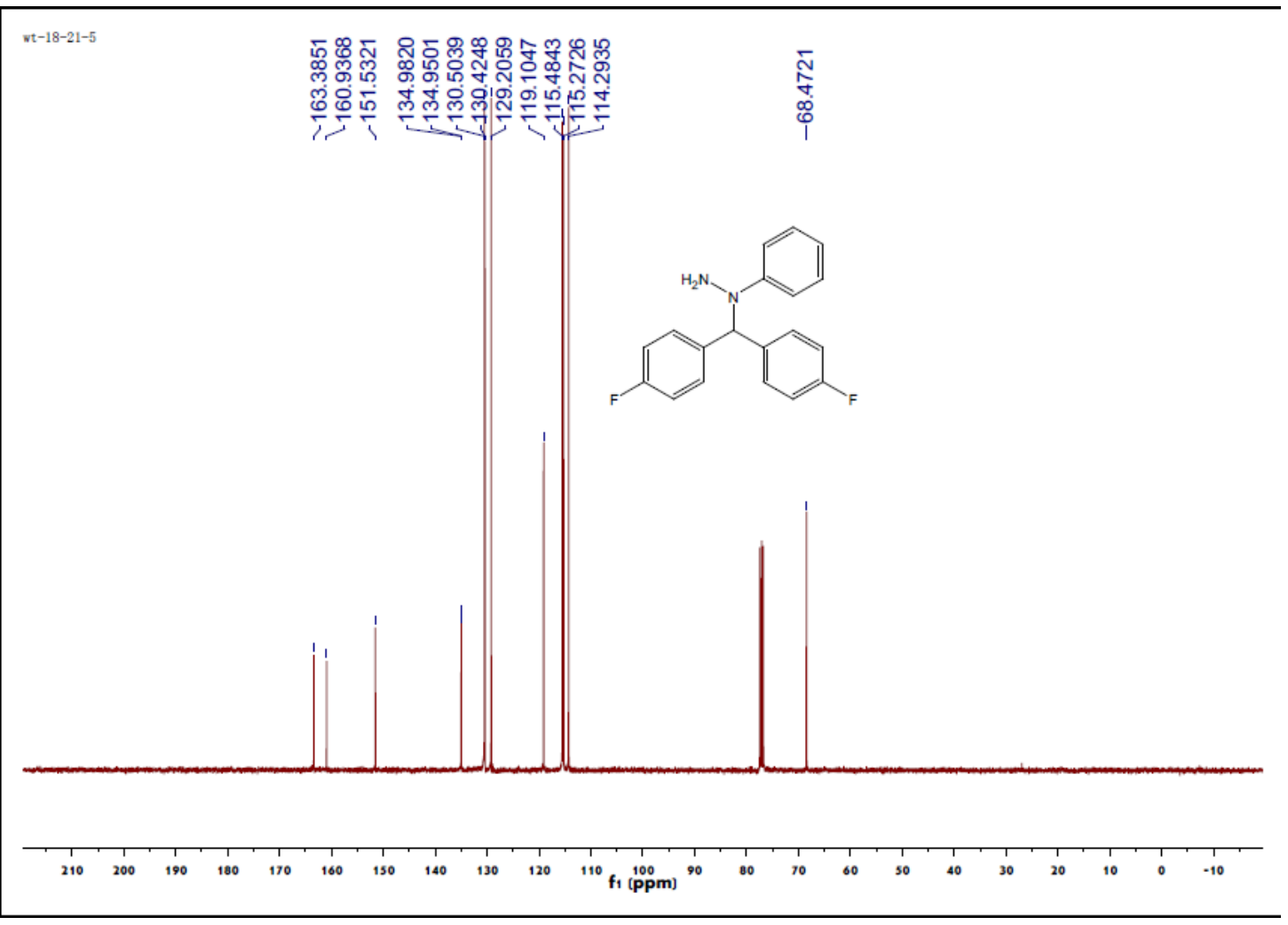


${ }^{1} \mathrm{H}$ NMR of $\mathbf{3 v}$

wt-18-21-6
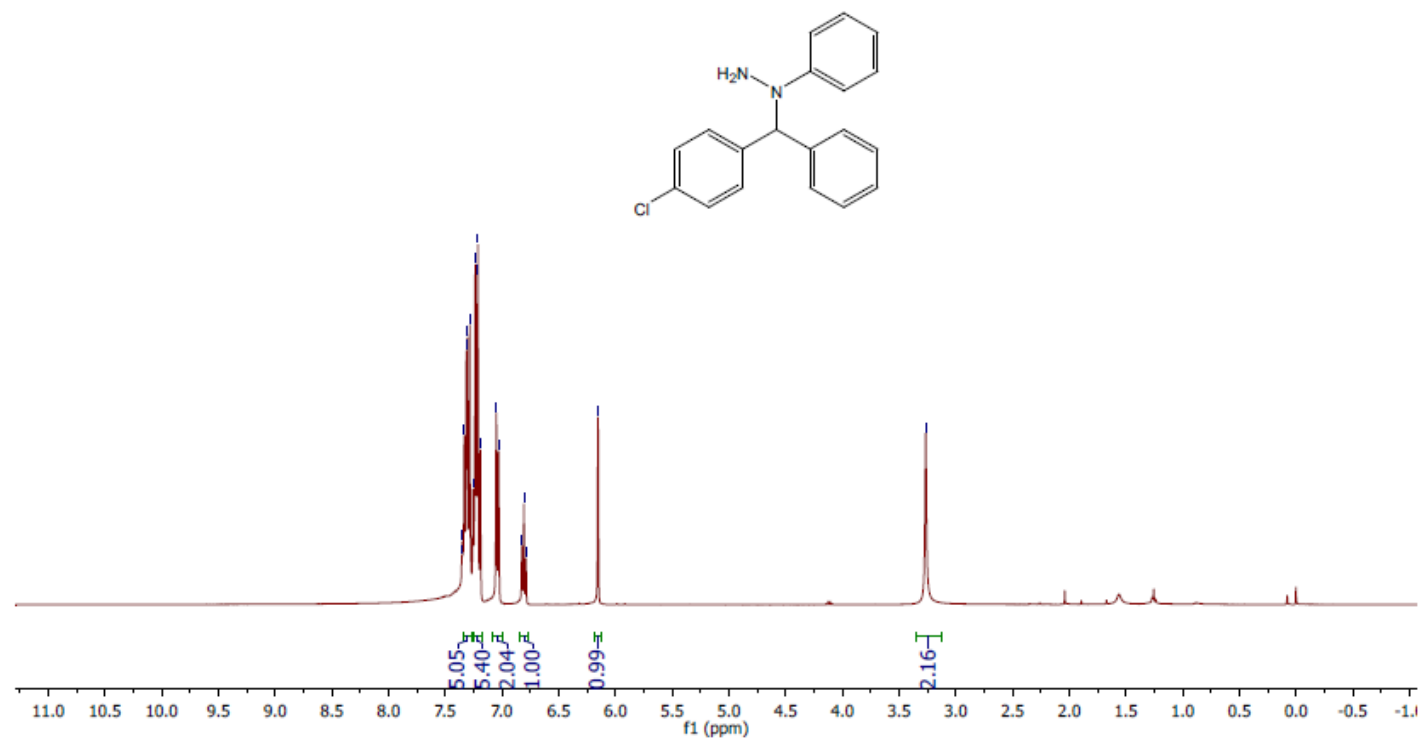

${ }^{13}$ C NMR of 3v

\begin{tabular}{|c|c|}
\hline $\mathrm{vt}-18-21-6$ & 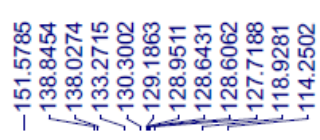 \\
\hline
\end{tabular}
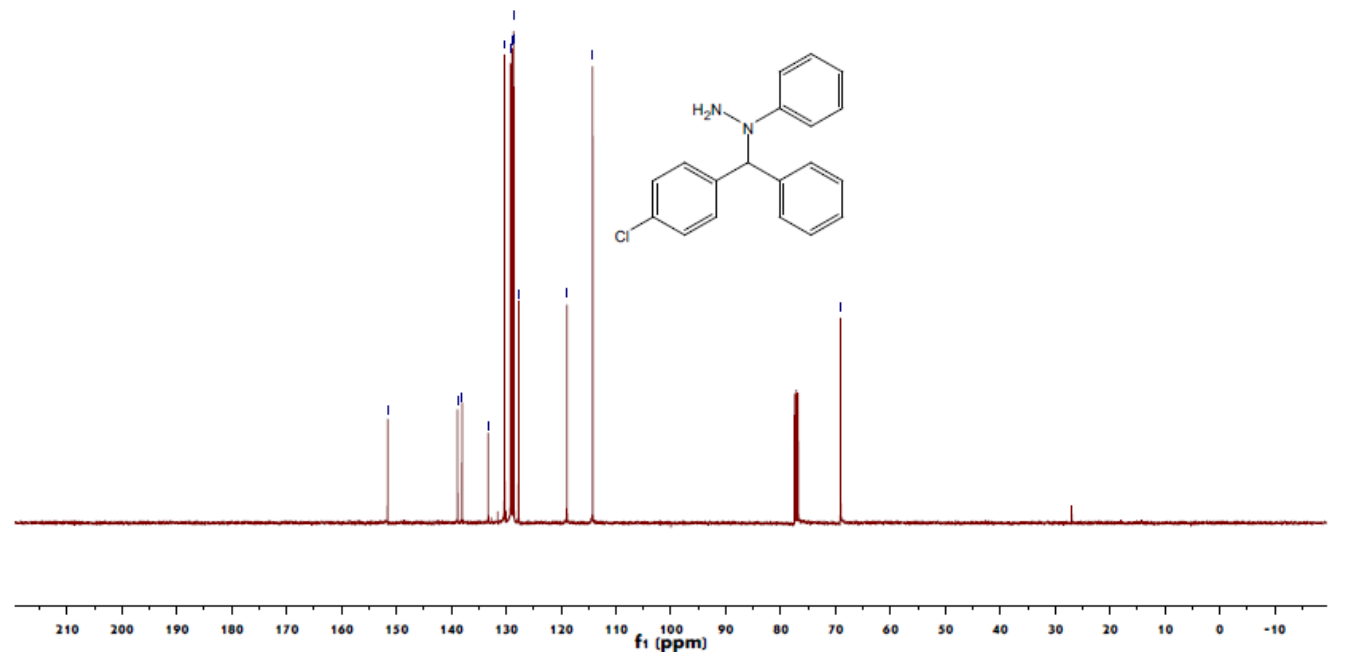
${ }^{1} \mathrm{H}$ NMR of $\mathbf{3 w}$

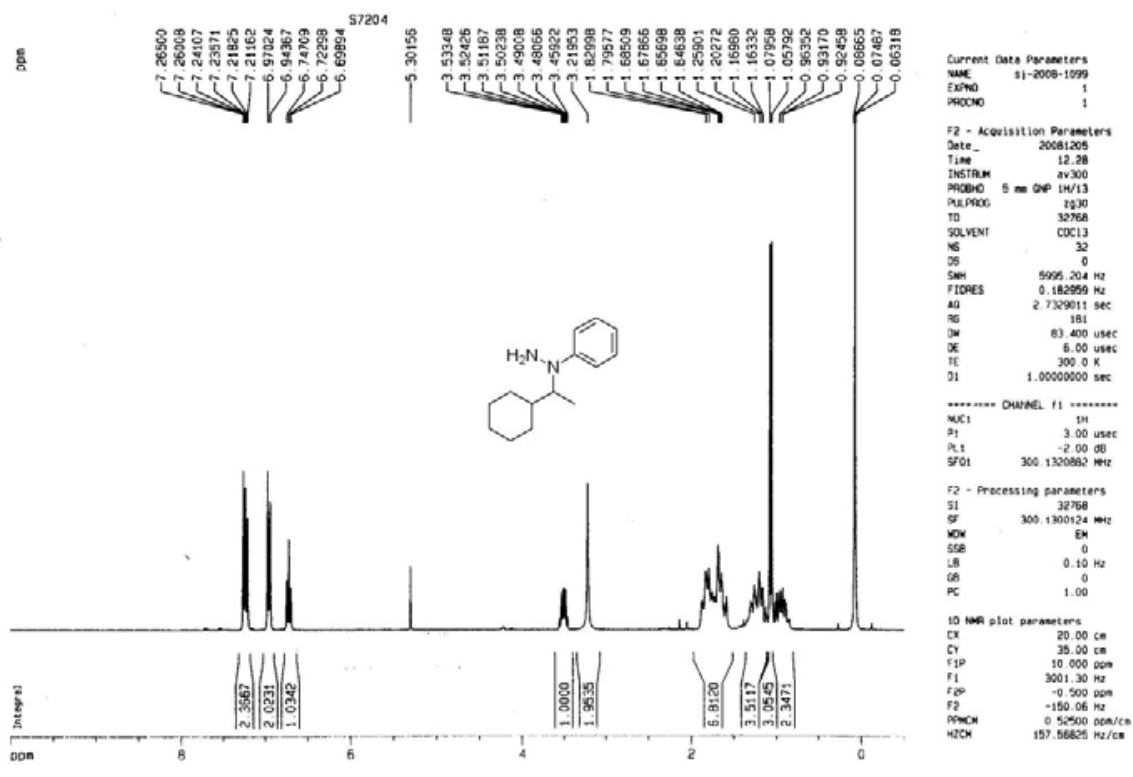

${ }^{13}$ C NMR of $3 w$

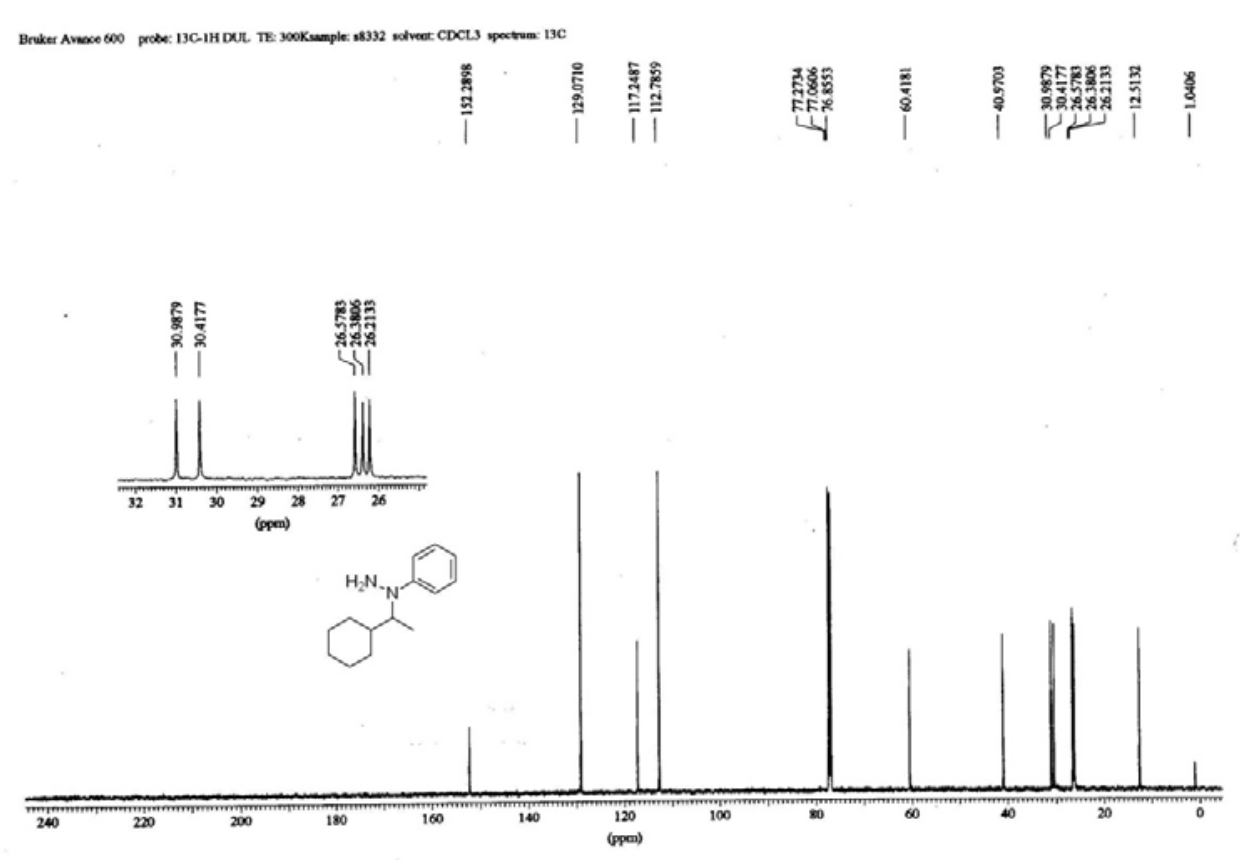


${ }^{1} \mathrm{H}$ NMR of $\mathbf{3 x}$

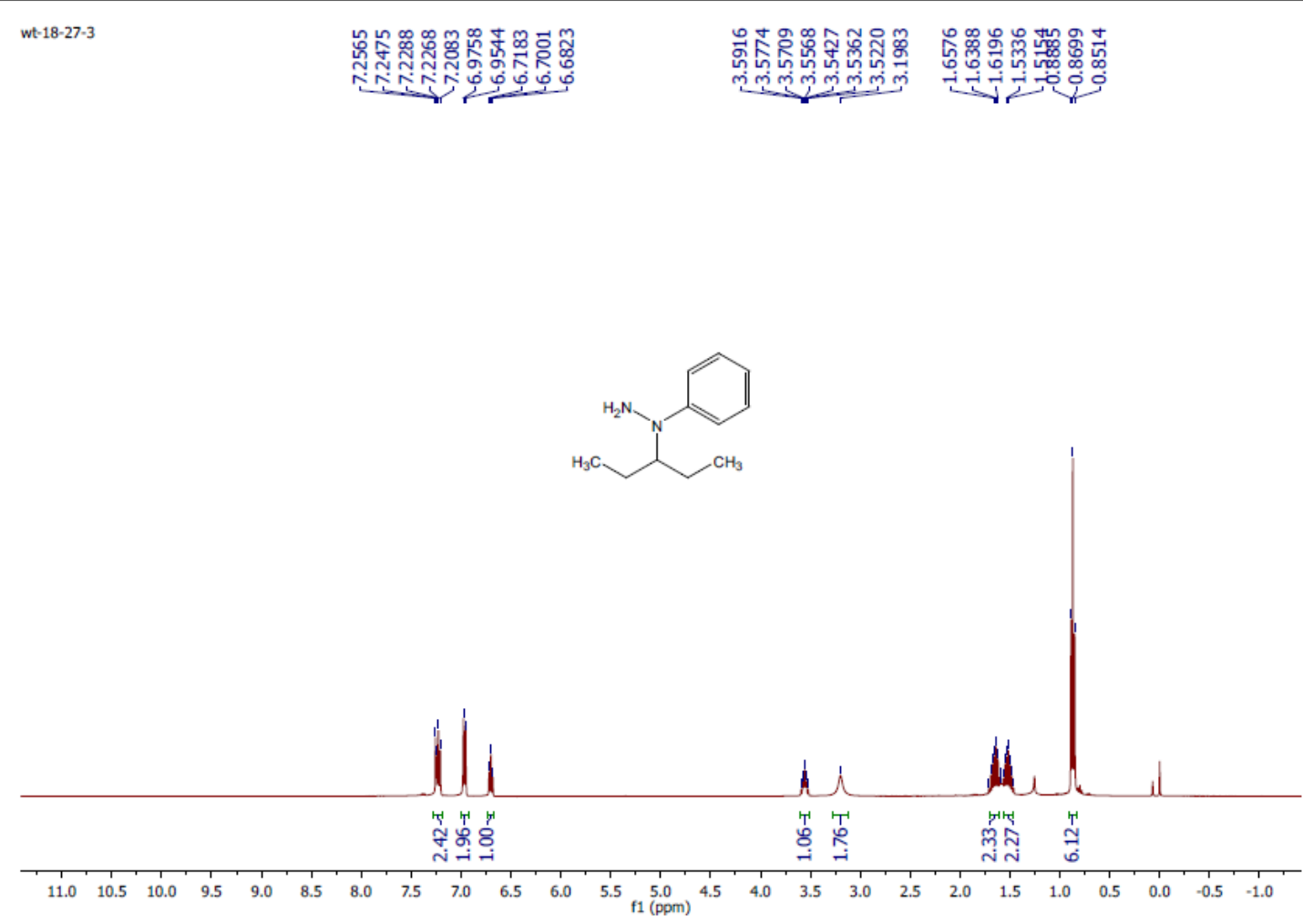

${ }^{13}$ C NMR of $3 x$

wt-18-27-3

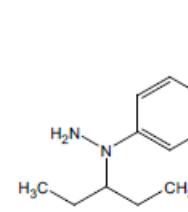


${ }^{1} \mathrm{H}$ NMR of $\mathbf{3 y}$

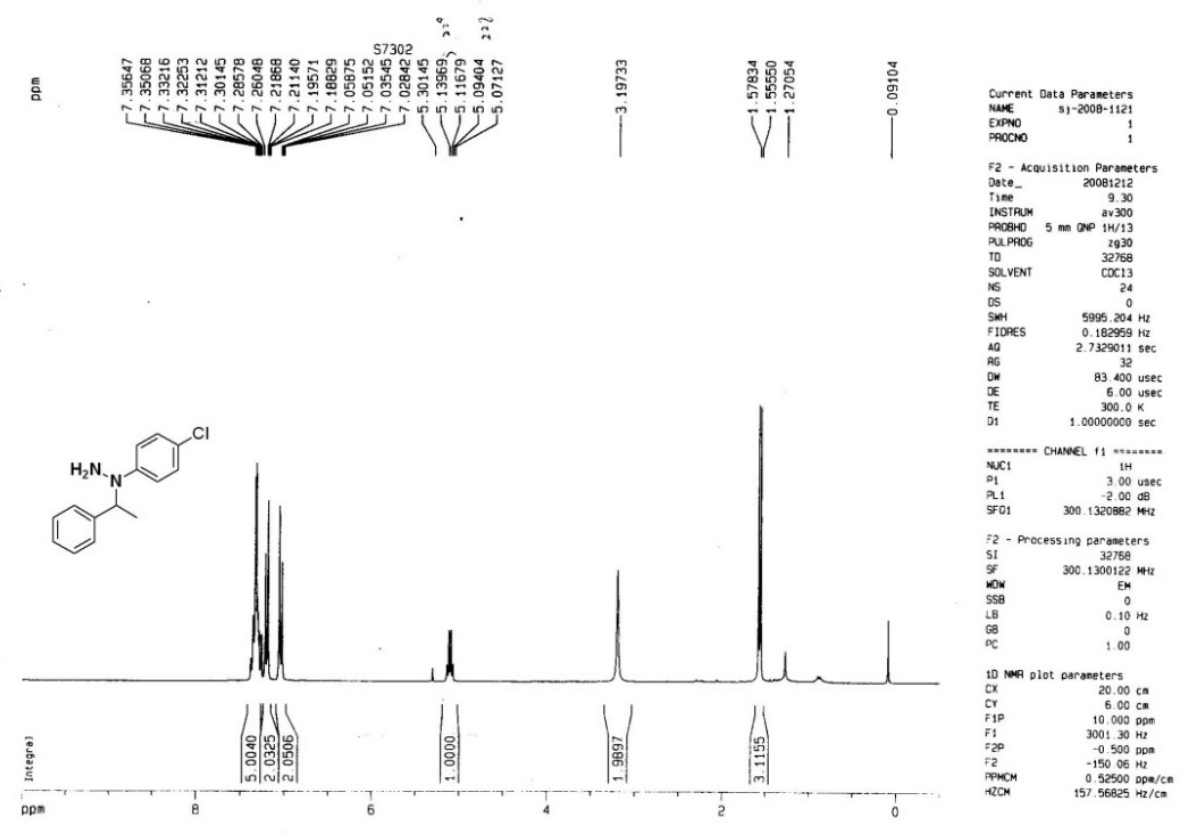

${ }^{13}$ C NMR of $3 y$

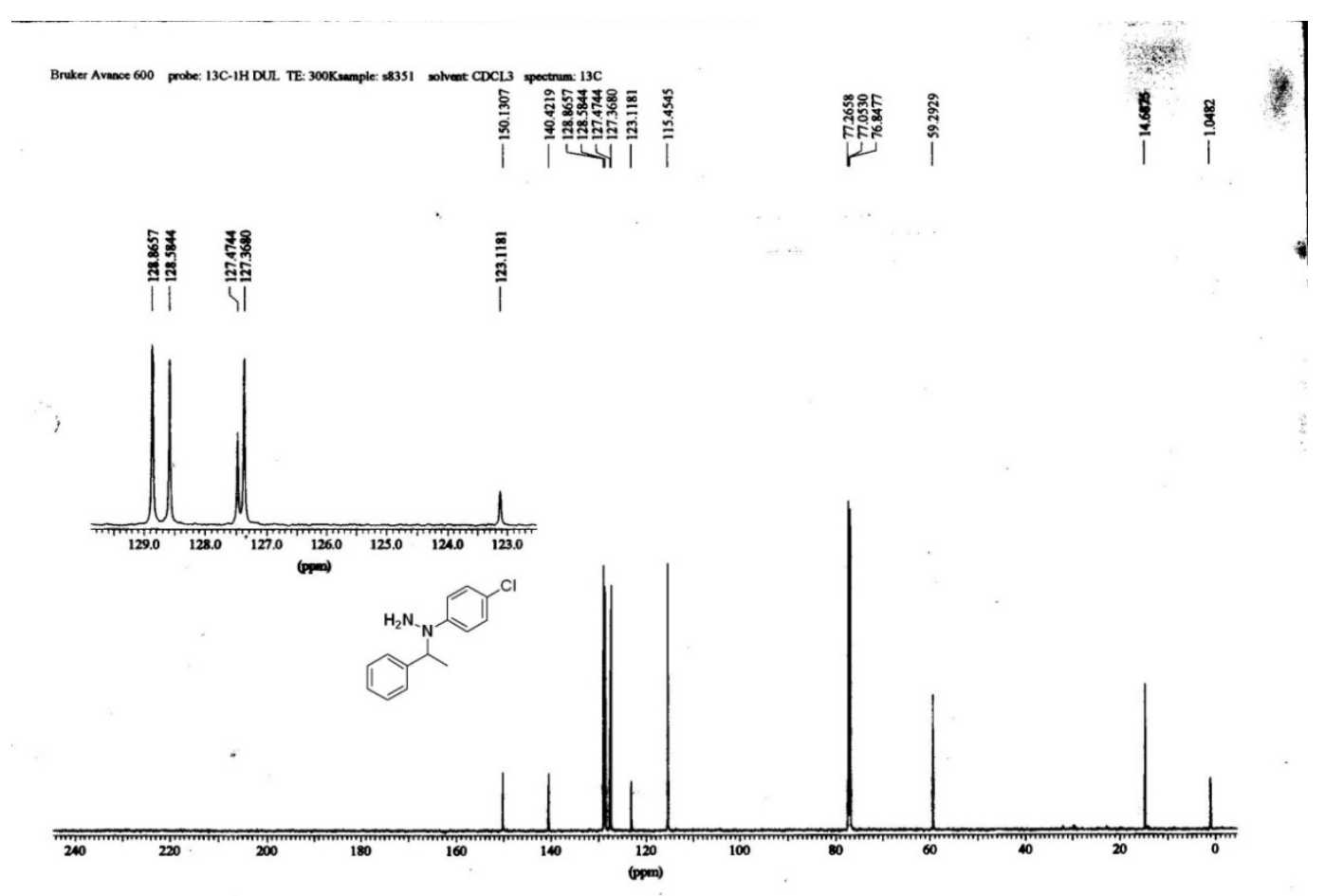


${ }^{1} \mathrm{H}$ NMR of $\mathbf{3 z}$

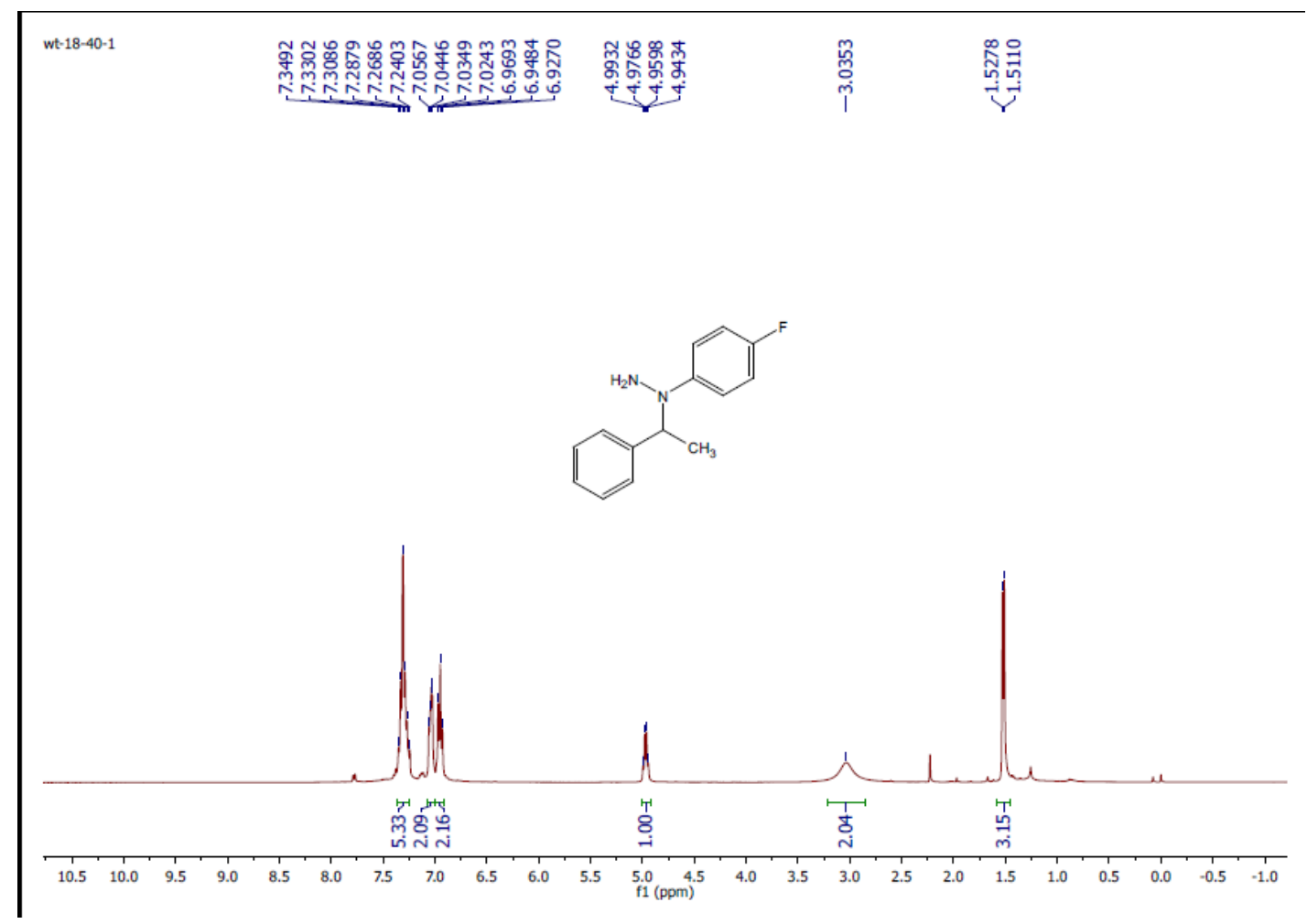

${ }^{13}$ C NMR of $3 z$

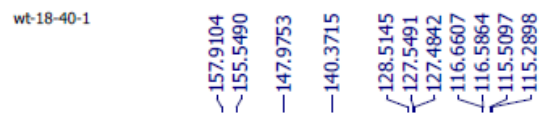
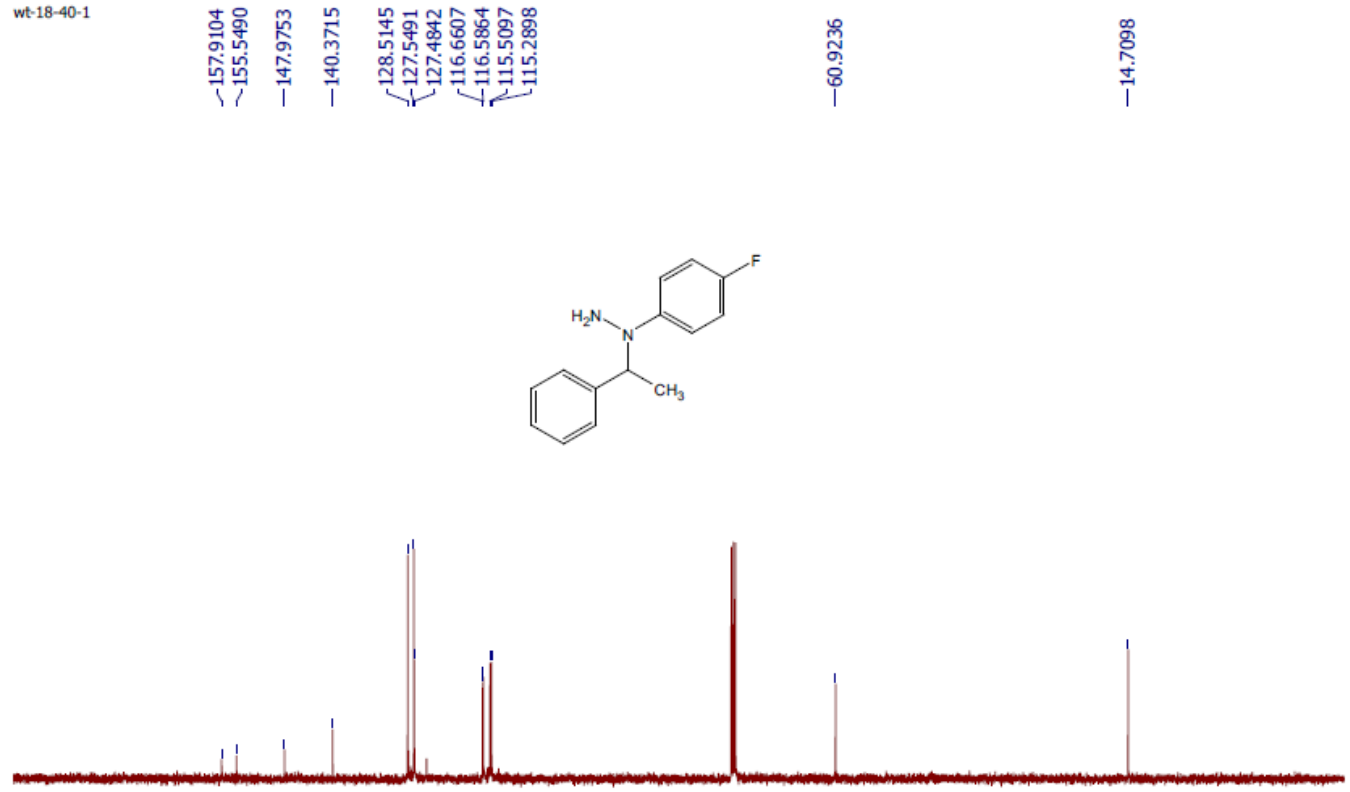

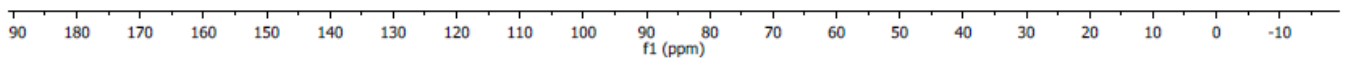


${ }^{1} \mathrm{H}$ NMR of $\mathbf{3 a a}$

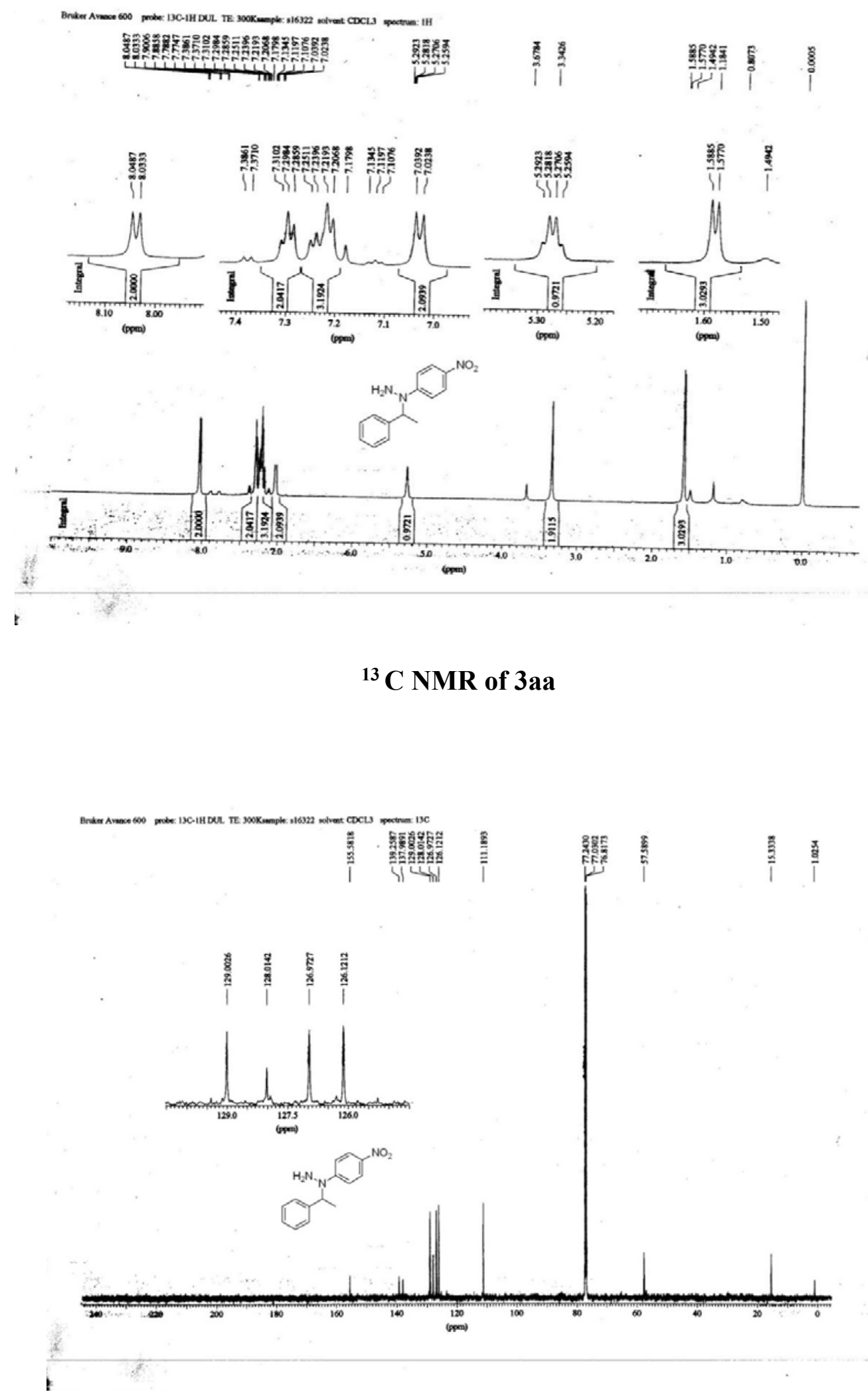


${ }^{1} \mathrm{H}$ NMR of $\mathbf{5 a}$

-
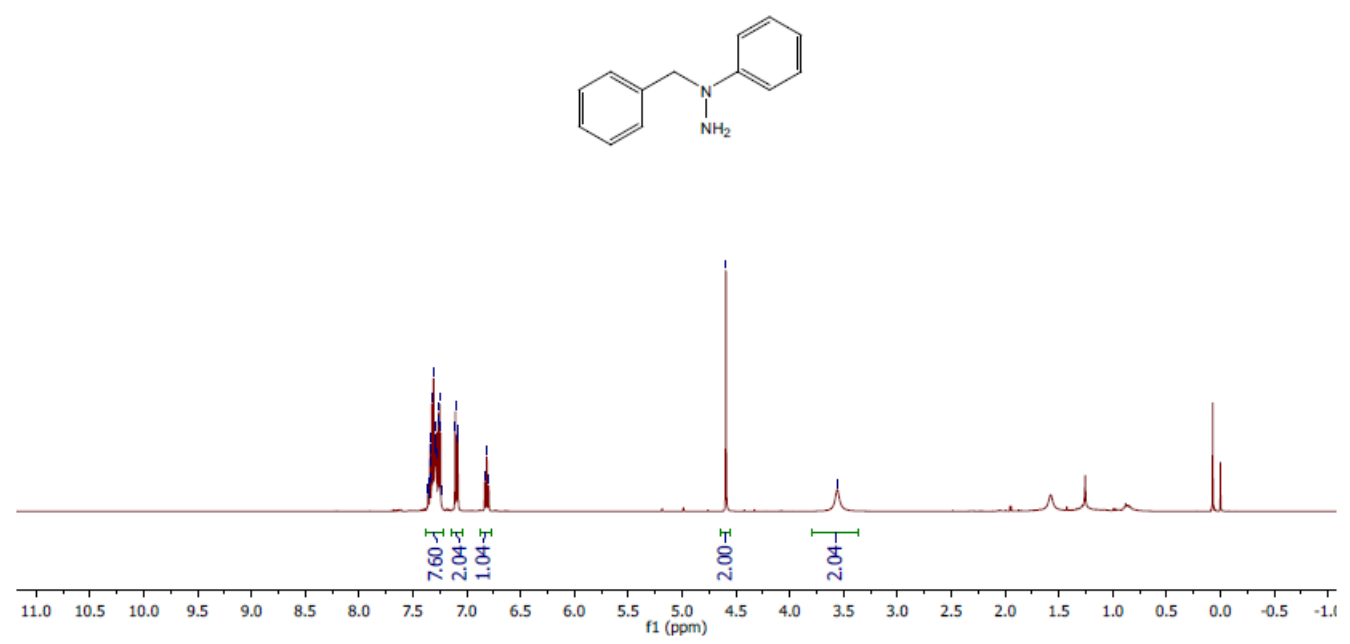

${ }^{13}$ C NMR of $5 a$

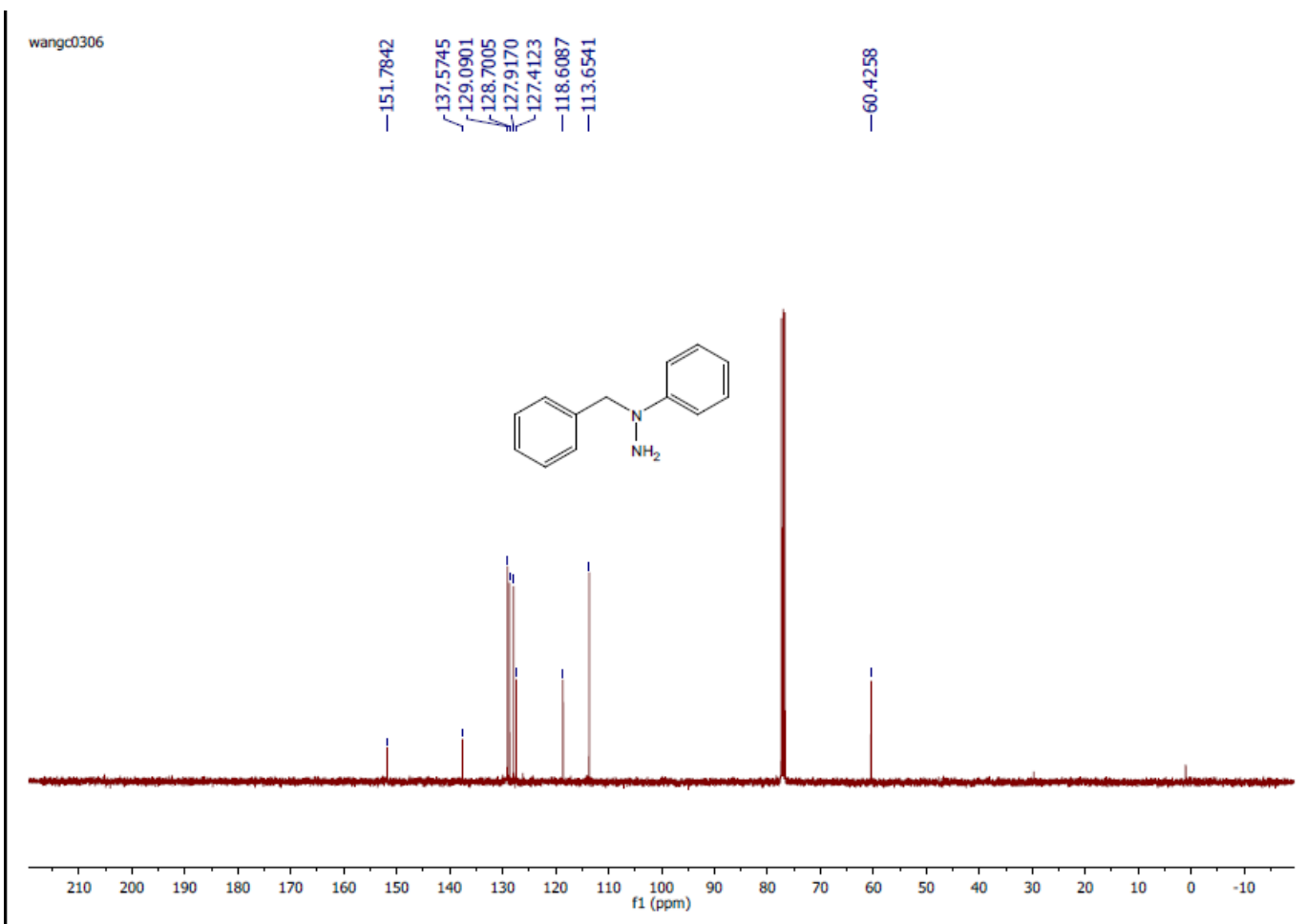


${ }^{1} \mathrm{H}$ NMR of $\mathbf{5 b}$

wt-18-32-3

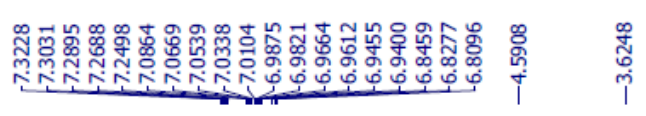

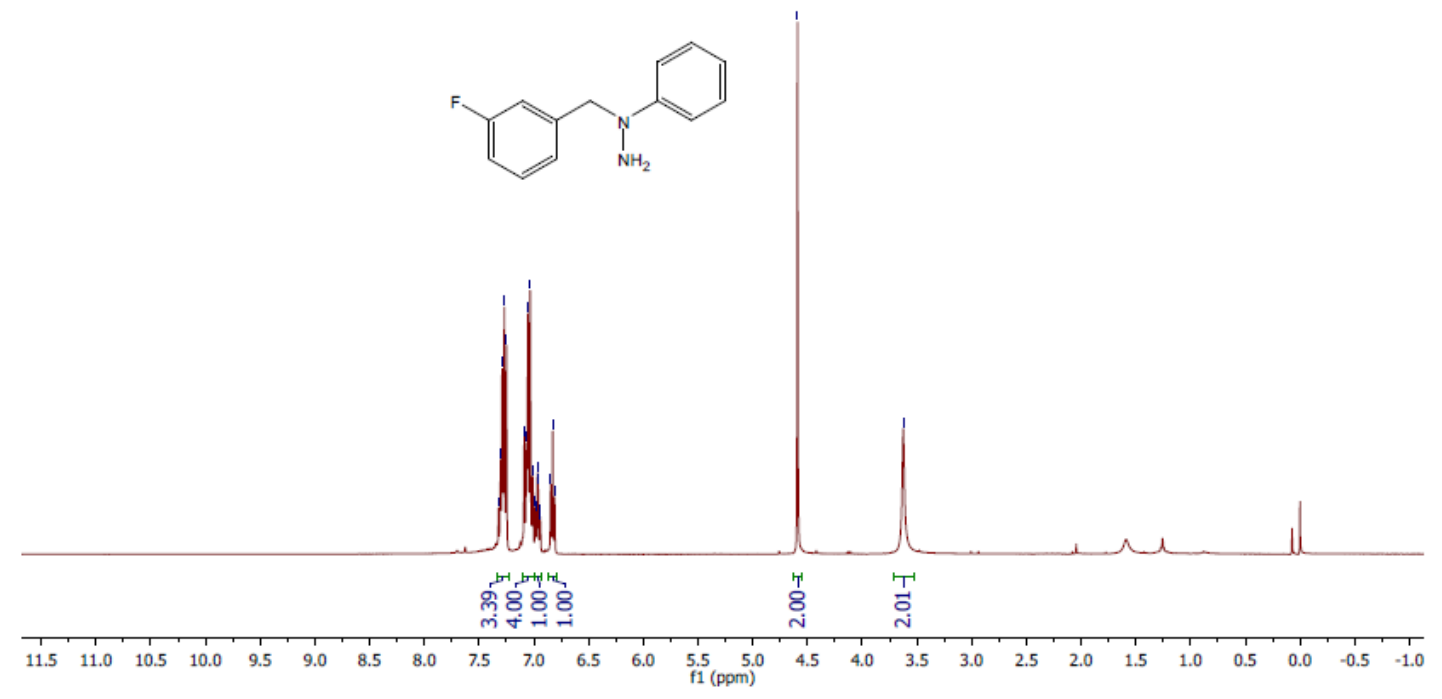

${ }^{13}$ C NMR of $5 b$

wt-18-32-3

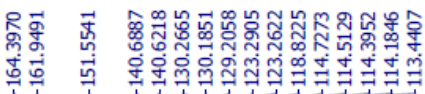

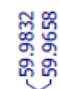

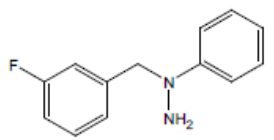

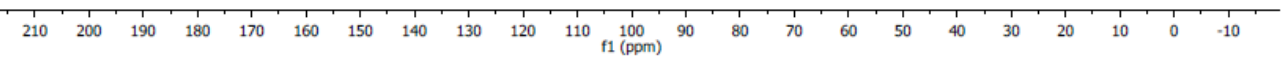


${ }^{1} \mathrm{H}$ NMR of $\mathbf{5 c}$

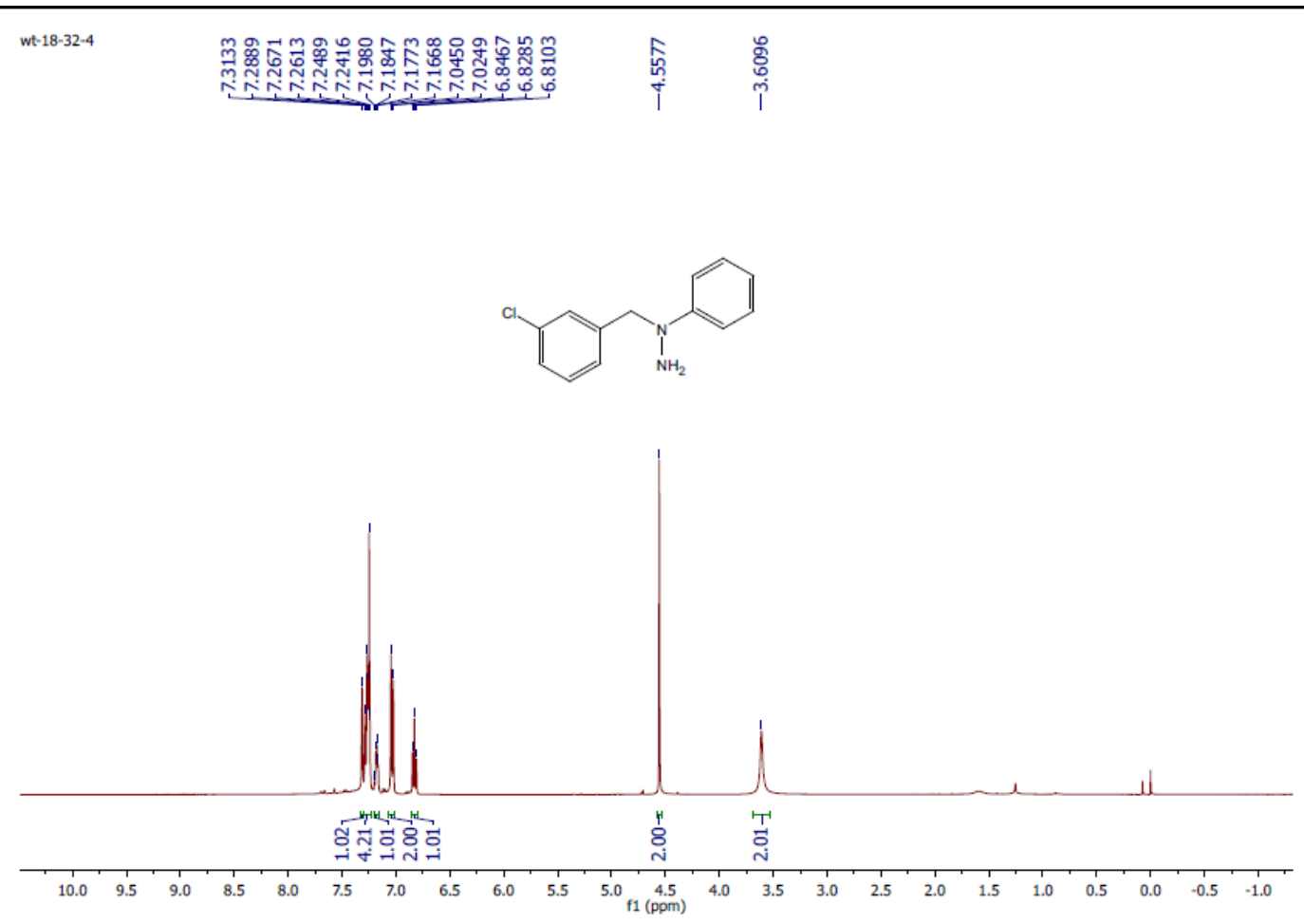

\section{${ }^{13}$ C NMR of $5 c$}

wt-18-32-4
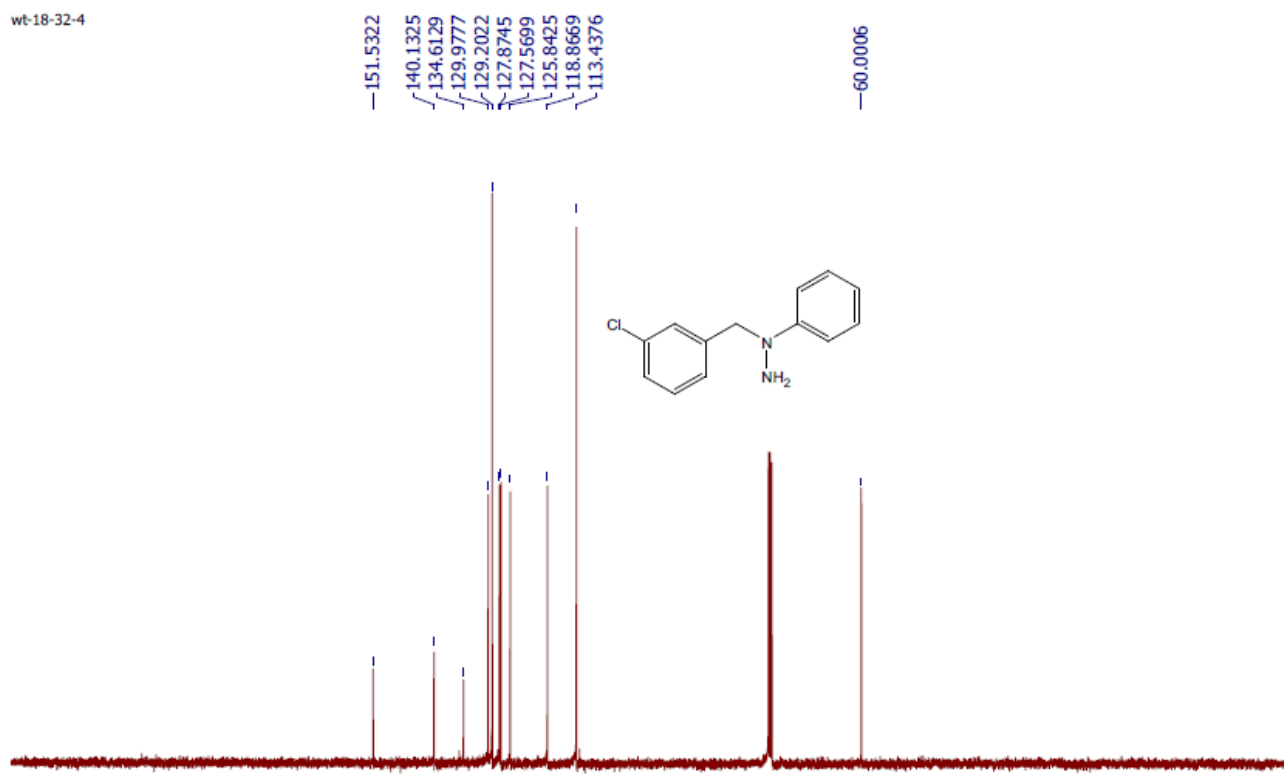

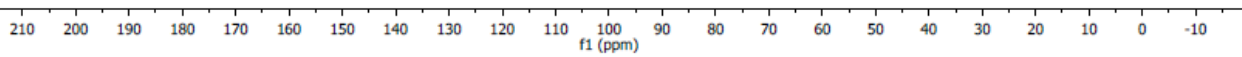


${ }^{1} \mathrm{H}$ NMR of $\mathbf{5 d}$

wt-18-30-2
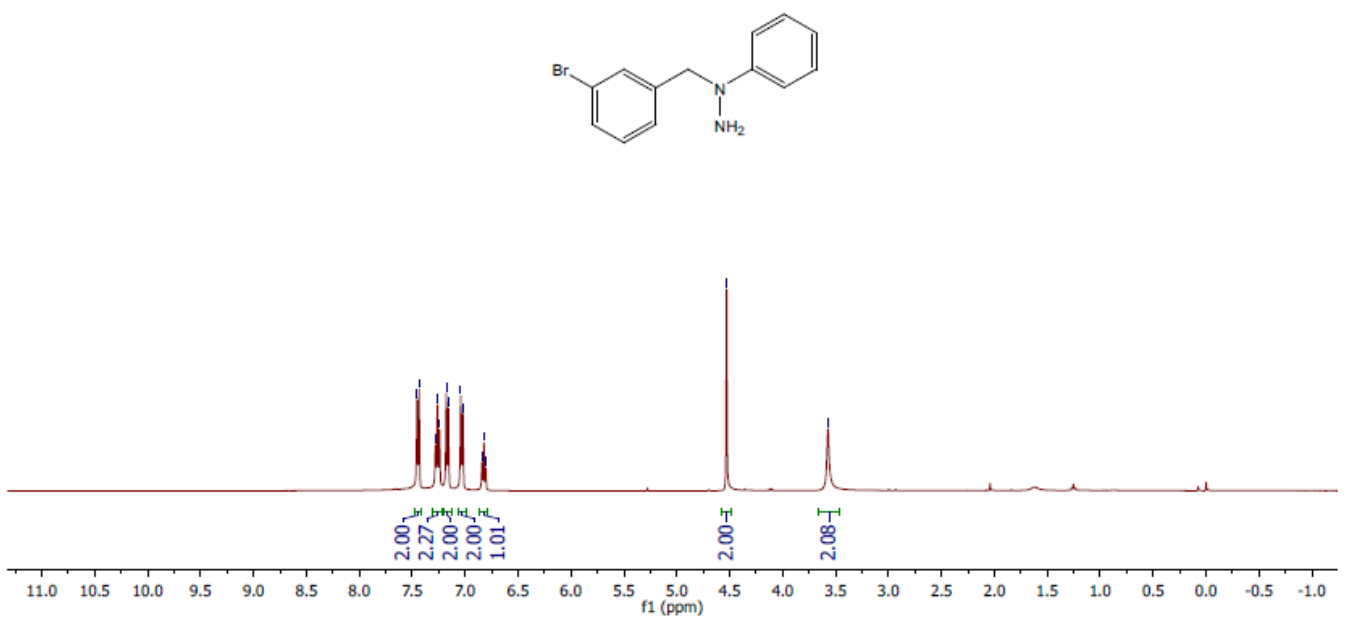

${ }^{13}$ C NMR of 5d

wang 0308

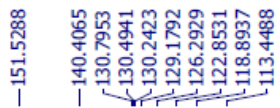

1
彳
0
0

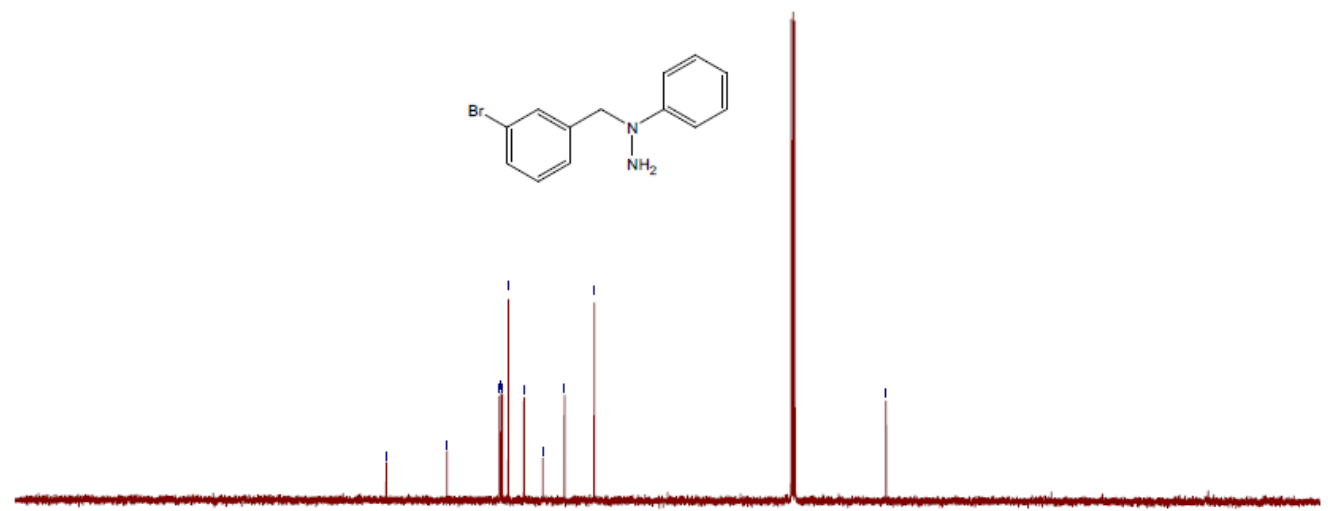

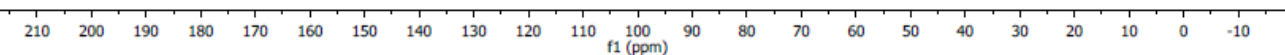


${ }^{1} \mathrm{H}$ NMR of $\mathbf{5 e}$

wo309

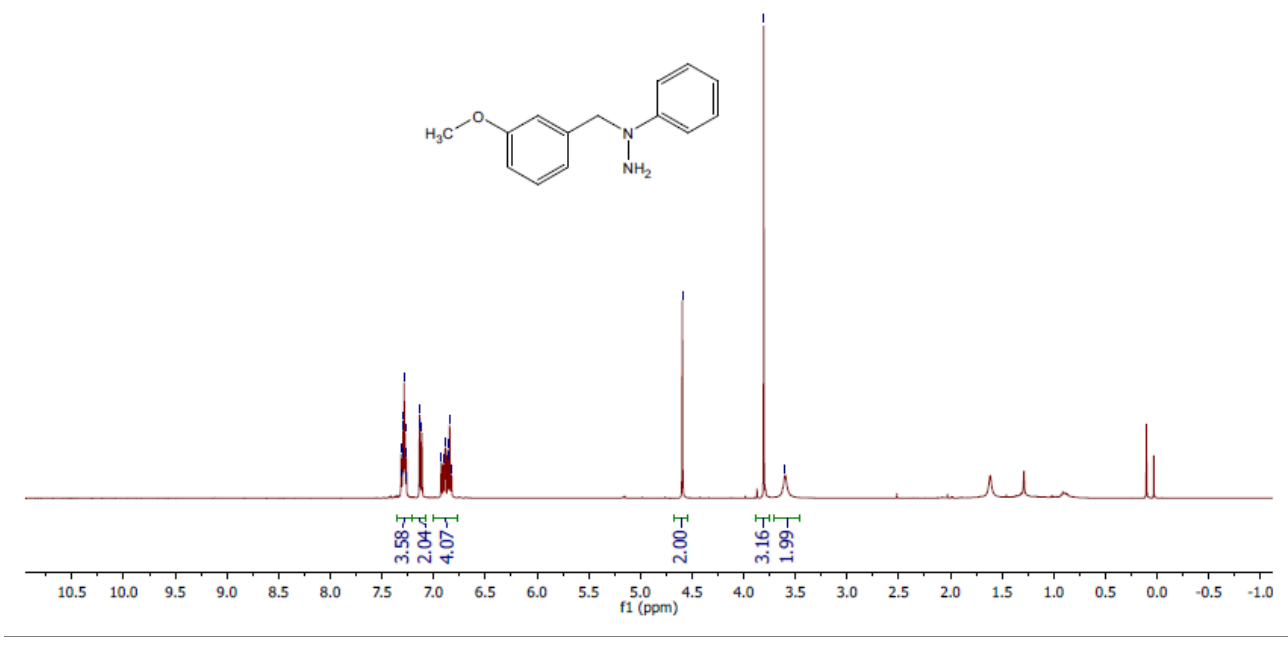

${ }^{13}$ C NMR of $5 e$

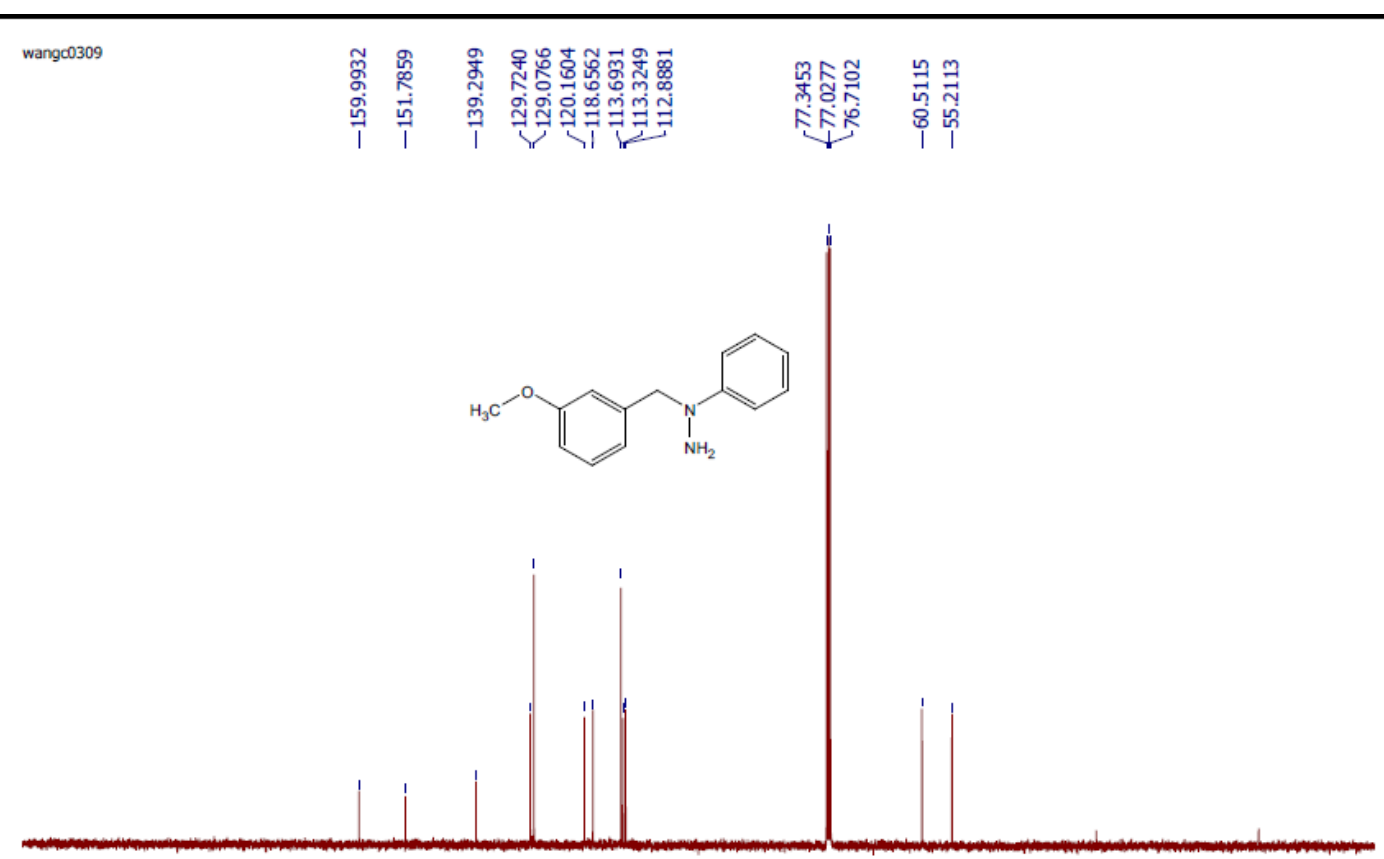

$\begin{array}{lllllllllllllllllllllllllllllllllllll}210 & 200 & 190 & 180 & 170 & 160 & 150 & 140 & 130 & 120 & 110 & 100 & 90 & 80 & 70 & 60 & 50 & 40 & 30 & 20 & 10 & 0 & -10\end{array}$ 
${ }^{1} \mathrm{H}$ NMR of $\mathbf{5 f}$

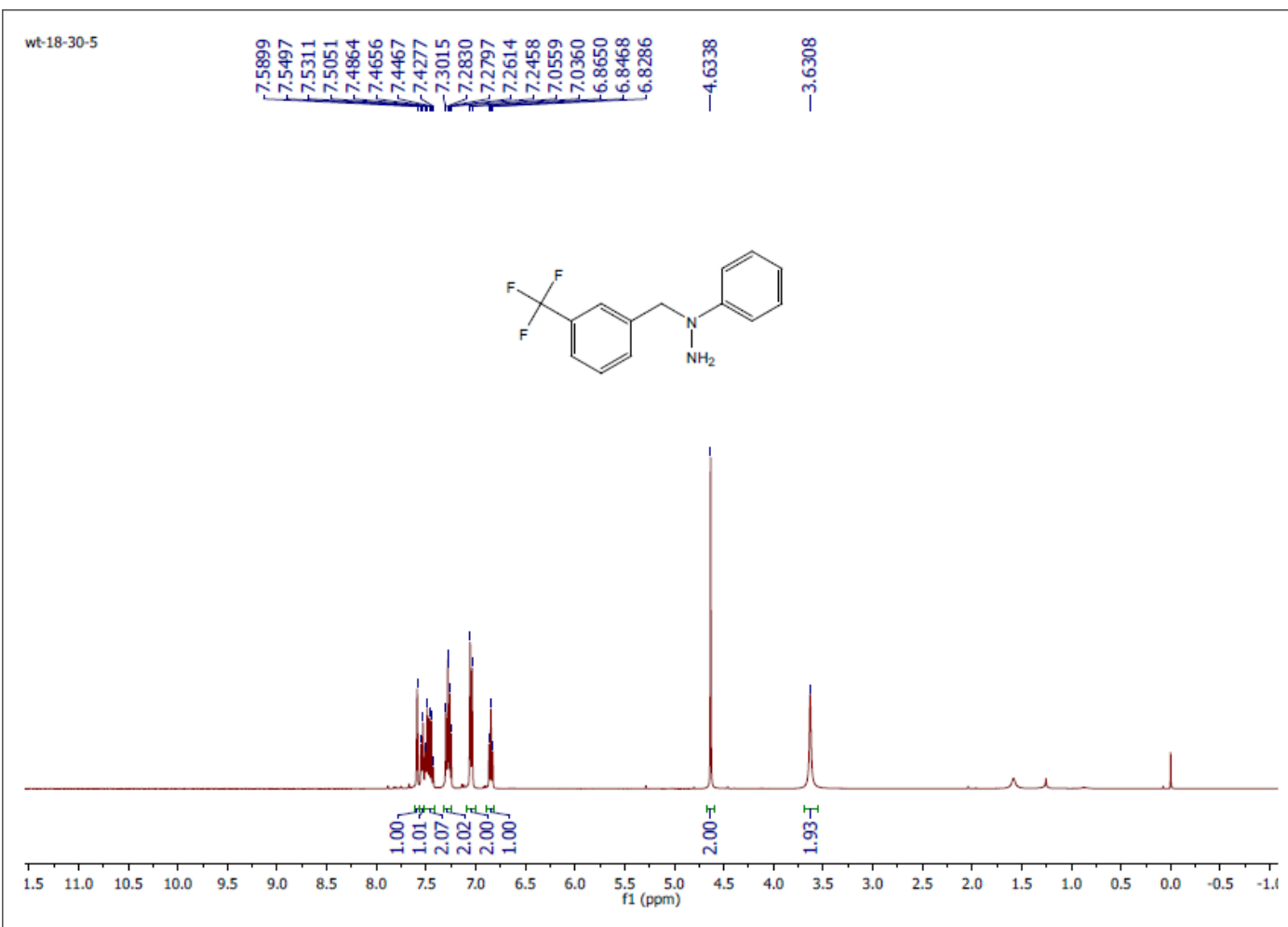

${ }^{13} \mathrm{C}$ NMR of $5 f$

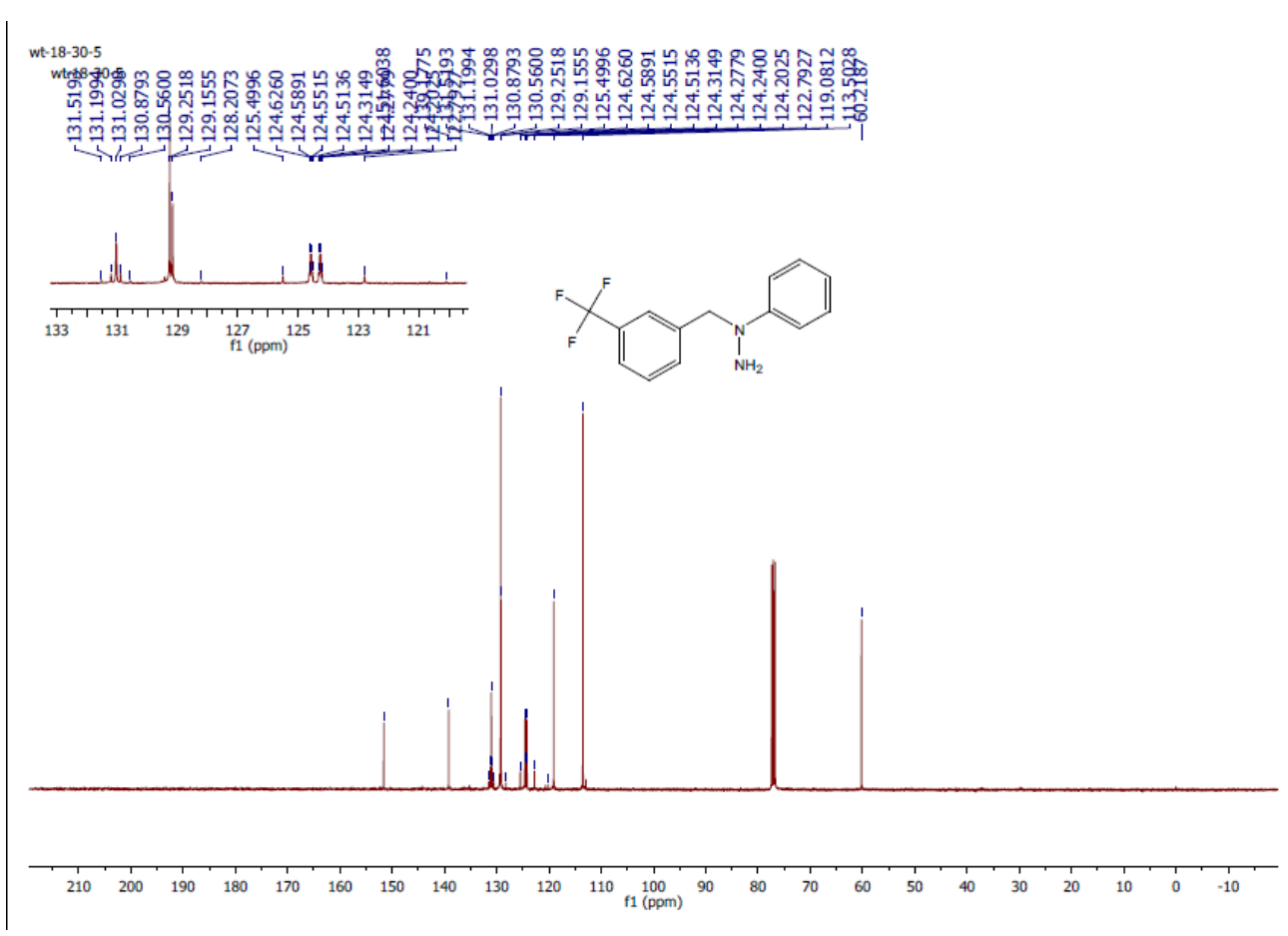


${ }^{1} \mathrm{H}$ NMR of $\mathbf{5 g}$

wt-18-30-6

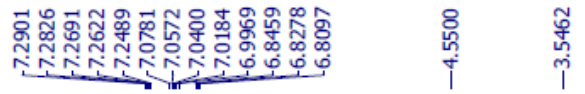

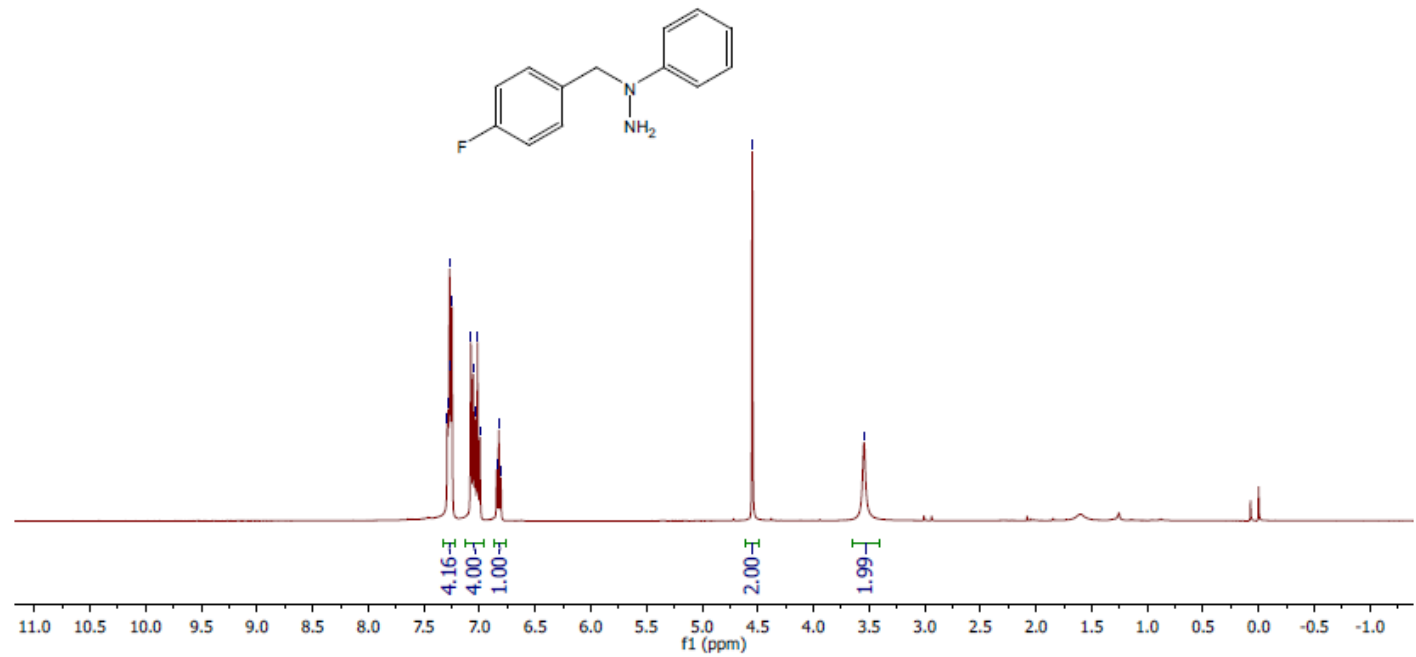

${ }^{13}$ C NMR of $5 g$

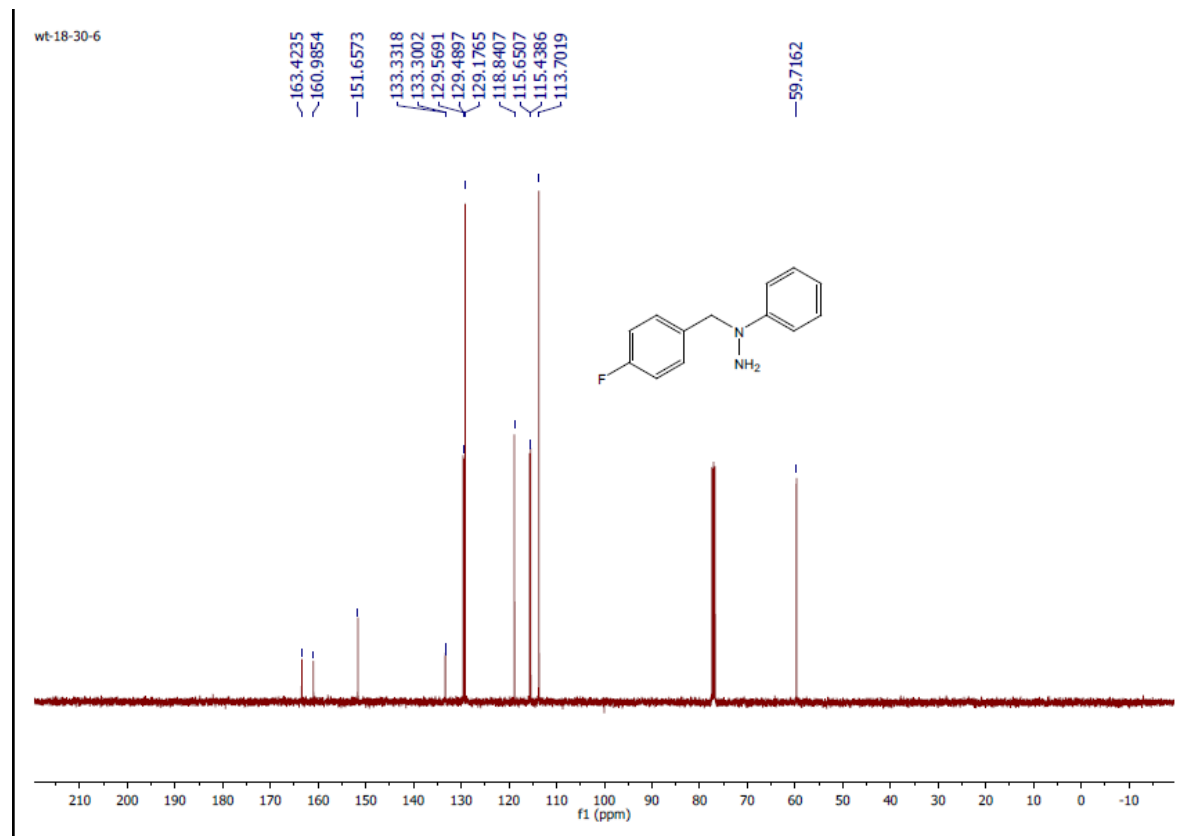


${ }^{1} \mathrm{H}$ NMR of $\mathbf{5 h}$

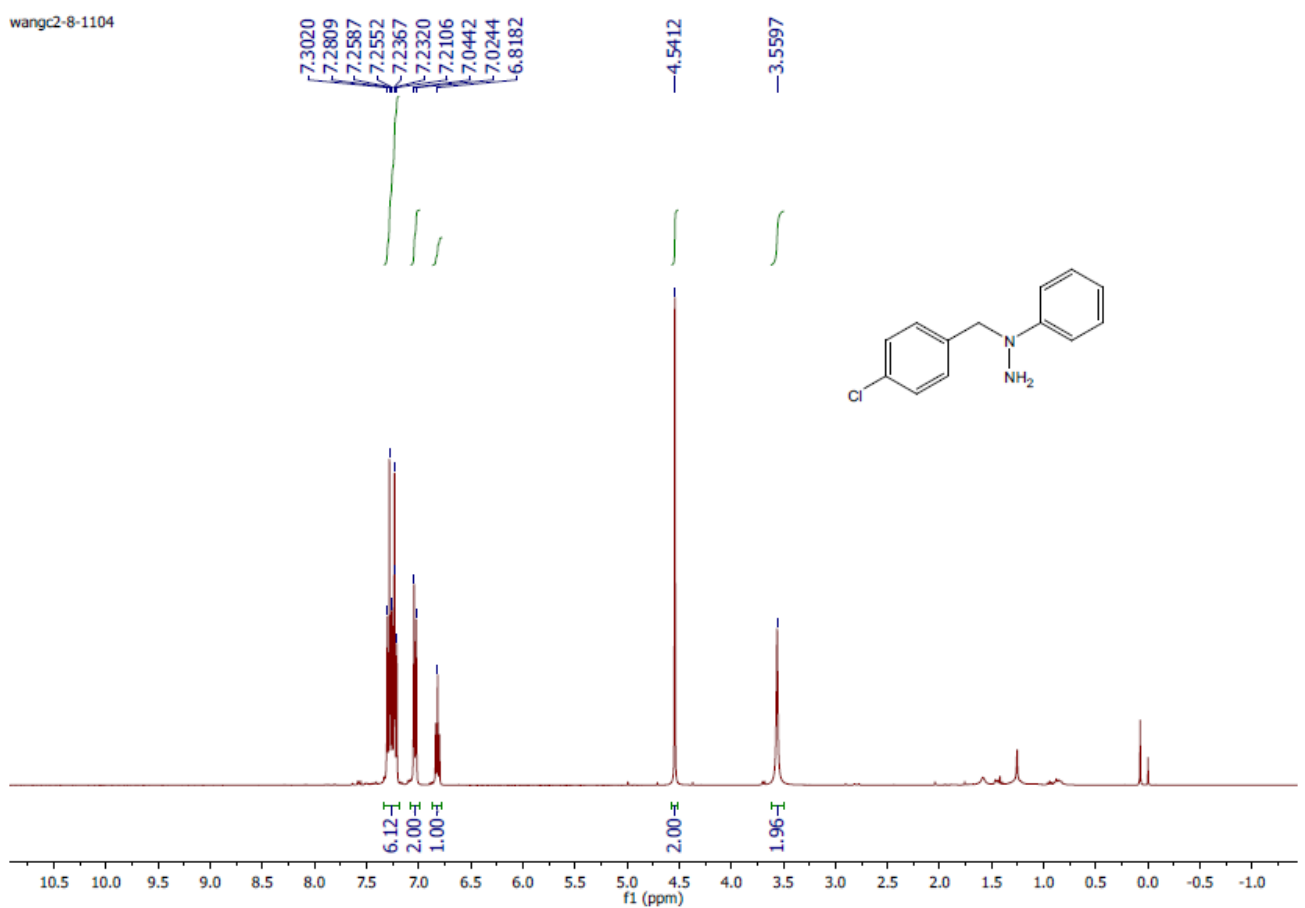

${ }^{13} \mathrm{C}$ NMR of $5 \mathrm{~h}$

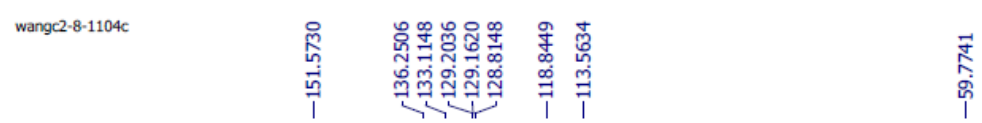

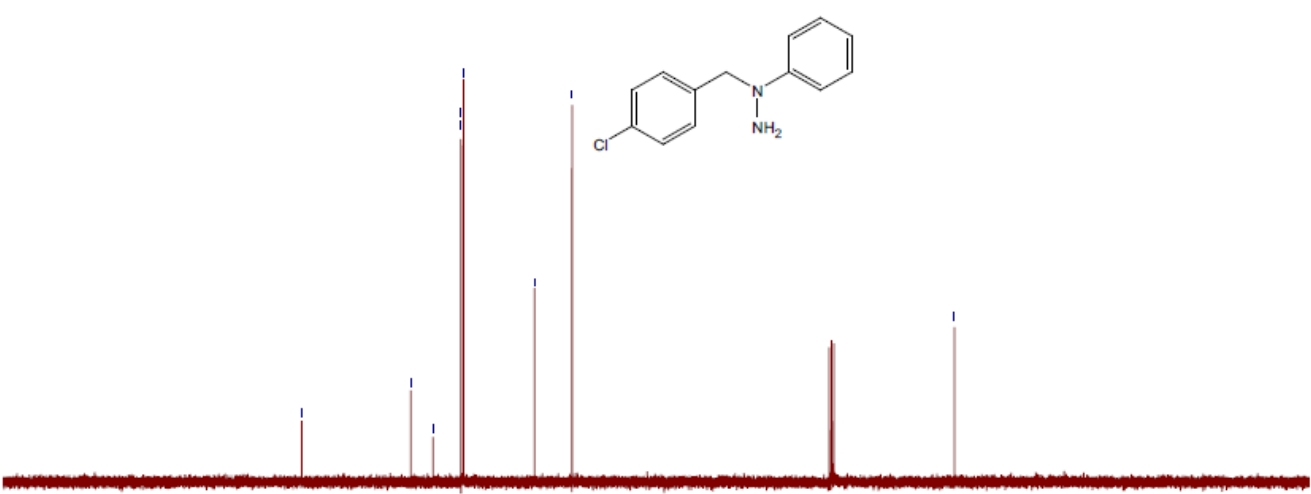

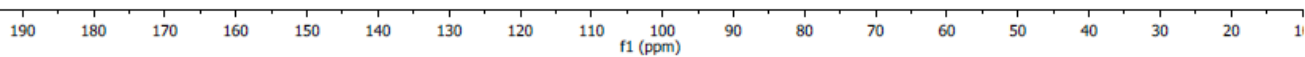


${ }^{1} \mathrm{H}$ NMR of $\mathbf{5 i}$

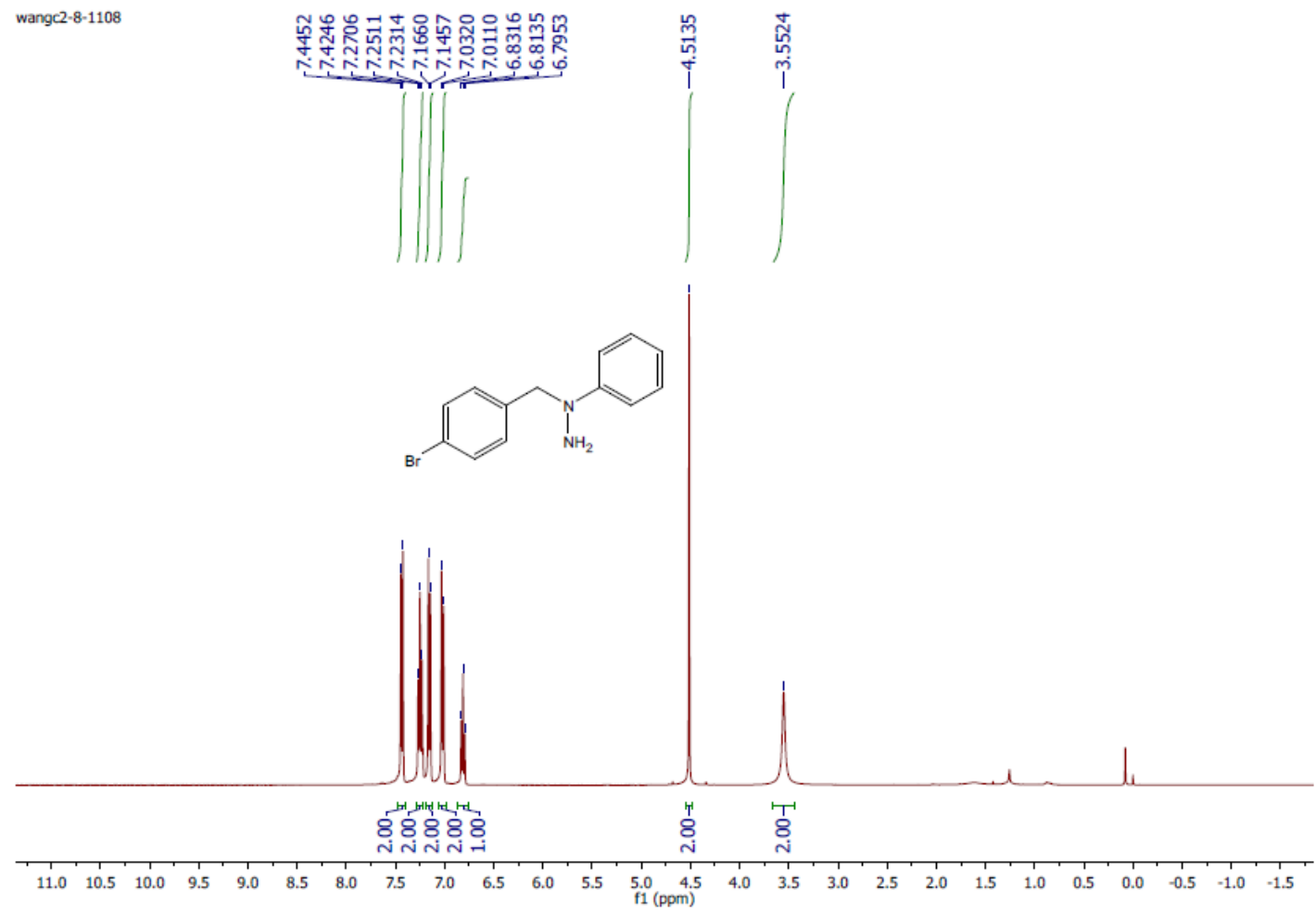

${ }^{13}$ C NMR of $5 i$

wangc2-8-1108c

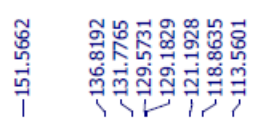

员

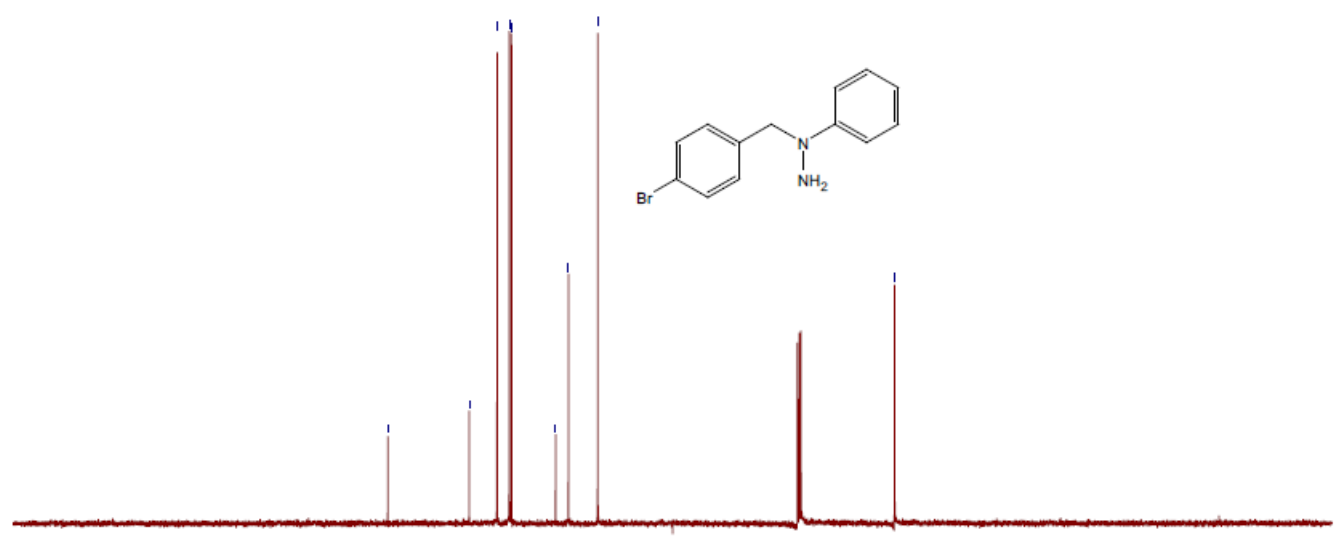

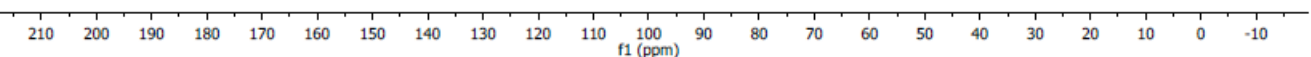


${ }^{1} \mathrm{H}$ NMR of $\mathbf{5 j}$

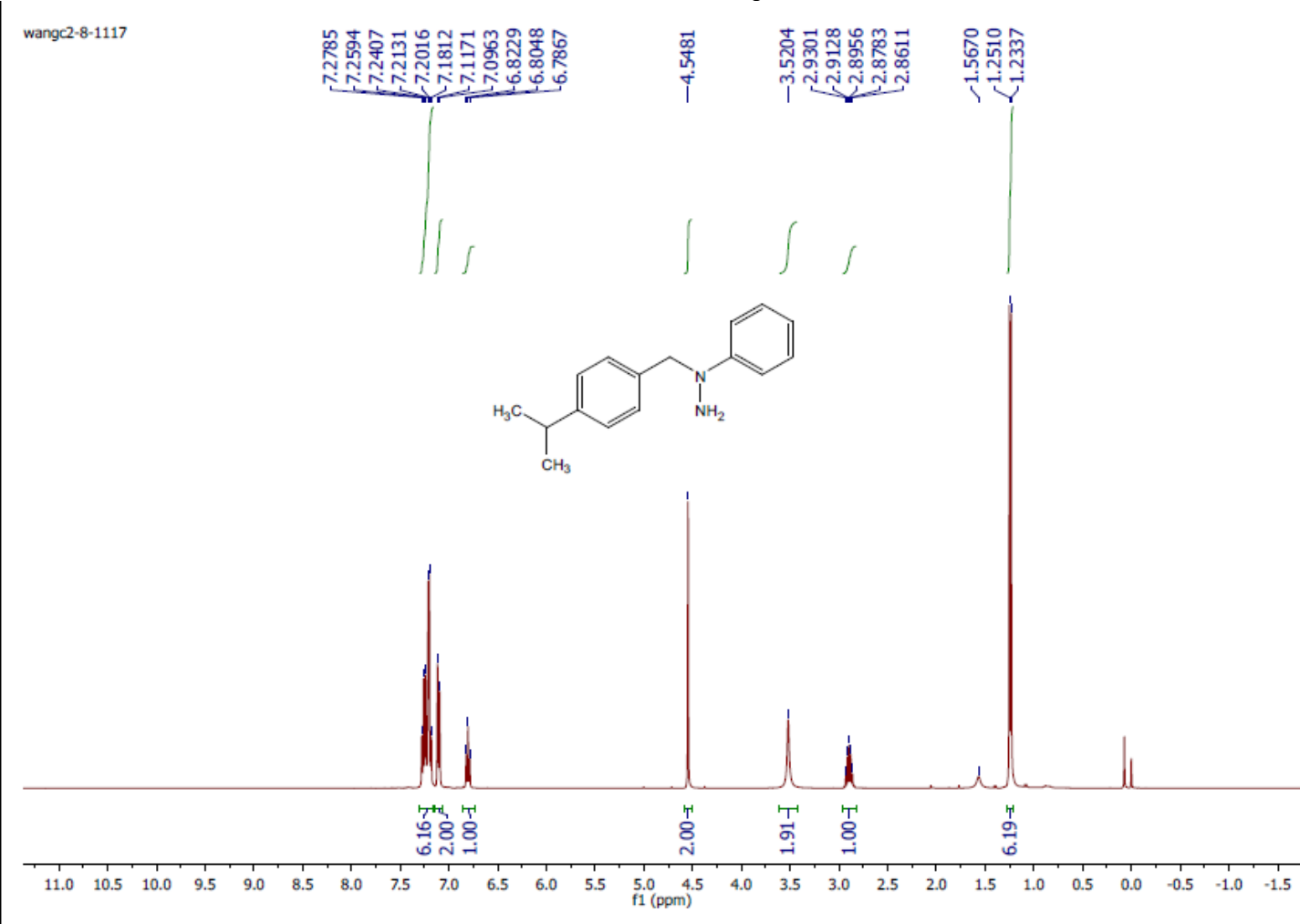

${ }^{13}$ C NMR of $5 j$

wangc2-8-1117c
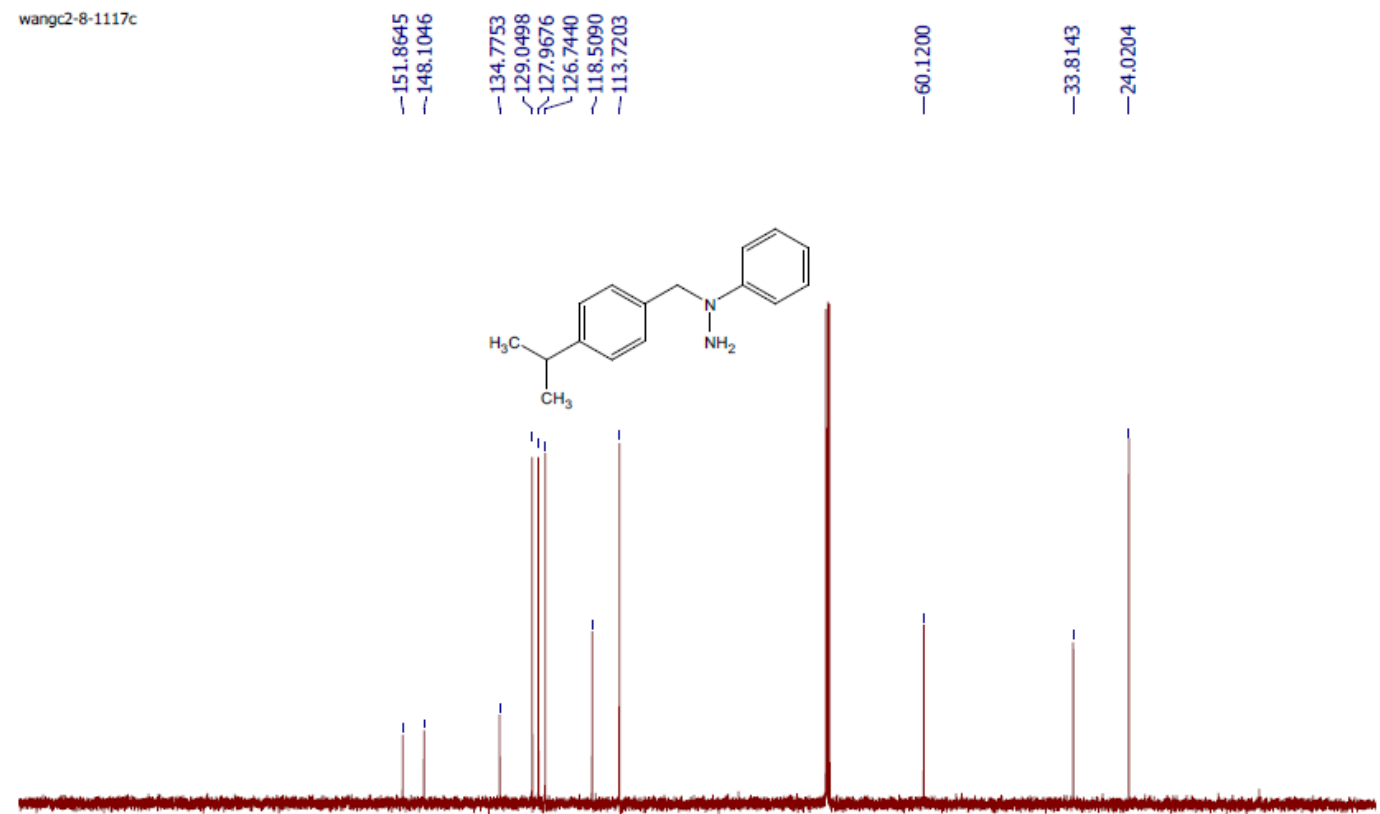

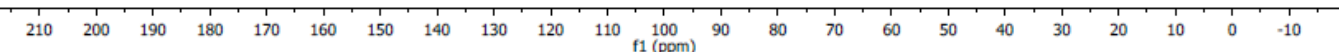


${ }^{1} \mathrm{H}$ NMR of $\mathbf{5 k}$

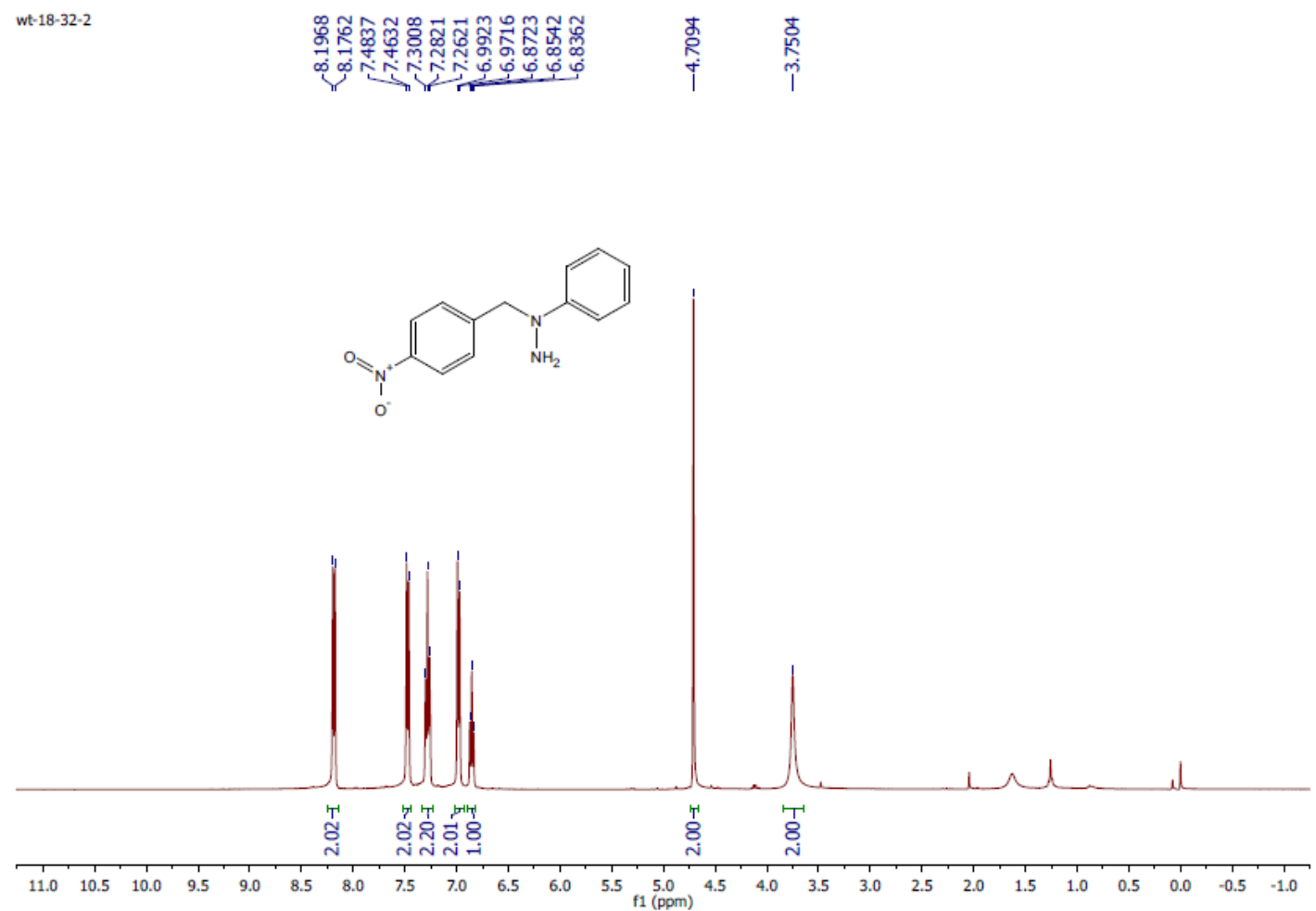

${ }^{13}$ C NMR of $5 k$

wt-18-32-2

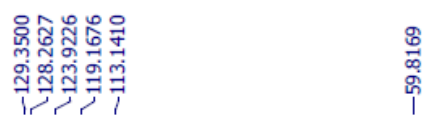

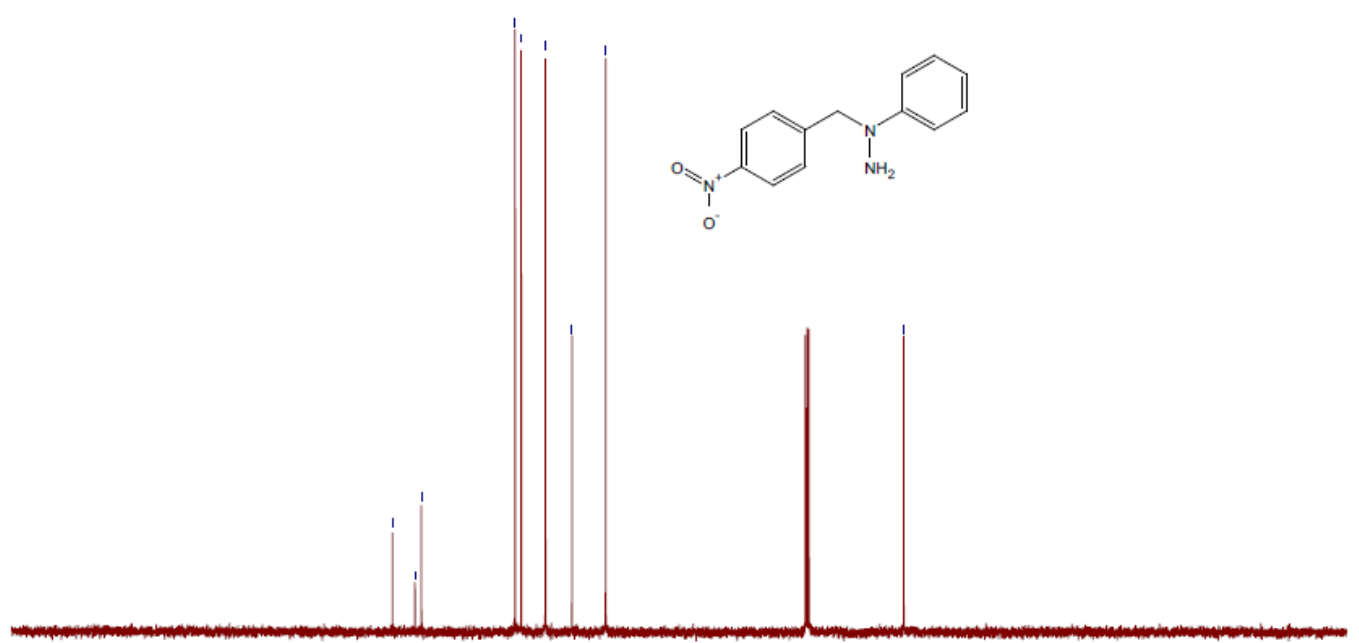

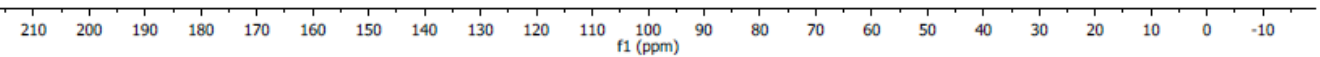




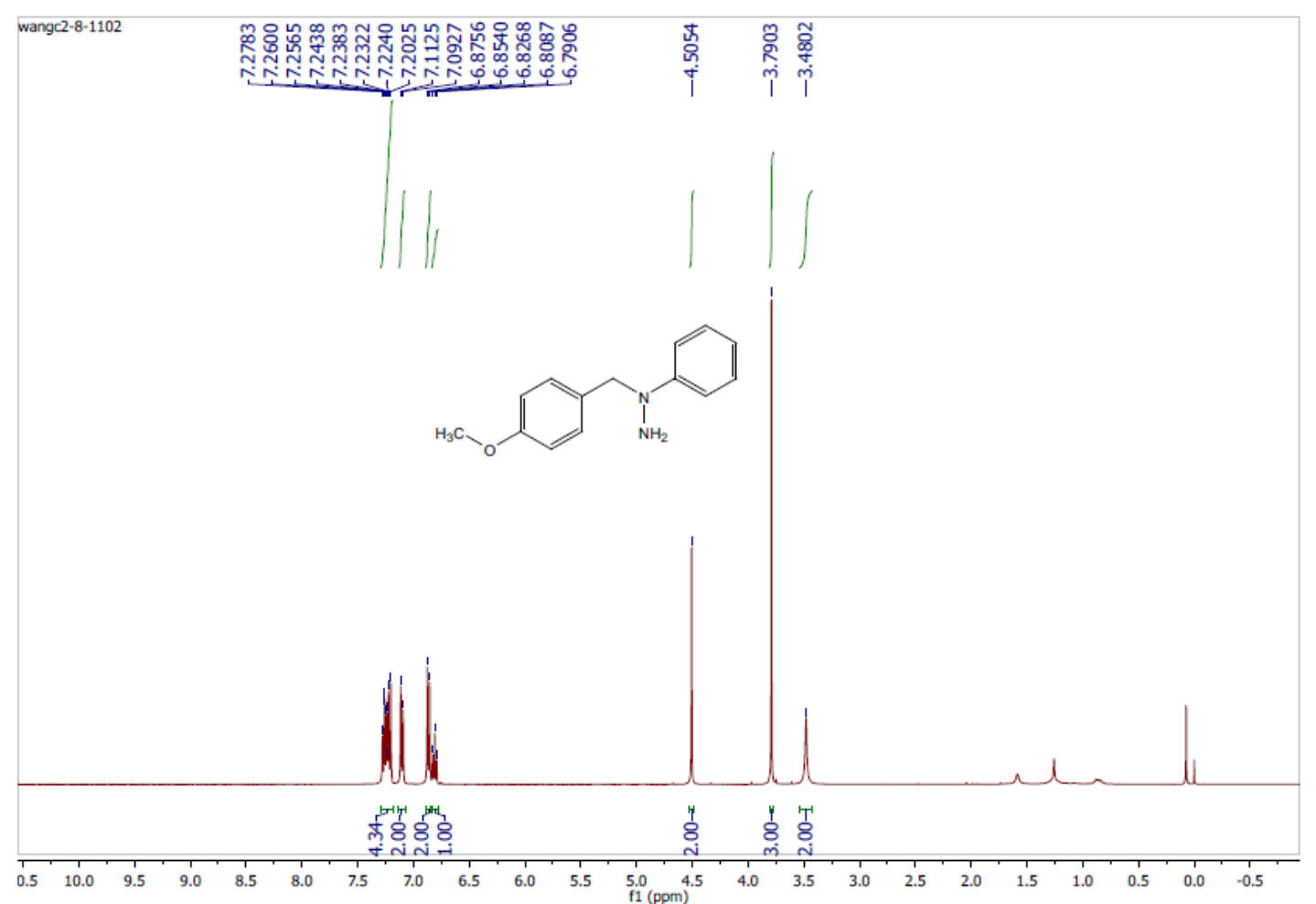

${ }^{13}$ C NMR of 5 I

wangc2-8-1102c

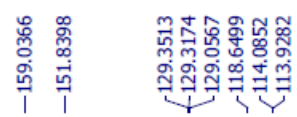

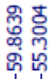
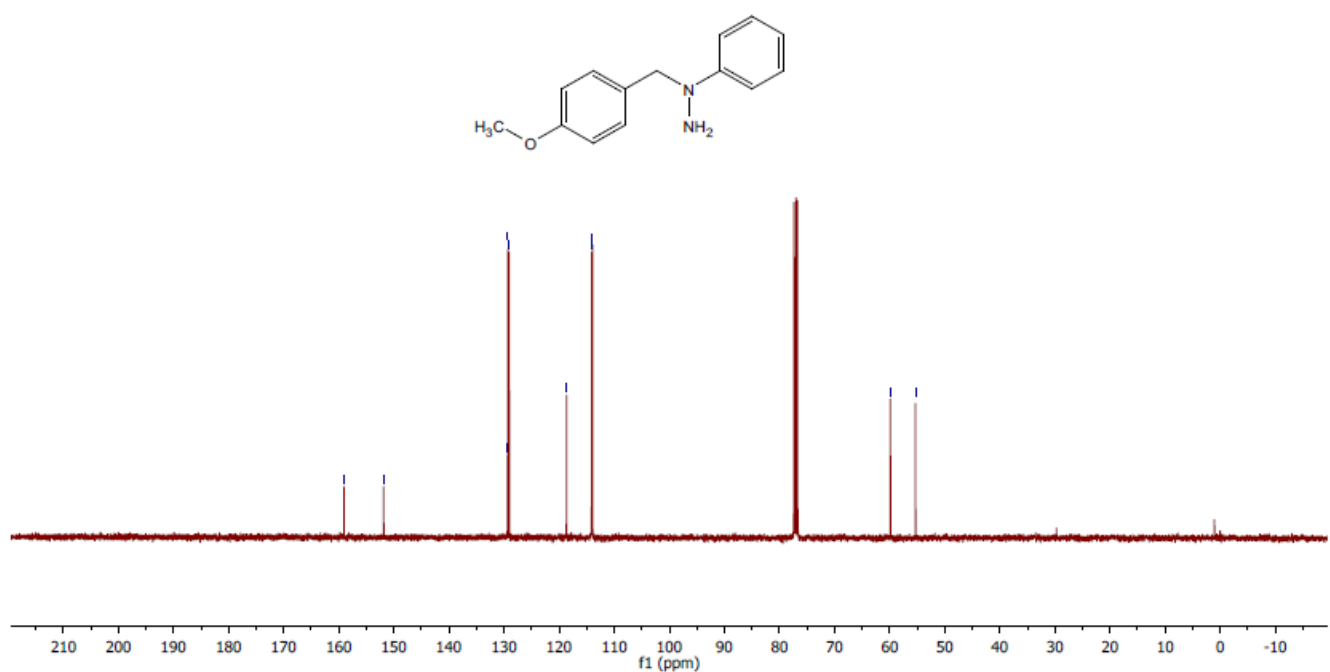
${ }^{1} \mathrm{H}$ NMR of $\mathbf{5 m}$

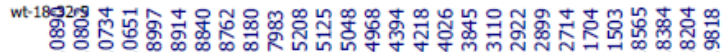

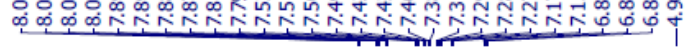

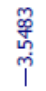

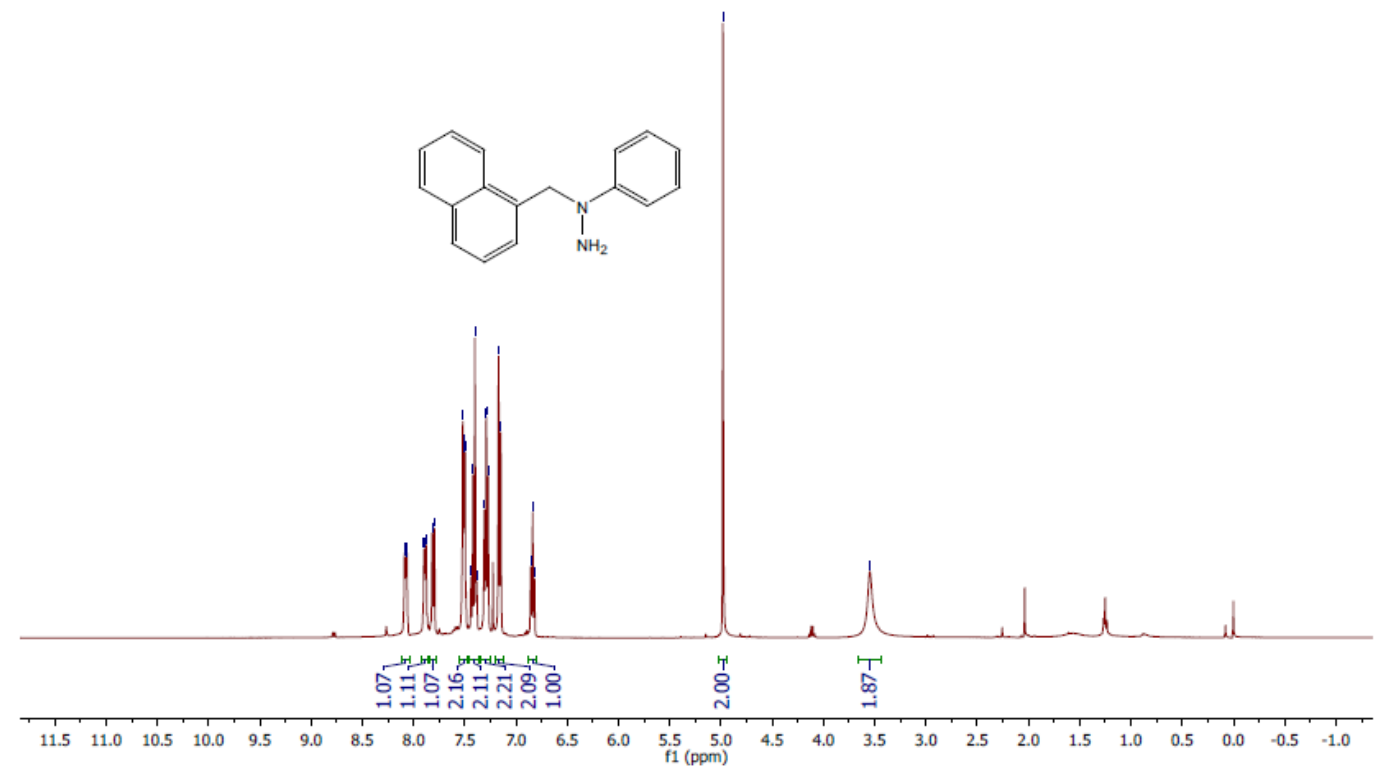

${ }^{13}$ C NMR of $5 \mathrm{~m}$

\begin{tabular}{|c|c|}
\hline wt-18-32-5 & 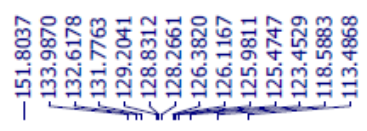 \\
\hline
\end{tabular}

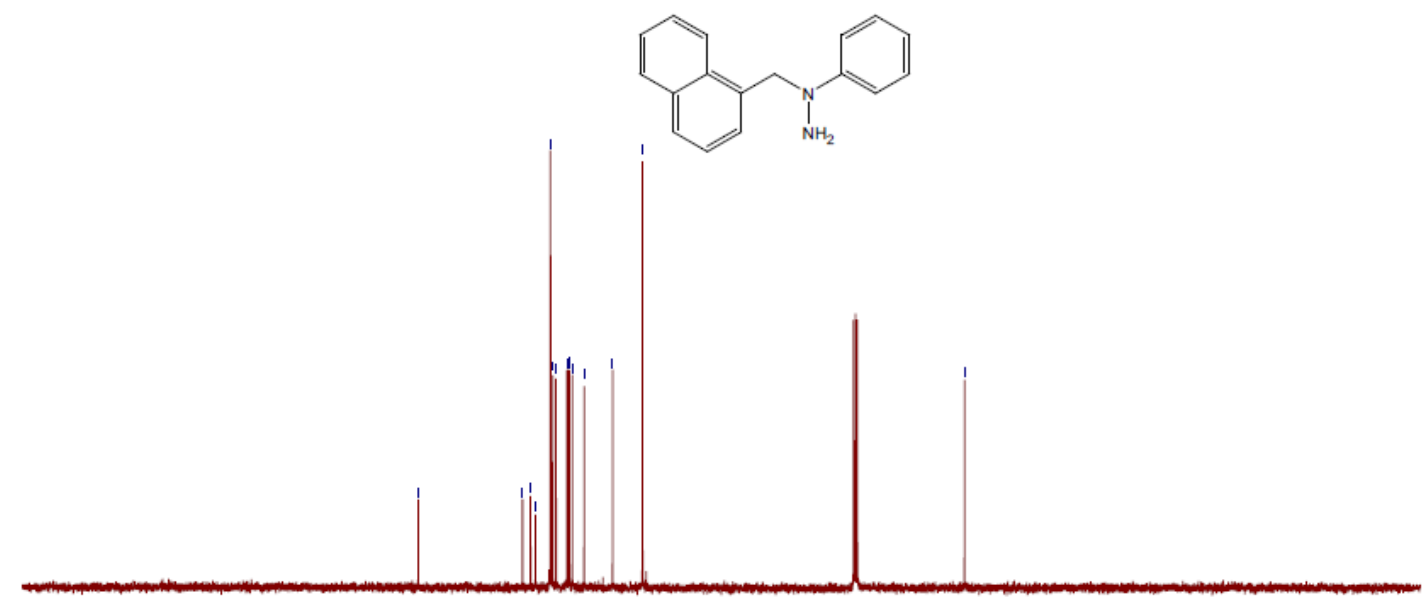

$\begin{array}{lllllllllllllllllllllllllllllll}210 & 200 & 190 & 180 & 170 & 160 & 150 & 140 & 130 & 120 & 110 & 100 & 9 & 80 & 70 & 60 & 50 & 40 & 30 & 20 & 10 & 0 & -10\end{array}$ 
${ }^{1} \mathrm{H}$ NMR of $\mathbf{5 n}$

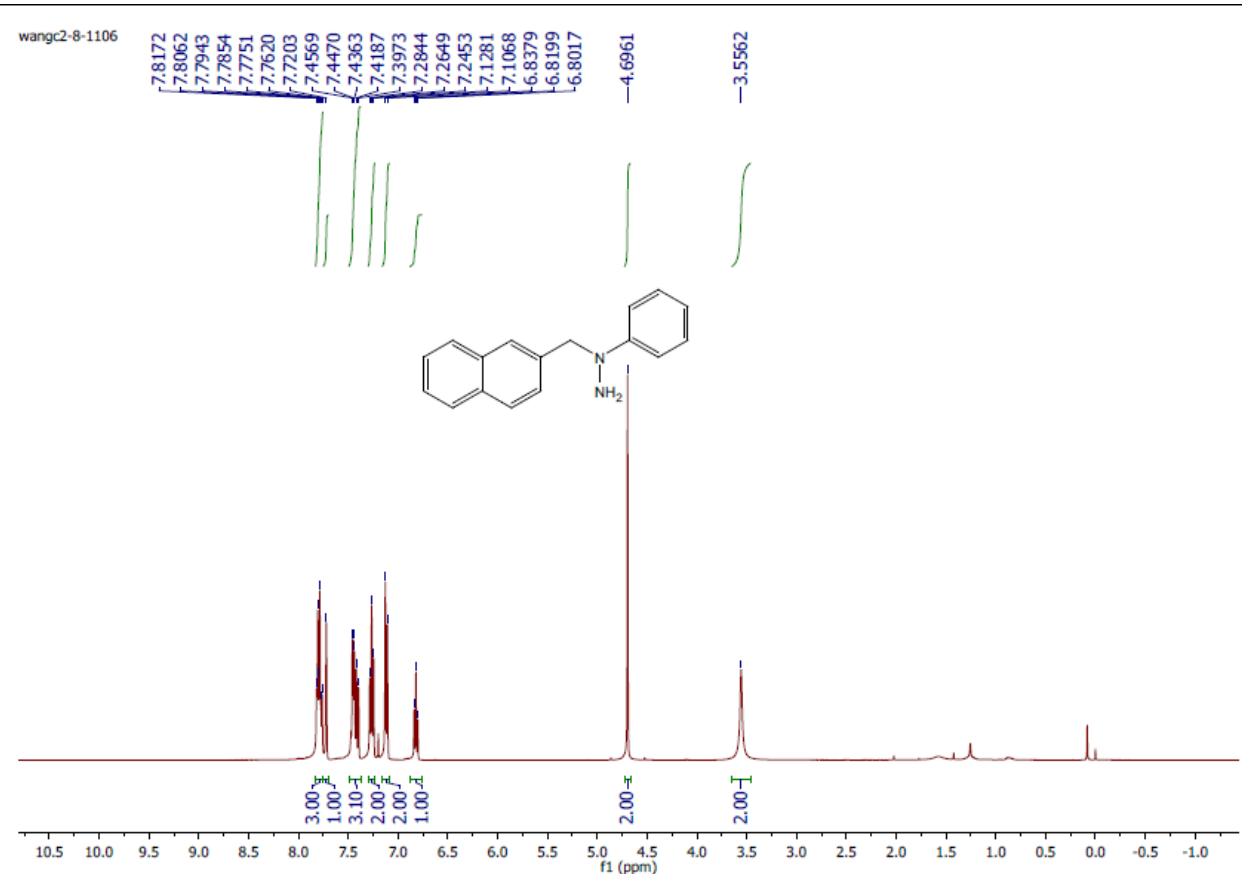

${ }^{13}$ C NMR of

wangc2-8-1106c
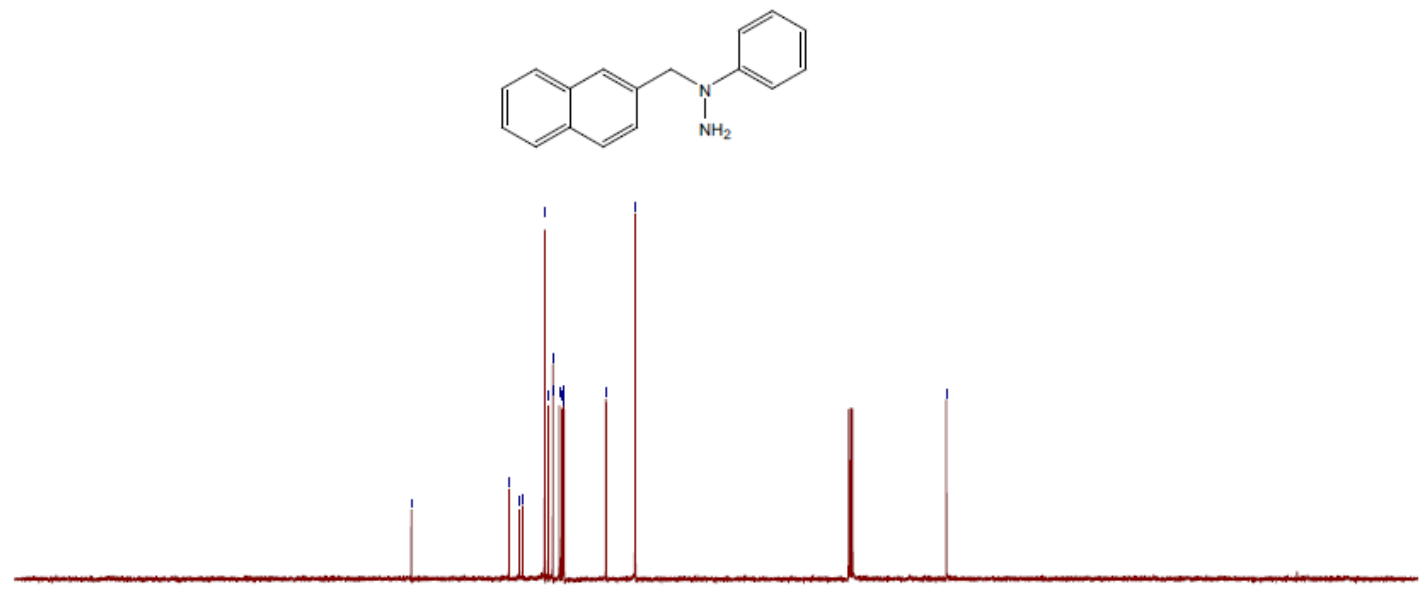

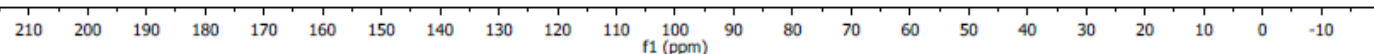


${ }^{1} \mathrm{H}$ NMR of $\mathbf{5 0}$

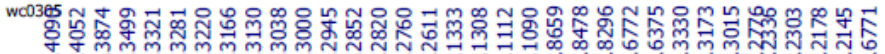

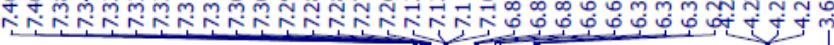
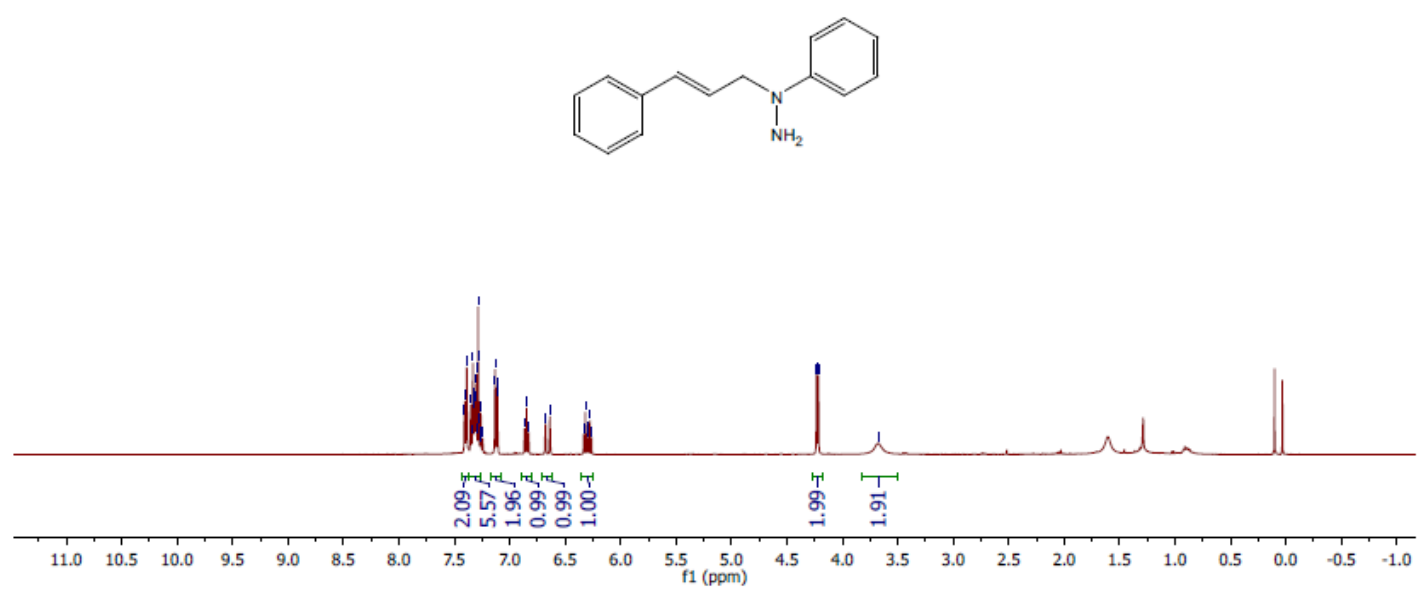

\section{${ }^{13}$ C NMR of 50}

wangc0305

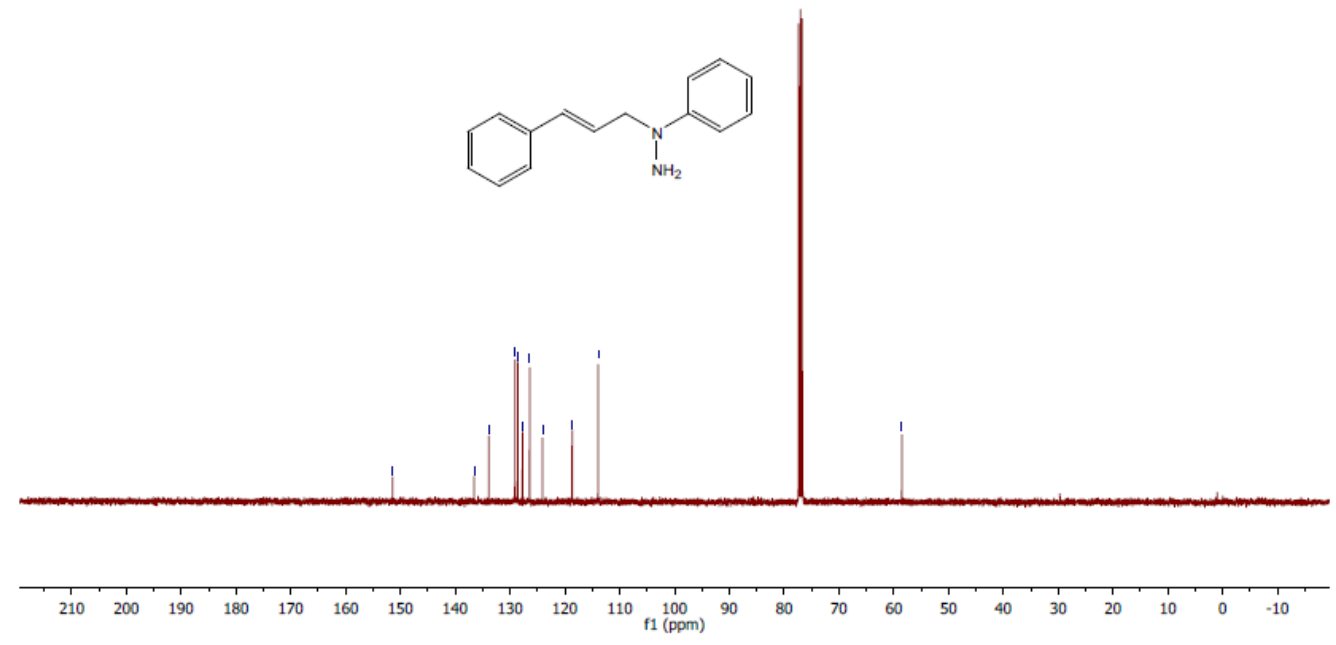


${ }^{1} \mathrm{H}$ NMR of $\mathbf{5 p}$

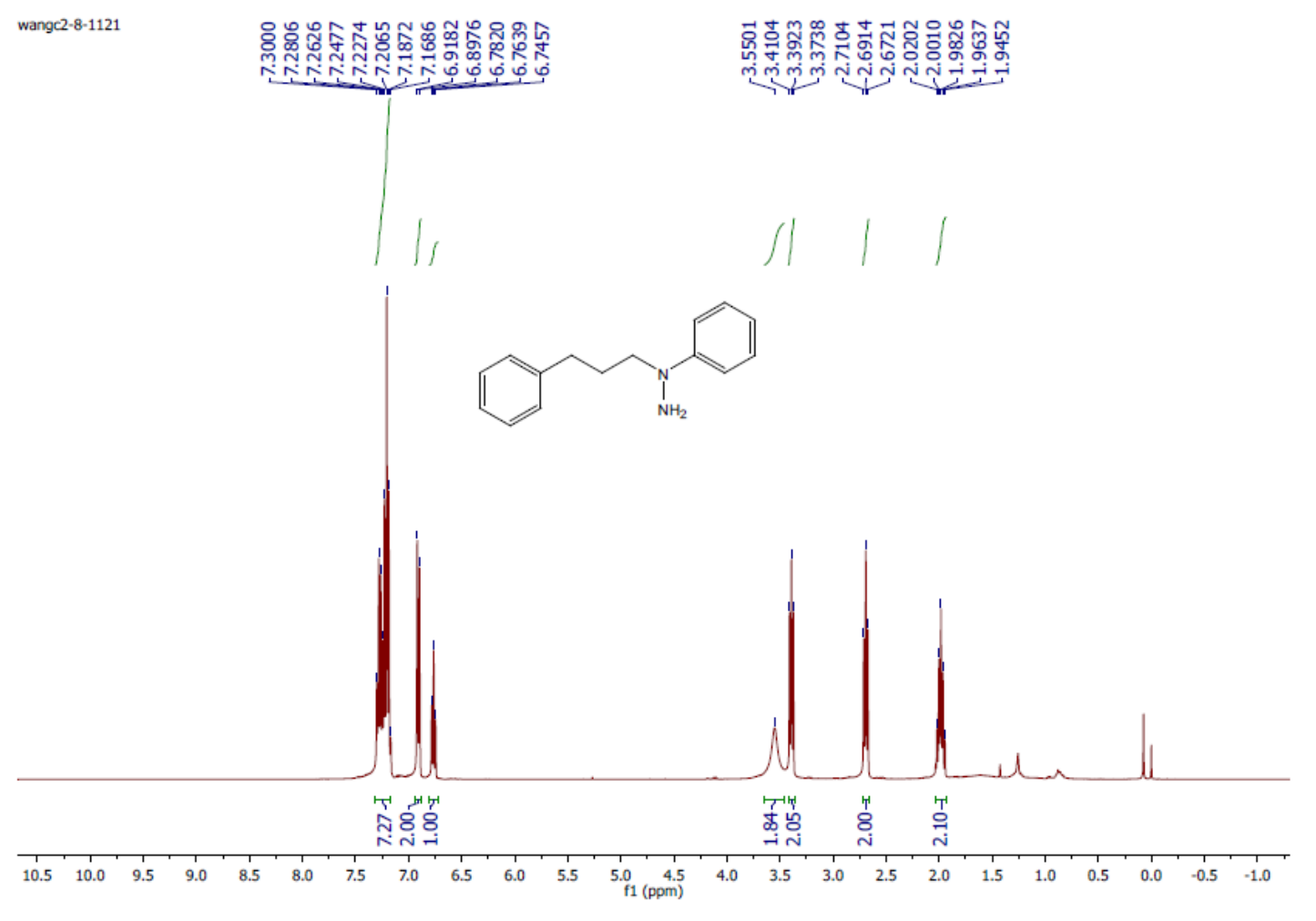

${ }^{13}$ C NMR of 5p

\begin{tabular}{|c|c|c|c|c|}
\hline wangc2-8-1121c & 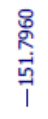 & 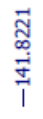 & 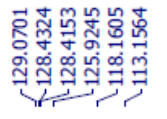 & 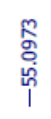 \\
\hline
\end{tabular}

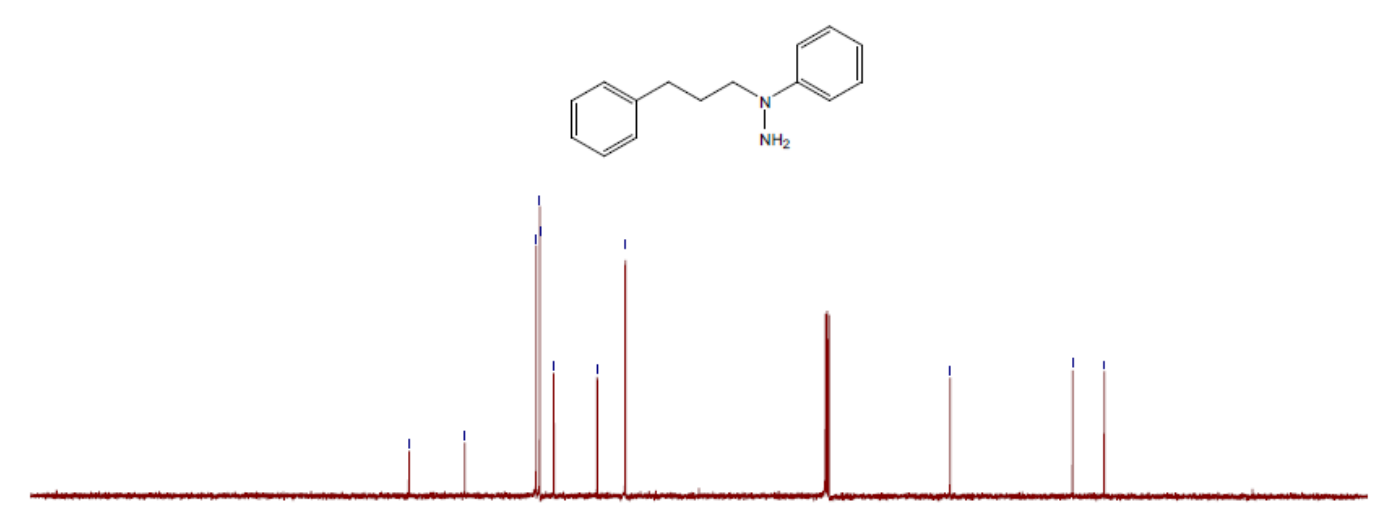

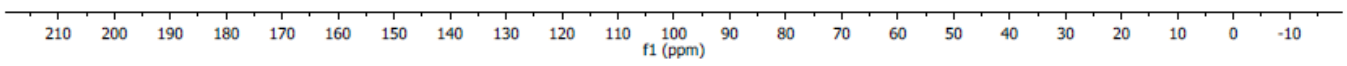




\section{${ }^{1} \mathrm{H}$ NMR of $\mathbf{5 q}$}

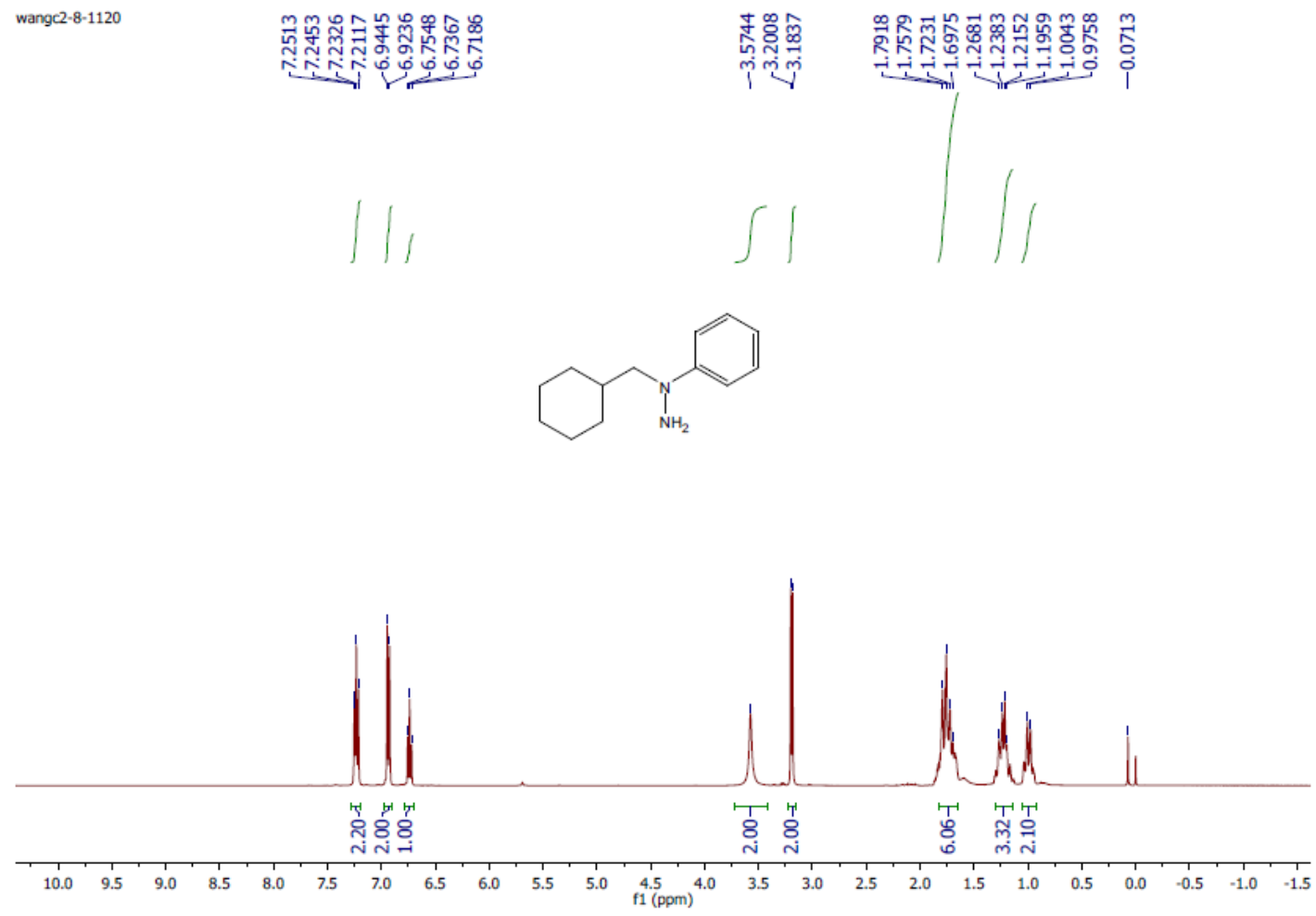

\section{${ }^{13}$ C NMR of $5 q$}

wangc2-8-1120 C

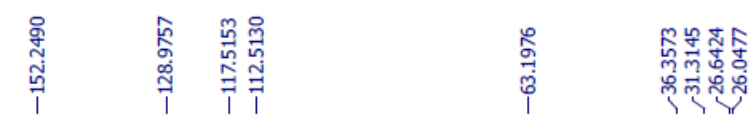<smiles>NC(CC1CCCCC1)c1ccccc1</smiles>

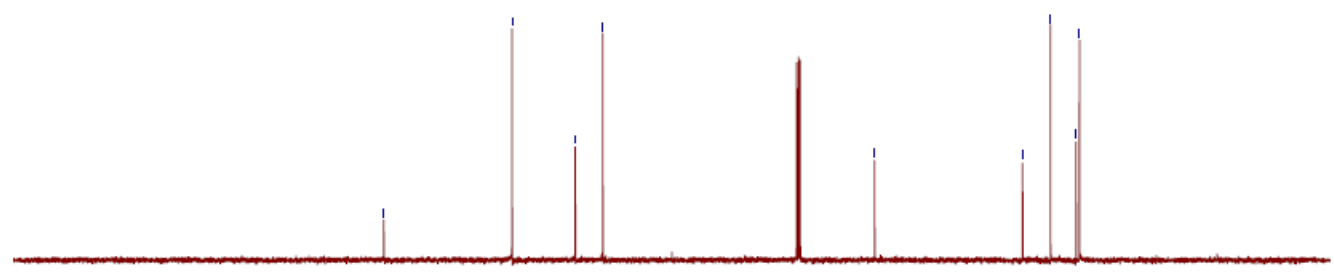

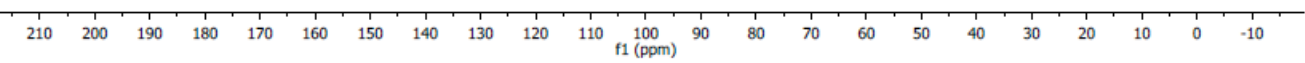


${ }^{1} \mathrm{H}$ NMR of $\mathbf{5 r}$

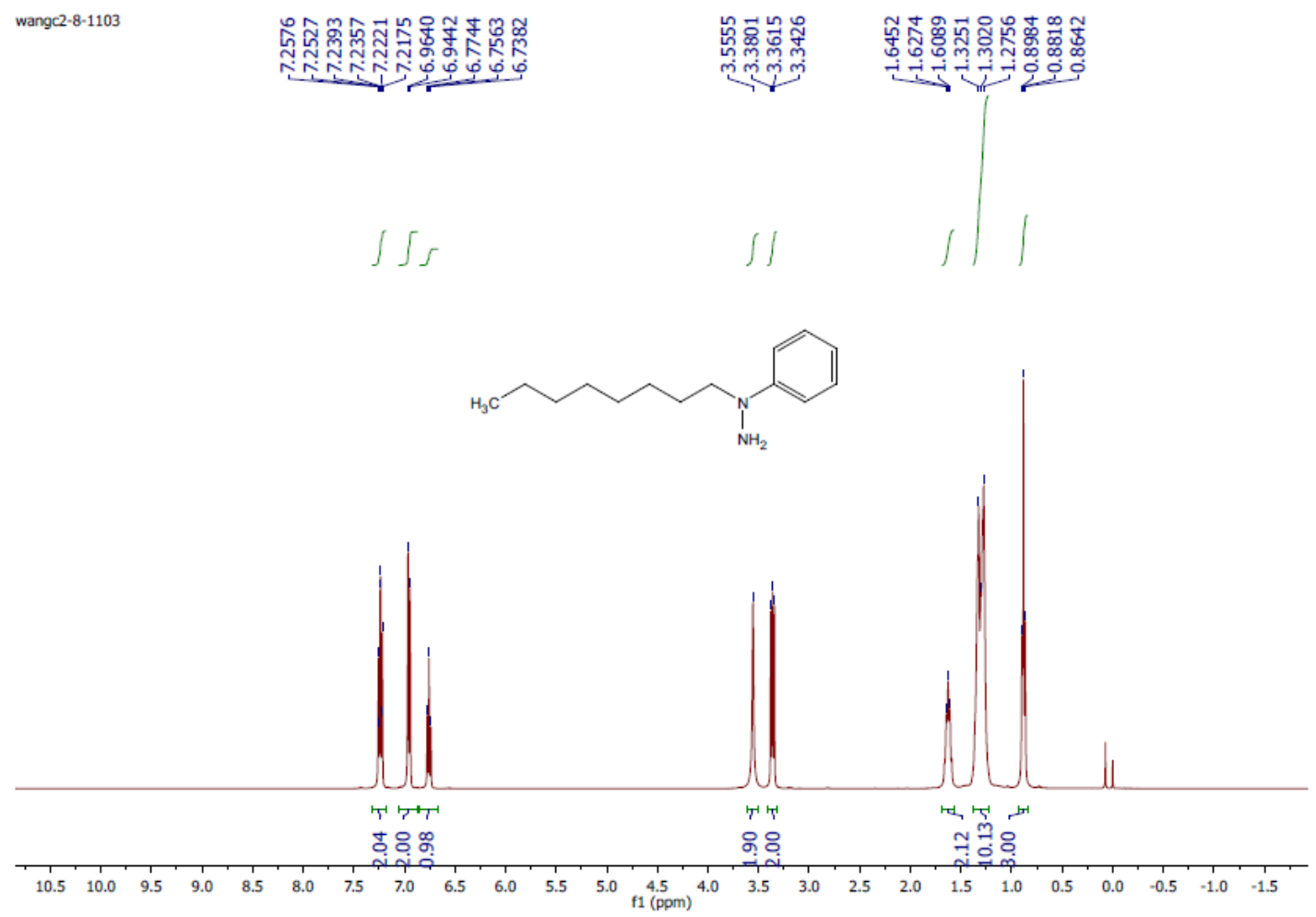

${ }^{13}$ C NMR of $5 r$

\begin{tabular}{|c|c|c|c|c|}
\hline wangc2-8-1103c & 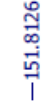 & $\begin{array}{l}\text { ఫิ } \\
\text { वे. } \\
\text { | }\end{array}$ & 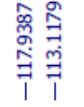 & 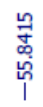 \\
\hline
\end{tabular}

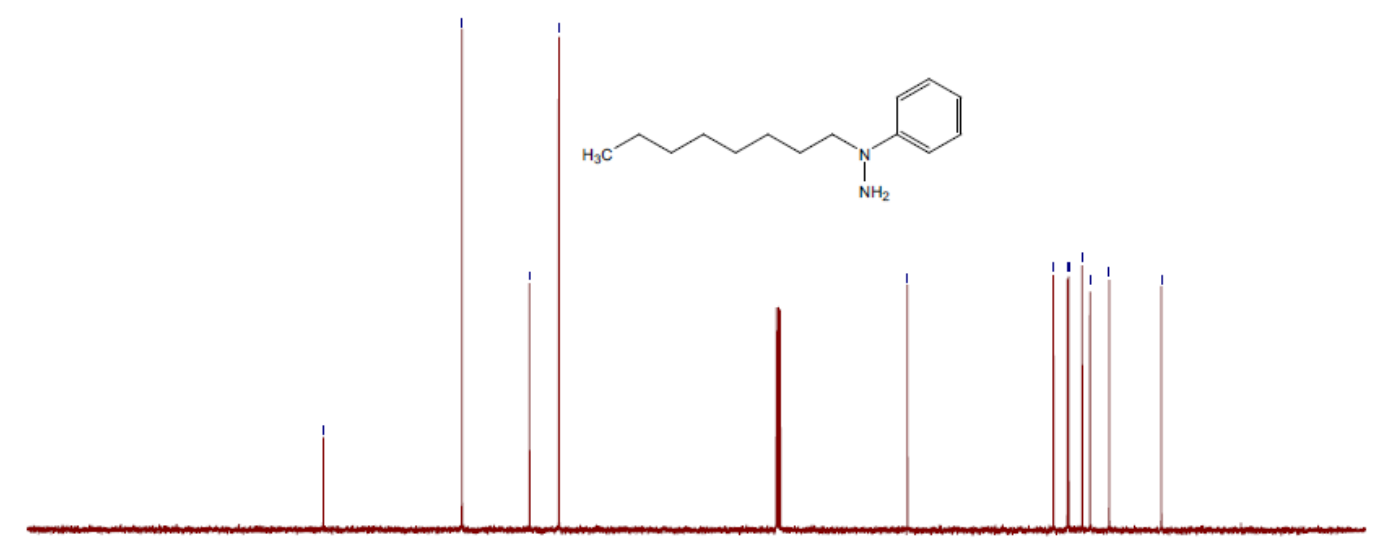

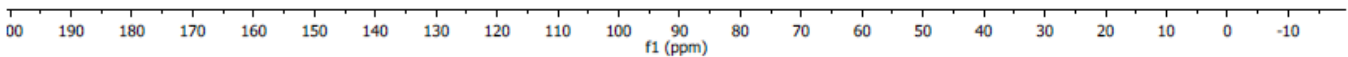


HPLC of 3a
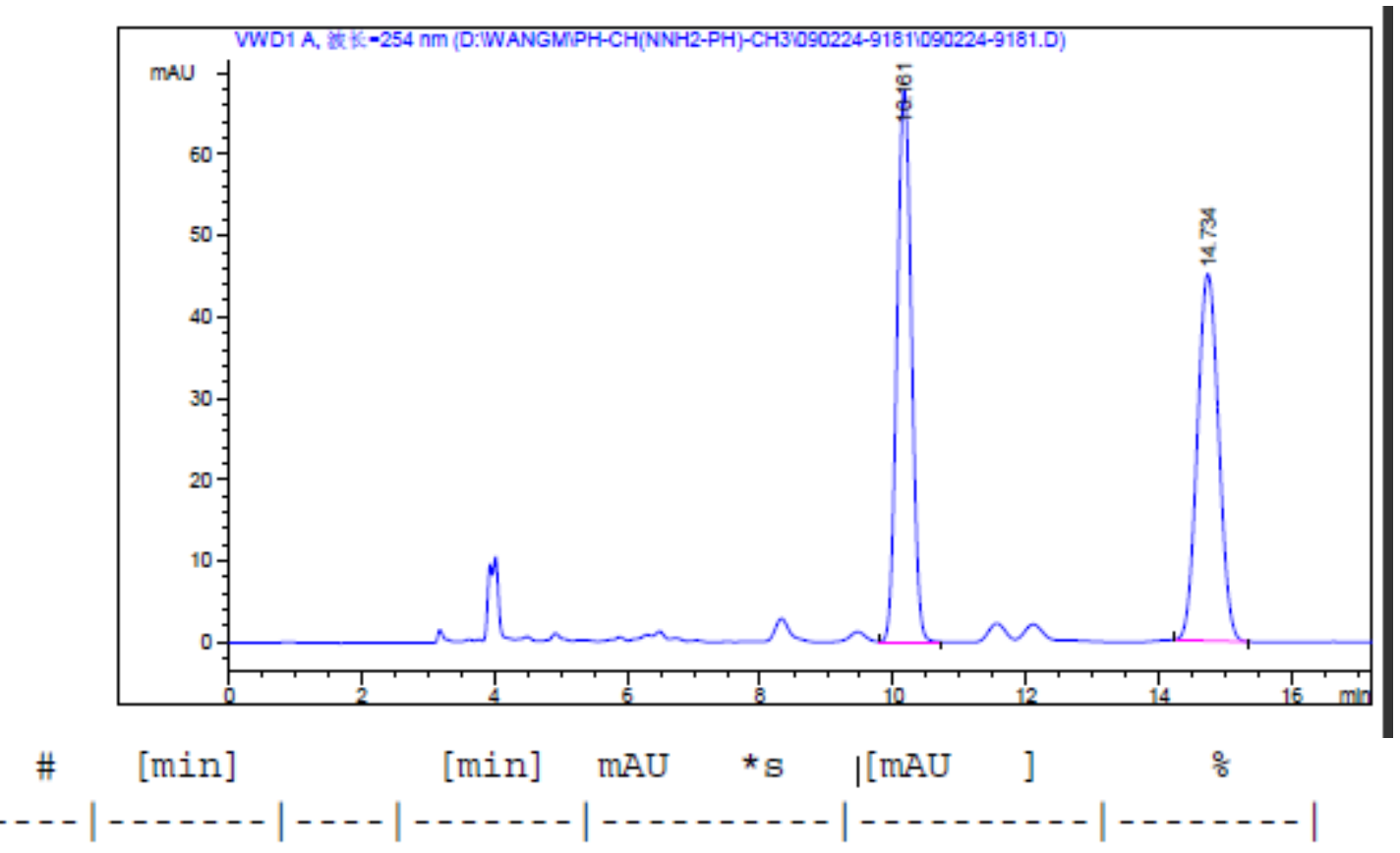

$\begin{array}{lllllll}1 & 10.161 & \mathrm{VB} & 0.2411 & 1031.04834 & 67.94851 & 50.5208\end{array}$

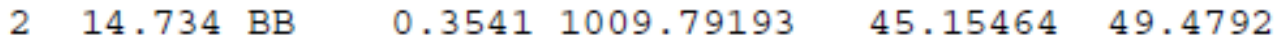

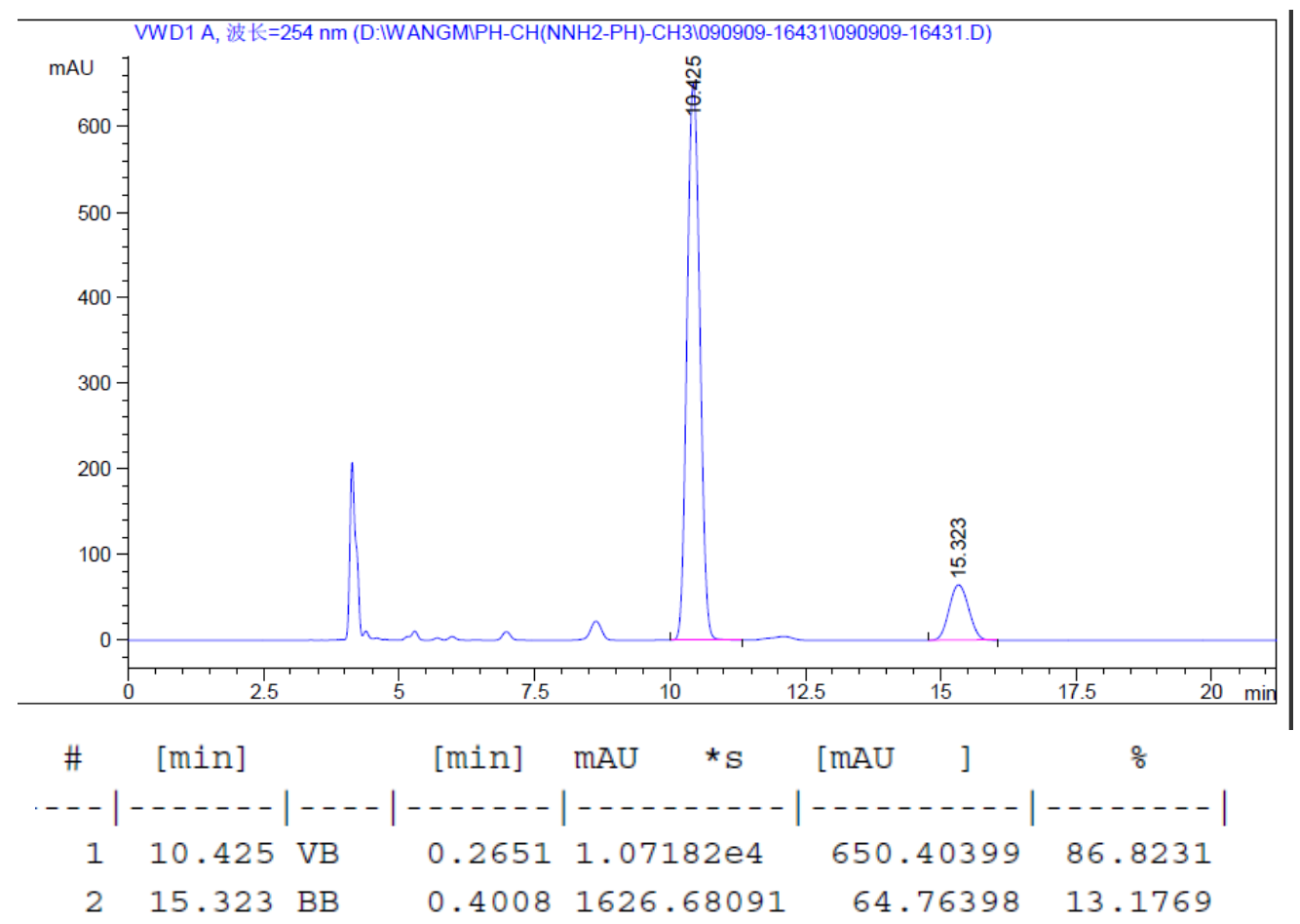

